\title{
On the Cauchy problem for noneffectively hyperbolic operators: The Gevrey 4 well-posedness
}

\author{
Enrico Bernardi and Tatsuo Nishitani
}

\begin{abstract}
For a hyperbolic second-order differential operator $P$, we study the relations between the maximal Gevrey index for the strong Gevrey well-posedness and some algebraic and geometric properties of the principal symbol $p$. If the Hamilton map $F_{p}$ of $p$ (the linearization of the Hamilton field $H_{p}$ along double characteristics) has nonzero real eigenvalues at every double characteristic (the so-called effectively hyperbolic case), then it is well known that the Cauchy problem for $P$ is well posed in any Gevrey class $1 \leq s<$ $+\infty$ for any lower-order term. In this paper we prove that if $p$ is noneffectively hyperbolic and, moreover, such that $\operatorname{Ker} F_{p}^{2} \cap \operatorname{Im} F_{p}^{2} \neq\{0\}$ on a $C^{\infty}$ double characteristic manifold $\Sigma$ of codimension 3, assuming that there is no null bicharacteristic landing $\Sigma$ tangentially, then the Cauchy problem for $P$ is well posed in the Gevrey class $1 \leq s<4$ for any lower-order term (strong Gevrey well-posedness with threshold 4), extending in particular via energy estimates a previous result of Hörmander in a model case.
\end{abstract}

\section{Introduction}

Let

$$
P(x, D)=-D_{0}^{2}+\sum_{|\alpha| \leq 2, \alpha_{0}<2} a_{\alpha}(x) D^{\alpha}=P_{2}+P_{1}+P_{0}
$$

be a second-order differential operator with real analytic or Gevrey $s$ class $a_{\alpha}(x)$ ( $s$ is close to 1 ) defined in an open neighborhood of the origin of $\mathbb{R}^{n+1}$ with the principal symbol $p(x, \xi)$, hyperbolic with respect to the $x_{0}$-direction, where $x=\left(x_{0}, x_{1}, \ldots, x_{n}\right)=\left(x_{0}, x^{\prime}\right), \xi=\left(\xi_{0}, \xi_{1}, \ldots, \xi_{n}\right)=\left(\xi_{0}, \xi^{\prime}\right)$. We are interested in the Cauchy problem for $P$ in the Gevrey classes when $p$ has double characteristics $\rho \in T^{*} \mathbb{R}^{n+1} \backslash\{0\}, p(\rho)=0, d p(\rho)=0$. We say that $f(x) \in \gamma^{(s)}\left(\mathbb{R}^{n}\right)$, the Gevrey class $s(\geq 1)$, if for any compact set $K \subset \mathbb{R}^{n}$, there exist $C>0, h>0$ such that

$$
\left|\partial_{x}^{\alpha} f(x)\right| \leq C h^{|\alpha|}|\alpha| !^{s}, \quad x \in K, \quad \forall \alpha \in \mathbb{N}^{n} .
$$

We set $\gamma_{0}^{(s)}\left(\mathbb{R}^{n}\right)=\gamma^{(s)}\left(\mathbb{R}^{n}\right) \cap C_{0}^{\infty}\left(\mathbb{R}^{n}\right)$.

Let $\rho$ be a double characteristic; then the Taylor expansion of $p$ around $\rho$ starts with a quadratic polynomial $p_{\rho}$ called the localization of $p$ at $\rho$, which is

Kyoto Journal of Mathematics, Vol. 51, No. 4 (2011), 767-810

DOI $10.1215 / 21562261-1424857$, (C) 2011 by Kyoto University

Received September 7, 2010. Revised January 24, 2011. Accepted February 2, 2011.

2010 Mathematics Subject Classification: Primary 35L15; Secondary 35B30. 
a hyperbolic polynomial (see [10]). The linearization of the Hamilton field $H_{p}$ at $\rho$ is called the Hamilton map $F_{p}(\rho)$ of $p$ at $\rho$ (see, e.g., [6], [10]). Note that $H_{p_{\rho}}(\theta)=F_{p} \theta$. If the Hamilton map has nonzero real eigenvalues at every double characteristic (effectively hyperbolic case), then the Cauchy problem for $P$ is well posed in $C^{\infty}$; in particular, in any Gevrey class $s, 1 \leq s<+\infty$ for any lowerorder term. To check this fact it is enough to apply the energy method developed in [13] to $e^{-x_{0}\left\langle D^{\prime}\right\rangle^{1 / s}} P e^{x_{0}\left\langle D^{\prime}\right\rangle^{1 / s}}(0<s \ll 1)$. In this paper we are thus interested in the optimal (maximal) Gevrey index $s^{*}$ such that the Cauchy problem for the noneffectively hyperbolic operator $P$ is well posed in the Gevrey class $s$ for $1 \leq s<s^{*}$ for any lower-order term and how this index $s^{*}$ relates to the geometry of the double characteristic manifold and null bicharacteristics.

As proved in [7], a hyperbolic quadratic form $Q$ in $\mathbb{R}^{2(n+1)}$ such that the Hamilton map $F_{Q}$ of $Q$ has no nonzero real eigenvalues can be written in a suitable system of symplectic coordinates according to the spectral structure of $F_{Q}$; according to whether $\operatorname{Ker} F_{Q}^{2} \cap \operatorname{Im} F_{Q}^{2}=\{0\}$ or $\operatorname{Ker} F_{Q}^{2} \cap \operatorname{Im} F_{Q}^{2} \neq\{0\}$, we have

$$
\begin{aligned}
& Q(x, \xi)=-\xi_{0}^{2}+\sum_{j=1}^{k} \mu_{j}\left(x_{j}^{2}+\xi_{j}^{2}\right)+\sum_{j=k+1}^{k+\ell} \xi_{j}^{2}, \\
& Q(x, \xi)=\left(-\xi_{0}^{2}+2 x_{1} \xi_{0}+\xi_{1}^{2}\right) / \sqrt{2}+\sum_{j=2}^{k} \mu_{j}\left(x_{j}^{2}+\xi_{j}^{2}\right)+\sum_{j=k+1}^{k+\ell} \xi_{j}^{2},
\end{aligned}
$$

respectively.

To symbols (1.2) or (1.3) there correspond (apart from harmonic oscillator contributions) the differential operators

$$
\begin{aligned}
& P_{n e, 1}=-D_{0}^{2}+\sum_{j=1}^{r} D_{j}^{2}, \quad r<n, \\
& P_{n e, 2}=-D_{0}^{2}+2 x_{1} D_{0} D_{n}+D_{1}^{2}+\sum_{j=2}^{r} D_{j}^{2}, \quad r<n,
\end{aligned}
$$

respectively. It is easy to see that the Cauchy problem for $P_{n e, 1}+S D_{n}$ is not well posed in the Gevrey class $s>2$ for $S \neq 0$, while it is well known that the Cauchy problem for $P$ is well posed in the Gevrey class $s, 1 \leq s<2$, for any lower-order term $P_{i}(i=0,1)$ which is a special case of a general result (see [4], [9]). On the other hand, in [7] an explicit formula of the forward fundamental solution of $P_{n e, 2}+S D_{n}$ is obtained for every $S \in \mathbb{C}$ which is a distribution on the Gevrey class 4 . Thus we see that the Cauchy problem for $P_{n e, 2}$ is well posed in the Gevrey class $1 \leq s<4$ for any $S \in \mathbb{C}$ and not well posed in the Gevrey class $s>4$ for $S \neq 0$ which follows from the explicit formula in [7] (for another proof of this fact, which is available for the more general case, see [16]). Therefore, in this paper, we consider the case (1.3) in a more general setting. 
In what follows we assume that $p$ vanishes exactly to order 2 on a $C^{\infty}$ manifold $\Sigma$, which means that near every $\rho \in \Sigma$ one can write

$$
p=-\xi_{0}^{2}+\sum_{j=1}^{r} \phi_{j}\left(x, \xi^{\prime}\right)^{2},
$$

where $d \phi_{j}$ are linearly independent at $\rho$ and $\Sigma$ is given near $\rho$ by

$$
\Sigma=\left\{(x, \xi) \mid \xi_{0}=0, \phi_{j}\left(x, \xi^{\prime}\right)=0, j=1, \ldots, r\right\}
$$

on which $F_{p}(\rho)$ has no nonzero real eigenvalues and

$$
\operatorname{Ker} F_{p}^{2}(\rho) \cap \operatorname{Im} F_{p}^{2}(\rho) \neq\{0\}, \quad \rho \in \Sigma .
$$

In this case the spectral properties of $F_{p}(\rho)$ are not enough by themselves to determine completely the behavior of null bicharacteristics near $\Sigma$ (see [15]), while the behavior of null bicharacteristic,

$$
\text { there is no null bicharacteristic falling on } \Sigma \text { tangentially, }
$$

is crucial to the $C^{\infty}$ well posedness (see [3]). In this paper we prove the following.

\section{THEOREM 1.1}

Assume (1.6) and (1.7). We also assume that the codimension of $\Sigma$ is 3. Then the Cauchy problem for $P$ is well posed in the Gevrey class $1 \leq s<4$ for any lower-order term; for any $f(x) \in \gamma^{(s)}\left(\mathbb{R}^{n+1}\right)$ vanishing for $x_{0} \leq 0$, there is $u(x)$ which is $C^{\infty}$, vanishing for $x_{0} \leq 0$ verifying

$$
P u=f
$$

near the origin.

The Gevrey index 4 is optimal in the following sense. Consider a model operator

$$
P_{\text {mod }}=-D_{0}^{2}+2 D_{0} D_{1}+x_{1}^{2} D_{n}^{2} \quad(n \geq 2)
$$

which verifies (1.6) and (1.7).

THEOREM 1.2 ([7, SECTION 9], [16, PROPOSITION 1.3])

The Cauchy problem for $P_{\mathrm{mod}}+S D_{n}$ with $S \neq 0$ is not locally solvable in any Gevrey class $s>4$.

This paper is organized as follows. In Section 2, we analyze our assumptions and we rewrite the principal symbol $p$ in a suitable form microlocally. In Section 3, giving heuristic arguments, we explain the idea of the proof of Theorem 1.1. In Section 4 we prepare symbol classes which are used in this paper and introduce a weight $\exp (\tilde{\phi})$ for an energy estimate which plays a crucial role to derive a priori estimates. In Sections 5 and 6 , we justify the heuristic arguments in Section 3. In Section 5, we prove required properties for the transformed operator $\exp (\tilde{\phi}) P \exp (-\tilde{\phi})$. In Section 6 we derive a priori estimates for the transformed operator, and using these a priori estimates we prove the existence of a parametrix 
of $P$ with finite propagation speed of wave front sets which proves Theorem 1.1. In Appendix A we collect several results about symbols without proofs which are used in this paper, and in Appendix B we present some formulas about $\exp (\tilde{\phi}) P \exp (-\tilde{\phi})$.

\section{Preliminaries}

Let $\bar{s}<4$; we prove Theorem 1.1 by proving the existence of a parametrix at any $\hat{\rho}^{\prime}=\left(0, \hat{x}^{\prime}, \hat{\xi}^{\prime}\right)$ of the Cauchy problem

$$
\begin{cases}P u=f, & f \in C^{0}\left([-T, T] ; \gamma^{(\bar{s})}\left(\mathbb{R}^{n}\right)\right), \\ u=0, & x_{0} \leq 0,\end{cases}
$$

with finite propagation speed of wave front sets, abbreviated as a parametrix with finite propagation speed in the following (see [11, Appendix]; here we define such parametrices by just requiring (A.3), (A.5) without (A.4)), where the support of $f$ is contained in $[0, T] \times\left\{\left|x^{\prime}\right| \leq K\right\}$ with some $K=K(f)>0$.

Let $\kappa$ be a local homogeneous canonical transformation $(y, \eta) \mapsto(x, \xi)$ from a neighborhood of $(\hat{y}, \hat{\eta})$ to a neighborhood of $(\hat{x}, \hat{\xi})$ such that $y_{0}=x_{0}$. Since $\kappa$ preserves the $y_{0}$-coordinate, a generating function of this canonical transformation has the form $x_{0} \eta_{0}+H\left(x, \eta^{\prime}\right)$. We assume that $H\left(x, \eta^{\prime}\right)=x^{\prime} \eta^{\prime}+\phi\left(x, \eta^{\prime}\right)$ with $\left|\nabla_{x, \eta^{\prime}} \phi\left(x, \eta^{\prime}\right)\right| \ll 1$ which is actually in our case (after a quadratic change of coordinates $x$ if necessary). Recall that

$$
\xi^{\prime}=\frac{\partial H\left(x, \eta^{\prime}\right)}{\partial x^{\prime}}, \quad y^{\prime}=\frac{\partial H\left(x, \eta^{\prime}\right)}{\partial \eta^{\prime}} .
$$

Let us denote by $S_{(s)}\left(m, g_{\rho, \delta}\right), 0 \leq \delta<\rho \leq 1$, the set of all smooth $a\left(x, \xi^{\prime}\right)$ verifying

$$
\left|\partial_{x}^{\beta} \partial_{\xi^{\prime}}^{\alpha} a\left(x, \xi^{\prime}\right)\right| \leq C A^{|\alpha+\beta|}|\alpha+\beta| !^{s} m\left\langle\xi^{\prime}\right\rangle^{m+\delta|\beta|-\rho|\alpha|}
$$

for all $\alpha, \beta$. Extending $\phi\left(x, \xi^{\prime}\right)$ so that it becomes homogeneous of degree 1 in $\xi^{\prime}$ and hence cutting off near $\xi^{\prime}=0$, which is in $S_{(s)}\left(\left\langle\xi^{\prime}\right\rangle, g_{1,0}\right)$, we consider the Fourier integral operators

$$
\begin{gathered}
\mathrm{Op}^{0}\left(e^{i \phi}\right) u=(2 \pi)^{-n} \int e^{i\left(x^{\prime}-y^{\prime}\right) \eta^{\prime}+i \phi\left(x, \eta^{\prime}\right)} u\left(y^{\prime}\right) d y^{\prime} d \eta^{\prime}, \\
\mathrm{Op}^{1}\left(e^{-i \phi}\right) u=(2 \pi)^{-n} \int e^{i\left(x^{\prime}-y^{\prime}\right) \eta^{\prime}-i \phi\left(x_{0}, y^{\prime}, \eta^{\prime}\right)} u\left(y^{\prime}\right) d y^{\prime} d \eta^{\prime},
\end{gathered}
$$

where $x_{0}$ is regarded as a parameter. Let $0<\rho \leq 1$; then we have the following.

PROPOSITION 2.1

Let $p \in S_{(s)}\left(\left\langle\xi^{\prime}\right\rangle^{m}, g_{1,0}\right)$ and $\phi \in S_{(s)}\left(\left\langle\xi^{\prime}\right\rangle, g_{1,0}\right)$ be real valued. Then we have

$$
\mathrm{Op}^{0}\left(e^{i \phi}\right) \mathrm{Op}^{0}(p) \mathrm{Op}^{1}\left(e^{-i \phi}\right)=\mathrm{Op}^{0}(\tilde{p})+\mathrm{Op}^{0}(r),
$$

where $\tilde{p} \in S_{(s)}\left(\left\langle\xi^{\prime}\right\rangle^{m}, g_{1,0}\right)$ and $r \in S_{((1+2 \rho) s+\epsilon)}\left(e^{-c\left\langle\xi^{\prime}\right\rangle^{1 / s}}, g_{\rho, 0}\right)$ for any $\epsilon>0$ with some $c>0$. Here

$$
\tilde{p}\left(x, \xi^{\prime}\right)=J\left(x, \xi^{\prime}\right) p\left(x_{0}, \nabla_{\eta^{\prime}} H\left(x, \Xi^{\prime}\right), \Xi^{\prime}\right)+S_{(s)}\left(\left\langle\xi^{\prime}\right\rangle^{m-1}, g_{1,0}\right),
$$


where $\Xi^{\prime}\left(x, \xi^{\prime}\right)$ verifies $\nabla_{x^{\prime}} H\left(x, \Xi^{\prime}\right)=\xi^{\prime}, H\left(x, \eta^{\prime}\right)=x^{\prime} \eta^{\prime}+\phi\left(x, \eta^{\prime}\right)$, and

$$
J\left(x, \xi^{\prime}\right)=\operatorname{det}\left[\frac{\partial \Xi^{\prime}\left(x, \xi^{\prime}\right)}{\partial \xi^{\prime}}\right] \text {. }
$$

Proof

The proof is standard (see e.g., [5], [12]) except for the Gevrey estimates for $\tilde{p}$ and $r$. We only sketch how to get the Gevrey estimates for $r$. Let us write $\mathrm{Op}^{0}\left(e^{i \phi}\right) \mathrm{Op}^{0}(p)=\mathrm{Op}^{0}\left(b_{1}\right)+\mathrm{Op}^{0}\left(b_{2}\right)$, where

$$
b_{i}=(2 \pi)^{-2} \int e^{-i y^{\prime} \eta^{\prime}+i \phi\left(x, \xi^{\prime}+\eta^{\prime}\right)} \chi_{i}\left(\xi^{\prime}, \eta^{\prime}\right) p\left(x^{\prime}+y^{\prime}, \xi^{\prime}\right) d y^{\prime} d \eta^{\prime}
$$

with $\chi_{1}=\chi\left(\eta^{\prime}\left\langle\xi^{\prime}\right\rangle^{-1}\right), \chi_{2}=1-\chi_{1}$. Here $\chi\left(x^{\prime}\right) \in \gamma_{0}^{(s)}\left(\mathbb{R}^{n}\right)$ is such that $\chi\left(x^{\prime}\right)=1$ for $\left|x^{\prime}\right| \leq 1 / 4$ and $\chi\left(x^{\prime}\right)=0$ for $\left|x^{\prime}\right| \geq 1 / 2$. We see easily that $b_{1}=e^{i \phi} q$ with $q \in S_{(s)}\left(\left\langle\xi^{\prime}\right\rangle^{m}, g_{1,0}\right)$. We examine $b_{2}$. Let $s_{1}=s+\epsilon>s$. From Corollary A.3 we have

$$
\begin{aligned}
& \left|\partial_{x^{\prime}}^{\beta} \partial_{\xi^{\prime}}^{\alpha} e^{i \phi\left(x, \xi^{\prime}+\eta^{\prime}\right)}\left\langle D_{y^{\prime}}\right\rangle^{N}\left\langle\eta^{\prime}\right\rangle^{-N} \chi_{2} p\left(x^{\prime}+y^{\prime}, \xi^{\prime}\right)\right| \\
& \quad \leq C A^{|\alpha+\beta|+N}|\alpha+\beta| !^{s_{1}} e^{c\left\langle\eta^{\prime}\right\rangle^{1 / s_{1}}} N !^{s}\left\langle\eta^{\prime}\right\rangle^{-N} \\
& \quad \leq C A^{|\alpha+\beta|+N}|\alpha+\beta| !^{s_{1}} e^{c\left\langle\eta^{\prime}\right\rangle^{1 / s_{1}}}\left\langle\xi^{\prime}\right\rangle^{-\rho|\alpha|} N !^{s}\left\langle\eta^{\prime}\right\rangle^{-N+\rho|\alpha|} .
\end{aligned}
$$

We choose $N=[\rho|\alpha|+\ell]$ such that the right-hand side of (2.1) is bounded by

$$
C A^{|\alpha+\beta|}(|\alpha+\beta| !)^{s_{1}+\rho s}\left\langle\xi^{\prime}\right\rangle^{-\rho|\alpha|} e^{c\left\langle\eta^{\prime}\right\rangle^{1 / s_{1}}}\left(A \ell^{s} /\left\langle\eta^{\prime}\right\rangle\right)^{\ell} .
$$

Choosing $\ell$ such that $\ell=\left[\left(A^{-1} e^{-1}\left\langle\eta^{\prime}\right\rangle\right)^{1 / s}\right]$ and noting $s_{1}>s$, we conclude that

$$
b_{2} \in S_{\left(s_{1}+\rho s\right)}\left(e^{-c\left\langle\xi^{\prime}\right\rangle^{1 / s}}, g_{\rho, 0}\right) .
$$

By standard arguments and the same type estimates as (2.1), we see

$$
\mathrm{Op}^{0}\left(e^{i \phi} q\right) \mathrm{Op}^{0}\left(e^{-i \phi}\right)=\mathrm{Op}^{0}(\tilde{p})+\mathrm{Op}^{0}(\tilde{r})
$$

where $\tilde{p} \in S_{(s)}\left(\left\langle\xi^{\prime}\right\rangle^{m}, g_{1,0}\right)$ verifies the assertion of Proposition 2.1 and $\tilde{r} \in$ $S_{((1+\rho) s+\epsilon)}\left(e^{-c\left\langle\xi^{\prime}\right\rangle^{1 / s}}, g_{\rho, 0}\right)$. Repeating arguments similar to (2.1), we get

$$
\mathrm{Op}^{0}\left(b_{2}\right) \mathrm{Op}^{0}\left(e^{-i \phi}\right)=\mathrm{Op}^{0}(c)
$$

with $c \in S_{\left(s_{1}+2 \rho s\right)}\left(e^{-c\left\langle\xi^{\prime}\right\rangle^{1 / s}}, g_{\rho, 0}\right)$, which proves the assertion.

For any $k \in \mathbb{N}$ there is $j \in S_{(s)}\left(1, g_{1,0}\right)$ such that $\operatorname{Op}^{0}(J) \mathrm{Op}^{0}(j)-1 \in$ $\mathrm{Op}^{0}\left(S_{(s)}\left(\left\langle\xi^{\prime}\right\rangle^{-k}, g_{1,0}\right)\right)$, and hence we conclude that

$$
\begin{aligned}
& \mathrm{Op}^{0}\left(e^{i \phi}\right) P \mathrm{Op}^{1}\left(e^{-i \phi}\right) \mathrm{Op}^{0}(j) \\
& =\operatorname{Op}^{0}\left(p\left(\kappa^{-1}(x, \xi)\right)\right) \\
& \quad+\operatorname{Op}^{0}\left(S_{(s)}\left(\left\langle\xi^{\prime}\right\rangle^{-k}, g_{1,0}\right) \xi_{0}^{2}+\sum_{j=0}^{1} S_{(s)}\left(\left\langle\xi^{\prime}\right\rangle^{1-j}, g_{1,0}\right) \xi_{0}^{j}\right) \\
& \quad+\mathrm{Op}^{0}\left(\sum_{j=0}^{2} S_{((1+3 \rho) s+\epsilon)}\left(e^{-c\left\langle\xi^{\prime}\right\rangle^{1 / s}}, g_{\rho, 0}\right) \xi_{0}^{j}\right)
\end{aligned}
$$


where $\kappa:\left(x_{0}, x^{\prime}+\nabla_{\eta^{\prime}} \phi\left(x, \eta^{\prime}\right), \eta_{0}, \eta^{\prime}\right) \mapsto\left(x_{0}, x^{\prime}, \eta_{0}+\partial_{x_{0}} \phi, \eta^{\prime}+\nabla_{x^{\prime}} \phi\left(x, \eta^{\prime}\right)\right)$.

Let us fix any $\rho=\left(0, x^{\prime}, 0, \xi^{\prime}\right) \in \Sigma$; we work in a conic neighborhood of $\rho^{\prime}=$ $\left(0, x^{\prime}, \xi^{\prime}\right)$. Since the codimension of $\Sigma$ is 3 , one can write

$$
p=-\xi_{0}^{2}+\phi_{1}^{2}+\phi_{2}^{2} \text {. }
$$

We set $\phi_{0}=\xi_{0}$. Consider $\left\{\phi_{0}, \phi_{j}\right\}(\rho), j=1,2$, and suppose $\left\{\phi_{0}, \phi_{j}\right\}(\rho)=0$, $j=1,2$. With $q=\phi_{1}^{2}+\phi_{2}^{2}$ we would have $\operatorname{Ker} F_{p}^{2} \cap \operatorname{Im} F_{p}^{2}=\operatorname{Ker} F_{q}^{2} \cap \operatorname{Im} F_{q}^{2}$ which contradicts (1.6) since $\operatorname{Ker} F_{q}^{2} \cap \operatorname{Im} F_{q}^{2}=\{0\}$ for $q$ is nonnegative. Thus considering $\left(\tilde{\phi}_{j}\right)_{j=1,2}=O\left(\phi_{j}\right)_{j=1,2}$ with a suitable smooth orthogonal $O$, we may assume that $\left\{\phi_{0}, \phi_{1}\right\}(\rho) \neq 0$ and $\left\{\phi_{0}, \phi_{2}\right\}$ is a linear combination of $\phi_{1}$ and $\phi_{2}$. We next examine $\left\{\phi_{1}, \phi_{2}\right\}(\rho) \neq 0$. If $\left\{\phi_{1}, \phi_{2}\right\}(\rho)=0$, then $p$ would be effectively hyperbolic at $\rho$. Indeed, $H_{\phi_{1}} \in(T \Sigma)^{\sigma}$ and

$$
p_{\rho}\left(H_{\phi_{1}}\right)=-\sigma\left(H_{\phi_{0}}, H_{\phi_{1}}\right)^{2}+\sigma\left(H_{\phi_{2}}, H_{\phi_{1}}\right)^{2}=-\sigma\left(H_{\phi_{0}}, H_{\phi_{1}}\right)^{2}<0 ;
$$

hence it follows from [6, Corollary 1.4.7] that $p$ is effectively hyperbolic at $\rho$.

\section{LEMMA 2.1}

One can write $p$ as

$$
\begin{gathered}
p=-\left(\xi_{0}+\phi_{1}\right)\left(\xi_{0}-\phi_{1}\right)+\phi_{2}^{2}, \\
\left\{\xi_{0}+\phi_{1}, \phi_{j}\right\}=0, j=1,2,\left\{\phi_{1}, \phi_{2}\right\} \neq 0 \quad \text { on } \Sigma .
\end{gathered}
$$

Proof

Recall that

$$
p=-\xi_{0}^{2}+\phi_{1}^{2}+\phi_{2}^{2} .
$$

Let $0 \neq X=a H_{\phi_{0}}+b H_{\phi_{1}}+c H_{\phi_{2}} \in \operatorname{Im} F_{p}^{2} \cap \operatorname{Ker} F_{p}^{2}$, which exists by our assumption. Since $X=F_{p}\left(\alpha H_{\phi_{0}}+\beta H_{\phi_{1}}+\gamma H_{\phi_{2}}\right)$, it follows that

$$
a=-2 \beta\left\{\phi_{0}, \phi_{1}\right\}, \quad c=2 \beta\left\{\phi_{2}, \phi_{1}\right\} .
$$

From $F_{p}^{2}(X)=0$ we see that

$$
b\left[\left\{\phi_{0}, \phi_{1}\right\}^{2}-\left\{\phi_{1}, \phi_{2}\right\}^{2}\right]=0, \quad \beta\left[\left\{\phi_{0}, \phi_{1}\right\}^{2}-\left\{\phi_{1}, \phi_{2}\right\}^{2}\right]=0 .
$$

If $\left\{\phi_{0}, \phi_{1}\right\}^{2}-\left\{\phi_{1}, \phi_{2}\right\}^{2} \neq 0$, then we would have $X=0$, which is a contradiction. Thus we have proved

$$
\left\{\xi_{0}, \phi_{1}\right\}^{2}=\left\{\phi_{1}, \phi_{2}\right\}^{2} \quad \text { on } \Sigma .
$$

We may assume that $\left\{\xi_{0}, \phi_{1}\right\}=\left\{\phi_{1}, \phi_{2}\right\}$ so that $\left\{\xi_{0}+\phi_{2}, \phi_{1}\right\}=0$ on $\Sigma$. Writing

$$
p=-\left(\xi_{0}+\phi_{2}\right)\left(\xi_{0}-\phi_{2}\right)+\phi_{1}^{2}
$$

and exchanging $\phi_{1}$ and $\phi_{2}$, we get the desired assertion.

Since $\left\{\xi_{0}+\phi_{1}, \phi_{2}\right\}=0$ and $\left\{\phi_{1}, \phi_{2}\right\} \neq 0$, it follows that $\left\{\xi_{0}, \phi_{2}\right\} \neq 0$. Hence one can write $\phi_{2}=a\left(x, \xi^{\prime}\right)\left(x_{0}-\psi\left(x, \xi^{\prime}\right)\right)$, where $a\left(x, \xi^{\prime}\right)$ is nonvanishing and $\psi$ is independent of $x_{0}$. Since $\left\{\phi_{1}, \phi_{2}\right\} \neq 0$ and hence $d \psi \neq 0$, one can take a new 
system of homogeneous symplectic coordinates $(y, \eta)$ so that

$$
y_{0}=x_{0}, \quad \eta_{0}=\xi_{0}, \quad y_{1}=\psi\left(x, \xi^{\prime}\right) .
$$

Hence we may assume, for instance, that $\phi_{2}=a\left(x, \xi^{\prime}\right)\left(x_{0}-x_{1}\right)$. Make a linear change of coordinates

$$
y_{0}=x_{0}, \quad y_{1}=x_{1}-x_{0} .
$$

We get

$$
\left\{\begin{array}{l}
p=-\left(\xi_{0}+\xi_{1}+\phi_{1}\right)\left(\xi_{0}+\xi_{1}-\phi_{1}\right)+\phi_{2}^{2}, \\
\phi_{2}=a\left(x, \xi^{\prime}\right) x_{1}, \quad a\left(x, \xi^{\prime}\right) \neq 0, \\
\left\{\xi_{0}+\xi_{1}+\phi_{1}, \phi_{j}\right\}=0, \quad j=1,2,\left\{\phi_{1}, \phi_{2}\right\} \neq 0 \text { on } \Sigma .
\end{array}\right.
$$

Here we recall a result that characterizes when (1.7) occurs. Choose a smooth vector field $z(\rho)$ on $\Sigma$ such that $z(\rho) \in \operatorname{Ker} F_{p}^{2}(\rho) \cap \operatorname{Im} F_{p}^{2}(\rho)$ and $F_{p}(\rho) z(\rho) \neq 0$. Then we have the following.

PROPOSITION 2.2 ([2, THEOREM 2.1], [14, THEOREM 4.1])

Let $S(x, \xi)$ be a smooth real-valued function vanishing on $\Sigma$ such that $H_{S}(\rho)$ is proportional to $z(\rho)$ modulo $\operatorname{Ker} F_{p}(\rho)$. Then there is no null bicharacteristic falling on $\Sigma$ if and only if $H_{S}^{3} p=0$ on $\Sigma$.

Let us write with $\Lambda=\xi_{0}+\xi_{1}+\phi_{1}$,

$$
p=-\Lambda^{2}+2 \phi_{1} \Lambda+\phi_{2}^{2}, \quad\left\{\Lambda, \phi_{2}\right\}=\alpha \phi_{1}+\beta \phi_{2} .
$$

\section{LEMMA 2.2}

Assume (1.7). Then we have $\alpha=0$ on $\Sigma$. In particular, we have

$$
\left\{\Lambda, \phi_{2}\right\}=a \phi_{1}^{2}+b \phi_{2} \text {. }
$$

\section{Proof}

Note that $F_{p} H_{\phi_{1}}=2\left\{\phi_{2}, \phi_{1}\right\} H_{\phi_{2}}$ and hence $H_{\phi_{2}} \in \operatorname{Im} F_{p}^{2}$. On the other hand, since $F_{p} H_{\phi_{2}}=\left\{\phi_{1}, \phi_{2}\right\} H_{\Lambda}$, hence $H_{\phi_{2}} \in \operatorname{Ker} F_{p}^{2}$ because $F_{p} H_{\Lambda}=0$. Thus we may take $S=\phi_{2}$ in Proposition 2.2. Then $H_{S}^{3} p=0$ implies that

$$
\left\{\phi_{2},\left\{\phi_{2}, \Lambda\right\}\right\}=0,
$$

which proves $\alpha=0$ on $\Sigma$ and hence the result.

Since the existence of a parametrix with finite propagation speed (i.e., a parametrix verifying (A.3), (A.5) in [11]) is invariant under conjugation with Fourier integral operator associated to a local homogeneous canonical transformation preserving the $x_{0}$-coordinate (see the proof of [11, Proposition A.5]), we can assume that the operator we are studying has the form in the right-hand side in (2.2), and in particular, we can assume that (2.3) and (2.4) hold near the reference double point $\rho$.

We extend $p$ globally outside the reference double point. 


\section{LEMMA 2.3}

Assume that (2.3) and (2.4) are satisfied in a conic neighborhood of $\rho^{\prime}=\left(0, x^{\prime}, \xi^{\prime}\right)$. Then we can extend $\phi_{1}\left(x, \xi^{\prime}\right)$ such that $\phi_{1}\left(x, \xi^{\prime}\right) \in S\left(\left\langle\xi^{\prime}\right\rangle, g_{1,0}\right)$,

$$
\phi_{2}\left(x, \xi^{\prime}\right)=a\left(x, \xi^{\prime}\right) \psi\left(x_{1}\right),
$$

where $a \in S\left(1, g_{1,0}\right)$ with $C^{-1} \leq a\left(x, \xi^{\prime}\right) \leq C$ and $\psi\left(x_{1}\right)$ is nondecreasing, $\left|\psi\left(x_{1}\right)\right| \leq 1$ and $\psi\left(x_{1}\right)=x_{1}$ near $x_{1}=0$, and

$$
\left\{\phi_{1}, \psi\right\} \geq c>0
$$

provided $\left|\psi\left(x_{1}\right)\right|+\left\langle\xi^{\prime}\right\rangle^{-2}\left|\phi_{1}\left(x, \xi^{\prime}\right)\right|^{2}$ is small. Moreover, there exists $c_{i}\left(x, \xi^{\prime}\right) \in$ $S\left(\left\langle\xi^{\prime}\right\rangle^{i}, g_{1,0}\right)$ which vanishes in a conic neighborhood of $\rho^{\prime}$ such that

$$
\left\{\begin{array}{c}
\left\{\Lambda+c_{1} \psi+c_{0} \phi_{1}+c_{-1} \phi_{1}^{2}, \phi_{1}\right\} \\
\quad=d_{0} \phi_{1}+d_{0}^{\prime} \phi_{2}+d_{1} \sqrt{\psi^{2}+\left\langle\xi^{\prime}\right\rangle^{-4} \phi_{1}^{4}}, \\
\left\{\Lambda+c_{1} \psi+c_{0} \phi_{1}+c_{-1} \phi_{1}^{2}, \psi\right\} \\
\quad=d_{-2} \phi_{1}^{2}+d_{-1} \phi_{2}+d_{0}^{\prime \prime} \sqrt{\psi^{2}+\left\langle\xi^{\prime}\right\rangle^{-4} \phi_{1}^{4}}
\end{array}\right.
$$

with some $d_{i}, d_{i}^{\prime}, d_{i}^{\prime \prime} \in S\left(\left\langle\xi^{\prime}\right\rangle^{i}, g_{1,0}\right)$, where $\Lambda=\xi_{0}+\xi_{1}+\phi_{1}$.

Proof

We may assume that $\left\{\phi_{1}, \phi_{2}\right\}\left(\rho^{\prime}\right)>0$ and hence $\partial_{\xi_{1}} \phi_{1}\left(\rho^{\prime}\right)>0$. Thus one can write $\phi_{1}=b\left(x, \xi^{\prime}\right)\left(\xi_{1}-\psi_{1}\left(x, \xi^{\prime}\right)\right)$ near $\rho^{\prime}$, where $\psi_{1}$ is independent of $\xi_{1}$. Extending $b$ and $\psi_{1}$ so that $b \in S\left(1, g_{1,0}\right), C^{-1} \leq b \leq C$ and $\psi_{1} \in S\left(\left\langle\xi^{\prime}\right\rangle, g_{1,0}\right)$, the assertion (2.5) follows immediately because $x_{1}-\psi=0$ near $x_{1}=0$. We turn to (2.6). From (2.4) it follows that $\{\Lambda, \psi\}=d_{-2} \phi_{1}^{2}+d_{-1} \phi_{2}+R$ with $R \in S\left(1, g_{1,0}\right)$ which vanishes in a neighborhood of $\rho^{\prime}$. Note that

$$
\left\{\phi_{1}, \psi\right\}+K \sqrt{\psi^{2}+\left\langle\xi^{\prime}\right\rangle-4} \phi_{1}^{4} \geq c>0
$$

with a large $K>0$ thanks to (2.5). Hence we can write

$$
R=a\left(\left\{\phi_{1}, \psi\right\}+r\right), \quad r=K \sqrt{\psi^{2}+\left\langle\xi^{\prime}\right\rangle^{-4} \phi_{1}^{4}},
$$

with $a \in S\left(1, g_{1,0}\right)$ vanishing in a neighborhood of $\rho^{\prime}$. Thus we have

$$
\begin{aligned}
& \left\{\Lambda+c_{0} \phi_{1}+c_{-1} \phi_{1}^{2}, \psi\right\}=\left(d_{-2}+\left\{c_{-1}, \psi\right\}\right) \phi_{1}^{2}+d_{-1} \phi_{2} \\
& +\left(a+c_{0}\right)\left\{\phi_{1}, \psi\right\}+a r+\left(\left\{c_{0}, \psi\right\}+2 c_{-1}\left\{\phi_{1}, \psi\right\}\right) \phi_{1} .
\end{aligned}
$$

Choose $c_{0}=-a$ and $2 c_{-1}=\{a, \psi\}\left(\left\{\phi_{1}, \psi\right\}+r\right)^{-1}$ so that $c_{i} \in S\left(\left\langle\xi^{\prime}\right\rangle^{i}, g_{1,0}\right)$ vanishes in a neighborhood of $\rho^{\prime}$; we get

$$
\left\{\Lambda+c_{0} \phi_{1}+c_{-1} \phi_{1}^{2}, \psi\right\}=d_{-2} \phi_{1}^{2}+d_{-1} \phi_{2}+d_{0} r .
$$

A similar argument proves that there is $c_{1}$-vanishing in a neighborhood of $\rho^{\prime}$ such that $\left\{\Lambda+c_{1} \psi, \phi_{1}\right\}=d_{0} \phi_{1}+d_{0}^{\prime} \phi_{2}+d_{1} r$. These prove the assertion (2.6).

Replacing $\Lambda$ by $\Lambda+c_{1} \psi+c_{0} \phi+c_{-1} \phi_{1}^{2}$, the resulting symbol $-\Lambda^{2}+2 \phi_{1} \Lambda+\phi_{2}^{2}$ differs from the original one by

$$
C_{0} \xi_{0}+C_{1}
$$


where $C_{i} \in S\left(\left\langle\xi^{\prime}\right\rangle^{i}, g_{1,0}\right)$ vanishes in a neighborhood of $\rho^{\prime}$. Thus it sufffices to show the existence of a parametrix with finite propagation speed for the operator where $\Lambda$ is replaced by $\Lambda+c_{1} \psi+c_{0} \phi+c_{-1} \phi_{1}^{2}$ (see the proof of [11, Lemma A.1].

\section{Idea of the proof of Theorem 1.1}

We prove Theorem 1.1 by deriving a priori estimates for the transformed operator. In this section we give a heuristic argument on how to do it, in which symbols and operators are not strictly distinguished. Moreover, we write $p \sim q$ if the main parts of $p$ and $q$ coincide, and write $p \preceq q$ if the main part of $q-p$ is nonnegative.

The symbol of the operator that we are studying looks like (replacing $\xi_{0}+$ $\xi_{1}+\phi_{1}$ by $\left.\xi_{0}\right)$

$$
P=-\xi_{0}^{2}+2 \phi_{1}\langle\xi\rangle \xi_{0}+\phi_{2}^{2}\langle\xi\rangle^{2},
$$

where $\phi_{j}$ are homogeneous of degree 0 , and verifies

$$
\left\{\xi_{0}, \phi_{2}\right\}=a \phi_{1}^{2}+b \phi_{2}, \quad\left\{\xi_{0}, \phi_{1}\right\}=a^{\prime} \phi_{1}+b^{\prime} \phi_{2}, \quad\langle\xi\rangle\left\{\phi_{1}, \phi_{2}\right\} \geq c(>0) .
$$

With $w=\sqrt{\phi_{1}^{4}+\langle\xi\rangle^{-1}}$ we introduce a weight function

$$
\Phi=i\langle\xi\rangle^{1 / 4}\left\{\log \left(\phi_{2}+i w\right)-\log \left(\phi_{2}-i w\right)\right\}=-2\langle\xi\rangle^{1 / 4} \arg \left(\phi_{2}+i w\right) .
$$

Conjugate $e^{\gamma\langle D\rangle^{1 / 4} x_{0}}$ to $P$; then $e^{-\gamma\langle D\rangle^{1 / 4} x_{0}} P e^{\gamma\langle D\rangle^{1 / 4} x_{0}}$ yields

$$
P \sim-\left(\xi_{0}-i \gamma\langle\xi\rangle^{1 / 4}\right)^{2}+2 \phi_{1}\langle\xi\rangle\left(\xi_{0}-i \gamma\langle\xi\rangle^{1 / 4}\right)+\phi_{2}^{2}\langle\xi\rangle^{2} .
$$

We rewrite this in the form

$$
\begin{aligned}
P \sim & -\left(\xi_{0}-i \gamma\langle\xi\rangle^{1 / 4}+k \phi_{1}^{3}\langle\xi\rangle\right)\left(\xi_{0}-i \gamma\langle\xi\rangle^{1 / 4}-k \phi_{1}^{3}\langle\xi\rangle\right) \\
& +2 \phi_{1}\langle\xi\rangle\left(\xi_{0}-i \gamma\langle\xi\rangle^{1 / 4}-k \phi_{1}^{3}\langle\xi\rangle\right) \\
& +2 k \phi_{1}^{4}\langle\xi\rangle^{2}+\phi_{2}^{2}\langle\xi\rangle^{2}-k^{2} \phi_{1}^{6}\langle\xi\rangle^{2} \\
= & -M \Lambda+2 \phi_{1}\langle\xi\rangle \Lambda+Q
\end{aligned}
$$

with a positive constant $k>0$. We next conjugate $e^{\Phi}$ with $P$; that is, we study

$$
e^{\Phi} P e^{-\Phi} \sim-e^{\Phi} \Lambda e^{-\Phi} \cdot e^{\Phi} M e^{-\Phi}+2 e^{\Phi} \phi_{1}\langle\xi\rangle e^{-\Phi} \cdot e^{\Phi} \Lambda e^{-\Phi}+e^{\Phi} Q e^{-\Phi} .
$$

Let $e^{\Phi} \Lambda e^{-\Phi}=\Lambda+\Lambda^{\prime}$; then the main part of $\Lambda^{\prime}$ consists of $e^{\Phi}\left\{\xi_{0}, e^{-\Phi}\right\} / i=$ $i\left\{\xi_{0}, \Phi\right\}$ and $i e^{\Phi}\left\{\phi_{1}^{3}\langle\xi\rangle, e^{-\Phi}\right\}=-\left\{\phi_{1}^{3}\langle\xi\rangle, \Phi\right\}$. Note that

$$
i\left\{\xi_{0}, \Phi\right\}=-2\langle\xi\rangle^{1 / 4}\left\{\xi_{0}, \phi_{2}\right\} \frac{w}{\phi_{2}^{2}+w^{2}}+2\langle\xi\rangle^{1 / 4}\left\{\xi_{0}, w\right\} \frac{\phi_{2}}{\phi_{2}^{2}+w^{2}}
$$

and $\left\{\xi_{0}, w\right\}=2 w^{-1} \phi_{1}^{3}\left\{\xi_{0}, \phi_{1}\right\}$. By the assumption (3.1), we have

$$
\left|e^{\Phi}\left\{\xi_{0}, e^{-\Phi}\right\}\right| \leq C\langle\xi\rangle^{1 / 4} .
$$

We also note

$$
\left\{\phi_{1}^{3}\langle\xi\rangle, \Phi\right\} \sim 6\langle\xi\rangle^{1 / 4} \phi_{1}^{2}\langle\xi\rangle\left\{\phi_{1}, \phi_{2}\right\} \frac{w}{\phi_{2}^{2}+w^{2}}
$$

which shows that

$$
\left|e^{\Phi}\left\{k \phi_{1}^{3}\langle\xi\rangle, e^{-\Phi}\right\}\right| \leq C k\langle\xi\rangle^{1 / 4} .
$$


We now study $e^{\Phi} \phi_{1}\langle D\rangle e^{-\Phi}=\phi_{1}\langle D\rangle+\tilde{\phi}_{1}$. The main part of $\tilde{\phi}_{1}$ is

$$
\frac{1}{i} e^{\Phi}\left\{\phi_{1}\langle\xi\rangle, e^{-\Phi}\right\} \sim 2 i\langle\xi\rangle^{5 / 4}\left\{\phi_{1}, \phi_{2}\right\} \frac{w}{\phi_{2}^{2}+w^{2}},
$$

and hence

$$
\operatorname{Im}\left(\frac{1}{i} e^{\Phi}\left\{\phi_{1}\langle\xi\rangle, e^{-\Phi}\right\}\right) \geq c\langle\xi\rangle^{1 / 4} \frac{w}{\phi_{2}^{2}+w^{2}}, \quad c>0
$$

by assumption (3.1), which gives a positive contribution, crucial to the control of not only lower-order terms but also of other terms caused by conjugation of $e^{\Phi}$.

We consider $e^{\Phi} Q e^{-\Phi}=Q+i Q^{\prime}$. The main part of $Q^{\prime}$ is

$$
\begin{array}{r}
-e^{\Phi}\left\{\phi_{2}^{2}\langle\xi\rangle^{2}+k \phi_{1}^{4}\langle\xi\rangle^{2}, e^{-\Phi}\right\}=\left\{\phi_{2}^{2}\langle\xi\rangle^{2}+k \phi_{1}^{4}\langle\xi\rangle^{2}, \Phi\right\} \\
\sim-8\langle\xi\rangle^{2+1 / 4}\left\{\phi_{2}, \phi_{1}\right\}\left[\frac{\phi_{2}^{2} \phi_{1}^{3}}{\left(\phi_{2}^{2}+w^{2}\right) w}+k \frac{\phi_{1}^{3} w}{\phi_{2}^{2}+w^{2}}\right]
\end{array}
$$

which gives

$$
\left|Q^{\prime}\right| \preceq\langle\xi\rangle^{5 / 4} w^{1 / 2}
$$

because $\left|\phi_{1}\right| \leq w^{1 / 2}$. Now $e^{\Phi} P e^{-\Phi}$ looks as follows:

$$
\tilde{P}=-M \Lambda+2 B \Lambda+(Q+\langle D\rangle)-\langle D\rangle,
$$

where we regard $Q+\langle D\rangle$ as a new $Q$ and $-\langle D\rangle$ as a lower-order term. Note that

$$
\begin{array}{r}
\Lambda \sim \xi_{0}-i \gamma\langle\xi\rangle^{1 / 4}-k \phi_{1}^{3}\langle\xi\rangle+i \tilde{\lambda}=\xi_{0}-i \gamma\langle\xi\rangle^{1 / 4}+\lambda, \quad|\operatorname{Im} \lambda| \leq C\langle\xi\rangle^{1 / 4}, \\
M \sim \xi_{0}-i \gamma\langle\xi\rangle^{1 / 4}+k \phi_{1}^{3}\langle\xi\rangle+i \tilde{m}=\xi_{0}-i \gamma\langle\xi\rangle^{1 / 4}+m, \quad|\operatorname{Im} m| \leq C\langle\xi\rangle^{1 / 4}, \\
B \sim \phi_{1}\langle\xi\rangle+2 i\langle\xi\rangle^{5 / 4}\left\{\phi_{1}, \phi_{2}\right\} \frac{w}{\phi_{2}^{2}+w^{2}}, \quad \operatorname{Im} B \geq c\langle\xi\rangle^{1 / 4} \frac{w}{\phi_{2}^{2}+w^{2}}, \\
Q+\langle D\rangle \sim \phi_{2}^{2}\langle\xi\rangle^{2}+k \phi_{1}^{4}\langle\xi\rangle^{2}+\langle\xi\rangle+i Q^{\prime}, \quad\left|Q^{\prime}\right| \preceq\langle\xi\rangle^{5 / 4} w^{1 / 2} .
\end{array}
$$

We recall an energy identity (see Proposition 6.1)

$$
\begin{aligned}
2 \operatorname{Im}(\tilde{P} u, \Lambda u)= & \frac{d}{d x_{0}}\left(\|\Lambda u\|^{2}+((\operatorname{Re} Q) u, u)\right) \\
& +2 \gamma\left\|\langle D\rangle^{1 / 8} \Lambda u\right\|^{2}+2 \gamma \operatorname{Re}\left(\langle D\rangle^{1 / 4}(\operatorname{Re} Q) u, u\right) \\
& +2((\operatorname{Im} B) \Lambda u, \Lambda u)+2((\operatorname{Im} m) \Lambda u, \Lambda u)+2 \operatorname{Re}(\Lambda u,(\operatorname{Im} Q) u) \\
& +\operatorname{Im}\left(\left[D_{0}-\operatorname{Re} \lambda, \operatorname{Re} Q\right] u, u\right)+2 \operatorname{Re}((\operatorname{Re} Q) u,(\operatorname{Im} \lambda) u) .
\end{aligned}
$$

The terms $2((\operatorname{Im} m) \Lambda u, \Lambda u)$ and $2 \operatorname{Re}((\operatorname{Re} Q) u,(\operatorname{Im} \lambda) u)$ are easily estimated because $|\operatorname{Im} \lambda|,|\operatorname{Im} m| \leq C\langle\xi\rangle^{1 / 4}$. In what follows we note that the terms

$$
\epsilon\left|\left(\frac{\langle\xi\rangle^{1 / 4} w}{\phi_{2}^{2}+w^{2}} \Lambda u, \Lambda u\right)\right|, \quad K\left|\left(\langle\xi\rangle^{1 / 4}\left(\phi_{2}^{2}\langle\xi\rangle^{2}+\phi_{1}^{4}\langle\xi\rangle^{2}+\langle\xi\rangle\right) u, u\right)\right|
$$

with small $\epsilon>0$ and any $K>0$ can be controlled by $2((\operatorname{Im} B) \Lambda u, \Lambda u)$ and $2 \gamma \operatorname{Re}\left(\langle D\rangle^{1 / 4}(\operatorname{Re} Q+\langle D\rangle) u, u\right)$, taking $\gamma$ large if necessary. 
To estimate $(\operatorname{Im} Q u, \Lambda u)$ it suffices to note that

$$
\begin{aligned}
\left|\left(\langle\xi\rangle^{5 / 4} w^{1 / 2} u, \Lambda u\right)\right| \leq & \epsilon\left|\left(\frac{\langle\xi\rangle^{1 / 4} w}{\phi_{2}^{2}+w^{2}} \Lambda u, \Lambda u\right)\right| \\
& +\epsilon^{-1}\left|\left(\langle\xi\rangle^{2+1 / 4}\left(\phi_{2}^{2}+w^{2}\right) u, u\right)\right| \\
\leq & \epsilon\left|\left(\frac{\langle\xi\rangle^{1 / 4} w}{\phi_{2}^{2}+w^{2}} \Lambda u, \Lambda u\right)\right| \\
& +\epsilon^{-1}\left|\left(\langle\xi\rangle^{1 / 4}\left(\phi_{2}^{2}\langle\xi\rangle^{2}+\phi_{1}^{4}\langle\xi\rangle^{2}+\langle\xi\rangle\right) u, u\right)\right|
\end{aligned}
$$

To see how any lower-order term can be controlled, it is enough to note that

$$
\begin{aligned}
& 2 K|(\langle\xi\rangle u, \Lambda u)| \\
& \quad \leq \epsilon^{-1} K^{2}\left|\left(\frac{\left(\phi_{2}^{2}+w^{2}\right)\langle\xi\rangle^{2}}{\langle\xi\rangle^{1 / 4} w} u, u\right)\right|+\epsilon\left|\left(\frac{\langle\xi\rangle^{1 / 4} w}{\phi_{2}^{2}+w^{2}} \Lambda u, \Lambda u\right)\right| \\
& \leq \epsilon^{-1} K^{2}\left|\left(\langle\xi\rangle^{2+1 / 4}\left(\phi_{2}^{2}+w^{2}\right) u, u\right)\right|+\epsilon\left|\left(\frac{\langle\xi\rangle^{1 / 4} w}{\phi_{2}^{2}+w^{2}} \Lambda u, \Lambda u\right)\right| \\
& \quad \leq \epsilon^{-1} K^{2}\left|\left(\langle\xi\rangle^{1 / 4}\left(\phi_{2}^{2}\langle\xi\rangle^{2}+\phi_{1}^{4}\langle\xi\rangle^{2}+\langle\xi\rangle\right) u, u\right)\right|+\epsilon\left|\left(\frac{\langle\xi\rangle^{1 / 4} w}{\phi_{2}^{2}+w^{2}} \Lambda u, \Lambda u\right)\right|
\end{aligned}
$$

because $\left(\langle\xi\rangle^{1 / 4} w\right)^{-1} \leq\langle\xi\rangle^{1 / 4}$. We finally check the commutator term, $\left[D_{0}-\operatorname{Re} \lambda\right.$, $\operatorname{Re} Q]$. Note that

$$
\begin{aligned}
& {\left[D_{0}-\operatorname{Re} \lambda, \operatorname{Re} Q\right] \sim-i\left\{\xi_{0}-k \phi_{1}^{3}\langle\xi\rangle, \phi_{2}^{2}\langle\xi\rangle^{2}+k \phi_{1}^{4}\langle\xi\rangle^{2}\right\}} \\
& \quad \sim-2 i \phi_{2}\langle\xi\rangle^{2}\left\{\xi_{0}, \phi_{2}\right\}-4 i k\langle\xi\rangle^{2} \phi_{1}^{3}\left\{\xi_{0}, \phi_{1}\right\}+6 i k\langle\xi\rangle^{3} \phi_{1}^{2} \phi_{2}\left\{\phi_{1}, \phi_{2}\right\}
\end{aligned}
$$

and hence

$$
\left|\operatorname{Im}\left[D_{0}-\operatorname{Re} \lambda, \operatorname{Re} Q\right]\right| \preceq C_{M}\left(\phi_{2}^{2}\langle\xi\rangle^{2}+\phi_{1}^{4}\langle\xi\rangle^{2}\right)
$$

because of (3.1). Thus we conclude

$$
\begin{aligned}
& 2 \operatorname{Im}((\tilde{P}+K\langle D\rangle) u, \Lambda u) \succeq \frac{d}{d x_{0}}\left(\|\Lambda u\|^{2}+((\operatorname{Re} Q) u, u)\right) \\
& +c \gamma\left\|\langle D\rangle^{1 / 8} \Lambda u\right\|^{2}+c \gamma \operatorname{Re}\left(\langle D\rangle^{1 / 4}(\operatorname{Re} Q) u, u\right),
\end{aligned}
$$

and hence an a priori estimate is obtained. We justify these heuristic arguments in Sections 4-6.

\section{Symbols}

In this section we precisely define our weight function and the symbols with which we work. As observed in the end of Section 2, we can assume that $P(x, \xi)$ is globally defined and the principal symbol $p(x, \xi)$,

$$
p(x, \xi)=-\Lambda^{2}+2 \phi_{2} \Lambda+\phi_{2}^{2}, \quad \Lambda=\xi_{0}+\lambda_{1},
$$


verifies the conditions (2.5) and

$$
\left\{\begin{array}{l}
\left\{\Lambda, \phi_{1}\right\}=d_{0} \phi_{1}+d_{0}^{\prime} \phi_{2}+d_{1} \sqrt{\psi^{2}+\left\langle\xi^{\prime}\right\rangle^{-4} \phi_{1}^{4}}, \\
\{\Lambda, \psi\}=d_{-2} \phi_{1}^{2}+d_{-1} \phi_{2}+d_{0}^{\prime \prime} \sqrt{\psi^{2}+\left\langle\xi^{\prime}\right\rangle^{-4} \phi_{1}^{4}}
\end{array}\right.
$$

We dilate the variable: $x_{0} \rightarrow \mu x_{0}$ (small $\mu>0$ ) so that we have

$$
\begin{aligned}
P(x, \xi, \mu) & =\mu^{2} P\left(\mu x_{0}, x^{\prime}, \mu^{-1} \xi_{0}, \xi^{\prime}\right) \\
& =p\left(\mu x_{0}, x^{\prime}, \xi_{0}, \mu \xi^{\prime}\right)+\mu P_{1}\left(\mu x_{0}, x^{\prime}, \xi_{0}, \mu \xi^{\prime}\right)+\mu^{2} P_{0}\left(\mu x_{0}, x^{\prime}, \xi_{0}, \mu \xi^{\prime}\right) \\
& =p(x, \xi, \mu)+P_{1}(x, \xi, \mu)+P_{0}(x, \xi, \mu) .
\end{aligned}
$$

In what follows we often write $p(x, \xi), \phi_{j}\left(x, \xi^{\prime}\right)$ for $p(x, \xi, \mu), \phi_{j}\left(x, \xi^{\prime}, \mu\right)$, dropping $\mu$.

Let us denote by $S_{(s)}(m, g)$ with

$$
g=\sum_{j=0}^{n} \delta_{j}^{2} d x_{j}^{2}+\sum_{j=1}^{n} \rho_{j}^{-2} d \xi_{j}^{2}
$$

the set of all smooth $a\left(x, \xi^{\prime} ; \mu\right)$ satisfying

$$
\left|\partial_{x}^{\beta} \partial_{\xi^{\prime}}^{\alpha} a\left(x, \xi^{\prime} ; \mu\right)\right| \leq C A^{|\alpha+\beta|}|\alpha+\beta| !^{s} m\left(x, \xi^{\prime} ; \mu\right) \delta^{\beta} \rho^{-\alpha}
$$

with some $C>0, A>0$ independent of $\mu$, where $\delta=\left(\delta_{1}\left(x, \xi^{\prime}, \mu\right), \ldots, \delta_{n}\left(x, \xi^{\prime}, \mu\right)\right)$, $\rho=\left(\rho_{1}\left(x, \xi^{\prime}, \mu\right), \ldots, \rho_{n}\left(x, \xi^{\prime}, \mu\right)\right)$, and $\delta_{j}, \delta_{j}$ are assumed to be in $S_{(s)}\left(\delta_{j}, g\right)$, $S_{(s)}\left(\rho_{j}, g\right)$, respectively.

We also denote by $S(m, g)$ the symbol class consisting of all smooth $a\left(x, \xi^{\prime}, \mu\right)$ verifying (4.2) with $C_{\alpha, \beta}$, independent of $\mu$, instead of $C A^{|\alpha+\beta|}|\alpha+\beta| !^{\text {s }}$. Note the following.

\section{LEMMA 4.1}

Let $a\left(x, \xi^{\prime}\right) \in S_{(s)}\left(\left\langle\xi^{\prime}\right\rangle^{k}, g_{1,0}\right)$. Then we have with, $g_{0}=\mu^{2} d x_{0}^{2}+\left|d x^{\prime}\right|^{2}+$ $\left\langle\xi^{\prime}\right\rangle_{\mu}^{-2}\left|d \xi^{\prime}\right|^{2},\left\langle\xi^{\prime}\right\rangle_{\mu}^{2}=\mu^{-2}+\left|\xi^{\prime}\right|^{2}=\mu^{-2}\left\langle\mu \xi^{\prime}\right\rangle^{2}$,

$$
a\left(\mu x_{0}, x^{\prime}, \mu \xi^{\prime}\right) \in S_{(s)}\left(\left\langle\mu \xi^{\prime}\right\rangle^{k}, g_{0}\right) .
$$

We rewrite $p(x, \xi)$ as

$$
\begin{aligned}
p= & -\left(\xi_{0}+\lambda_{1}+k\left\langle\mu \xi^{\prime}\right\rangle^{-2} \phi_{1}^{3}\right)\left(\xi_{0}+\lambda_{1}-k\left\langle\mu \xi^{\prime}\right\rangle^{-2} \phi_{1}^{3}\right) \\
& +2 \phi_{1}\left(\xi_{0}+\lambda_{1}-k\left\langle\mu \xi^{\prime}\right\rangle^{-2} \phi_{1}^{3}\right)+\phi_{2}^{2}+2 k\left\langle\mu \xi^{\prime}\right\rangle^{-2} \phi_{1}^{4}\left\{1-k / 2\left\langle\mu \xi^{\prime}\right\rangle^{-2} \phi_{1}^{2}\right\} .
\end{aligned}
$$

Taking a positive constant $k$ to be sufficiently small, we set

$$
Q=\phi_{2}^{2}+\theta^{2}, \quad \theta^{2}=2 k\left\langle\mu \xi^{\prime}\right\rangle^{-2} \phi_{1}^{4}\left\{1-k / 2\left\langle\mu \xi^{\prime}\right\rangle^{-2} \phi_{1}^{2}\right\},
$$

and note that $\theta\left(x, \xi^{\prime}\right) \in S\left(\left\langle\mu \xi^{\prime}\right\rangle, g_{0}\right)$ verifies $C^{-1}\left\langle\mu \xi^{\prime}\right\rangle^{-1} \phi_{1}^{2} \leq \theta \leq C\left\langle\mu \xi^{\prime}\right\rangle^{-1} \phi_{1}^{2}$ with some $C>0$. Thus one can write

$$
p=-M(x, \xi) \Lambda(x, \xi)+2 \phi_{1}\left(x, \xi^{\prime}\right) \Lambda(x, \xi)+Q\left(x, \xi^{\prime}\right),
$$


where $M=\xi_{0}+\lambda_{1}+k\left\langle\mu \xi^{\prime}\right\rangle^{-2} \phi_{1}^{3}, \Lambda=\xi_{0}+\lambda_{1}-k\left\langle\mu \xi^{\prime}\right\rangle^{-2} \phi_{1}^{3}$. Let us set

$$
w\left(x, \xi^{\prime}\right)=\sqrt{\left\langle\mu \xi^{\prime}\right\rangle^{-4} \phi_{1}\left(x, \xi^{\prime}\right)^{4}+\left\langle\xi^{\prime}\right\rangle_{\mu}^{-1}}
$$

then it follows from Corollary A.4 that

$$
\begin{aligned}
w & \in S_{(s)}\left(w, w^{-1}\left(\mu^{2} d x_{0}^{2}+\left|d x^{\prime}\right|^{2}+\left\langle\xi^{\prime}\right\rangle_{\mu}^{-2}\left|d \xi^{\prime}\right|^{2}\right)\right), \\
& \subset S_{(s)}\left(w,\left\langle\xi^{\prime}\right\rangle_{\mu}^{1 / 2}\left(\mu^{2} d x_{0}^{2}+\left|d x^{\prime}\right|^{2}+\left\langle\xi^{\prime}\right\rangle_{\mu}^{-2}\left|d \xi^{\prime}\right|^{2}\right)\right) .
\end{aligned}
$$

Let

$$
0<\kappa<\frac{1}{4}
$$

be fixed hereafter. Eventually we take $\kappa$ very close to $1 / 4$. We introduce the symbol

$$
\begin{aligned}
\phi & =i\left\langle\mu \xi^{\prime}\right\rangle^{\kappa}\left\{\log \left(\psi\left(x_{1}\right)+i w\left(x, \xi^{\prime}\right)\right)-\log \left(\psi\left(x_{1}\right)-i w\left(x, \xi^{\prime}\right)\right)\right\} \\
& =\left\langle\mu \xi^{\prime}\right\rangle^{\kappa} \arg \frac{\psi\left(x_{1}\right)-i w\left(x, \xi^{\prime}\right)}{\psi\left(x_{1}\right)+i w\left(x, \xi^{\prime}\right)}=-2\left\langle\mu \xi^{\prime}\right\rangle^{\kappa} \arg \left(\psi\left(x_{1}\right)+i w\right)
\end{aligned}
$$

and set

$$
r\left(x, \xi^{\prime}\right)=\sqrt{\psi\left(x_{1}\right)^{2}+w\left(x, \xi^{\prime}\right)^{2}}=\sqrt{\psi^{2}+\left\langle\mu \xi^{\prime}\right\rangle^{-4} \phi_{1}^{4}+\left\langle\xi^{\prime}\right\rangle_{\mu}^{-1}} .
$$

Then from Lemma A.6 it follows that

$$
\phi\left(x, \xi^{\prime}\right) \in S_{(s)}\left(\left\langle\mu \xi^{\prime}\right\rangle^{\kappa}, g\right), \quad r\left(x, \xi^{\prime}\right) \in S_{(s)}(r, g),
$$

where

$$
g=\left(r\left(x, \xi^{\prime}\right)^{-1}+w^{-1 / 2}\right)^{2} d x_{1}^{2}+w^{-1}\left(\mu^{2} d x_{0}^{2}+\left|d x^{\prime \prime}\right|^{2}\right)+w^{-1}\left\langle\xi^{\prime}\right\rangle_{\mu}^{-2}\left|d \xi^{\prime}\right|^{2}
$$

with $x^{\prime \prime}=\left(x_{2}, \ldots, x_{n}\right)$. We also use

$$
\begin{aligned}
g & \leq \hat{g}=\left\langle\xi^{\prime}\right\rangle_{\mu} d x_{1}^{2}+\left\langle\xi^{\prime}\right\rangle_{\mu}^{1 / 2}\left(\mu^{2} d x_{0}^{2}+\left|d x^{\prime \prime}\right|^{2}\right)+\left\langle\xi^{\prime}\right\rangle_{\mu}^{-3 / 2}\left|d \xi^{\prime}\right|^{2} \\
& \leq\left\langle\xi^{\prime}\right\rangle_{\mu}|d x|^{2}+\left\langle\xi^{\prime}\right\rangle_{\mu}^{-3 / 2}\left|d \xi^{\prime}\right|^{2}=\bar{g} .
\end{aligned}
$$

We now recall conditions (2.5) and (2.6) in terms of symbol classes.

LEMMA 4.2

We have

$$
\left\{\phi_{1}, \psi\right\} \geq c \mu
$$

provided $r\left(x, \xi^{\prime}\right)$ is small. Moreover, we have

$$
\left\{\begin{array}{l}
\{\Lambda, \psi\} \in \mu S(r, g), \quad\left\{\Lambda, \phi_{1}\right\} \in \mu S\left(\left(r+w^{1 / 2}\right)\left\langle\mu \xi^{\prime}\right\rangle, g\right), \\
\partial_{\xi_{1}} \Lambda \in \mu S(r, g), \quad \partial_{\xi_{1}}^{2} \Lambda \in \mu S\left(\left(r+w^{1 / 2}\right)\left\langle\xi^{\prime}\right\rangle_{\mu}^{-1}, g\right) .
\end{array}\right.
$$

\section{Proof}

The first two assertions follow from (2.5) and (4.1) immediately. Note that

$$
\{\Lambda, \psi\}=C_{-2} \phi_{1}^{2}+C_{-1} \phi_{2}+C_{0} r
$$


with some $C_{j} \in \mu S_{(s)}\left(\left\langle\mu \xi^{\prime}\right\rangle^{j}, g_{0}\right)$. Noting that $\left\{\Lambda, x_{1}\right\}=\left\{\Lambda, x_{1}-\psi\right\}+\{\Lambda, \psi\}$ and $\left\{\Lambda, x_{1}-\psi\right\}$ vanishes if $x_{1}$ is small, one can then write

$$
\partial_{\xi_{1}} \Lambda=\{\Lambda, \psi\}+C \psi\left(x_{1}\right)
$$

with $C \in \mu S(1, g)$, which shows $\partial_{\xi_{1}} \Lambda \in \mu S_{(s)}(r, g)$. From this expression it is clear that $\partial_{\xi_{1}}^{2} \Lambda \in \mu S_{(s)}\left(\left(r+w^{1 / 2}\right)\left\langle\xi^{\prime}\right\rangle_{\mu}^{-1}, g\right)$.

\section{Transformed symbols}

Take $\kappa^{\prime}$ so that

$$
\kappa^{\prime}+\kappa=\frac{1}{2}
$$

and assume that $s>1$ verifies, with $\rho=3 / 4, \delta=1 / 2$,

$$
(s-1) \kappa^{\prime},(s-1)(1-\rho+\kappa)<\rho-\delta-\kappa, \quad s \kappa^{\prime}<1-\delta .
$$

Let us set

$$
\tilde{\phi}\left(x, \xi^{\prime}\right)=-x_{0}\left\langle\mu \xi^{\prime}\right\rangle^{\kappa^{\prime}}+\phi\left(x, \xi^{\prime}\right) .
$$

We study in detail the operator $\operatorname{Op}^{0}\left(e^{\tilde{\phi}}\right) \mathrm{Op}^{0}(p) \mathrm{Op}^{1}\left(e^{-\tilde{\phi}}\right)$, where $\operatorname{Op}^{t}(p)$ is the $t$-quantization of $p$ (see Appendix B). In what follows it is assumed that $\left|x_{0}\right| \leq T$ with some $T>0$. Our goal in this section is Proposition 5.4.

In this section we apply the results in Appendices A and B with $a_{1}=1 / 2$, $a_{j}=1 / 4, j \geq 2, b_{j}=3 / 4, \delta=1 / 2, \rho=3 / 4$, and

$$
h=\left\langle\xi^{\prime}\right\rangle_{\mu}^{-1 / 4}, \quad k=\left\langle\mu \xi^{\prime}\right\rangle^{\kappa}\left\langle\xi^{\prime}\right\rangle_{\mu}^{\epsilon}
$$

where $0<\epsilon<1 / 4-\kappa$.

Recalling that $p$ is a polynomial in $\xi_{0}$,

$$
p(x, \xi)=-\xi_{0}^{2}+p_{1}\left(x, \xi^{\prime}\right) \xi_{0}+p_{2}\left(x, \xi^{\prime}\right),
$$

we apply Proposition B.1 $(\rho=3 / 4, \delta=1 / 2)$ to get

$$
\mathrm{Op}^{0}\left(e^{\tilde{\phi}}\right) \mathrm{Op}^{0}(p)=\mathrm{Op}^{0}\left(e^{\tilde{\phi}} q\right)+\mathrm{Op}^{0}\left(r_{0} \xi_{0}+r_{1}\right),
$$

where $r_{i}\left(x, \xi^{\prime}\right) \in S_{(s d)}\left(e^{-c\left\langle\mu \xi^{\prime}\right\rangle^{1 / 2 s}}, \bar{g}\right)$ with $d=5 / 2$ and

$$
q(x, \xi)=\sum_{|\beta|<5} \frac{1}{\beta !} \partial_{\eta^{\prime}}^{\beta} p_{(\beta)}\left(x_{0}, x^{\prime}-i \tilde{\Phi}\left(x, \xi^{\prime}, \eta^{\prime}\right), \xi\right)_{\eta^{\prime}=0}+R_{1}\left(x, \xi^{\prime}\right)+R_{0}\left(x, \xi^{\prime}\right) \xi_{0}
$$

with $R_{1}\left(x, \xi^{\prime}\right) \in \mu^{5 / 4} S_{(s)}\left(\left\langle\mu \xi^{\prime}\right\rangle, \bar{g}\right), R_{0}\left(x, \xi^{\prime}\right) \in \mu^{5 / 4} S_{(s)}(1, \bar{g})$, where

$$
\tilde{\Phi}\left(x, \xi^{\prime}, \eta^{\prime}\right)=\int_{0}^{1} \nabla_{\xi^{\prime}} \tilde{\phi}\left(x, \xi^{\prime}+\theta \eta^{\prime}\right) d \theta .
$$

We now conjugate $\mathrm{Op}^{1}\left(e^{-\tilde{\phi}}\right)$ on the right:

$$
\mathrm{Op}^{0}\left(e^{\tilde{\phi}} q\right) \mathrm{Op}^{1}\left(e^{-\tilde{\phi}}\right)+\mathrm{Op}^{0}\left(r_{0} \xi_{0}+r_{1}\right) \mathrm{Op}^{1}\left(e^{-\tilde{\phi}}\right) .
$$

If an operator $T$ is given by $T=\mathrm{Op}^{0}(p)$ with some $p \in S_{(s)}(m, g)$, then we abbreviate as $T=\mathrm{Op}^{0}\left(S_{(s)}(m, g)\right)$. Since $1 / 2 s>\kappa^{\prime}$, it follows from Proposition B.3 
that

$$
\mathrm{Op}^{0}\left(r_{0} \xi_{0}+r_{1}\right) \mathrm{Op}^{1}\left(e^{-\tilde{\phi}}\right)=\mu^{k} \mathrm{Op}^{0}\left(S_{\left(s d^{2}\right)}\left(\left\langle\mu \xi^{\prime}\right\rangle^{-k}, \bar{g}\right) \xi_{0}+S_{\left(s d^{2}\right)}\left(\left\langle\mu \xi^{\prime}\right\rangle^{-k}, \bar{g}\right)\right)
$$

for any $k \in \mathbb{N}$. Since $p_{i}\left(x, \xi^{\prime}\right) \in S_{(s)}\left(\left\langle\mu \xi^{\prime}\right\rangle^{i}, g_{0}\right)$, we see by Lemma A.2 that

$$
p_{i(\beta)}\left(x_{0}, x^{\prime}-i \tilde{\Phi}\left(x, \xi^{\prime}, \eta^{\prime}\right), \xi^{\prime}\right) \in S_{(s)}\left(\left\langle\mu \xi^{\prime}\right\rangle^{i}, g \mid E\right)
$$

for any $\beta$ because $\tilde{\Phi}\left(x, \xi^{\prime}, \eta^{\prime}\right) \in \mu^{3 / 4} S_{(s)}\left(\left\langle\mu \xi^{\prime}\right\rangle^{\kappa-3 / 4}, g \mid E\right)$, where $E=\left\{\left(x, \xi^{\prime}, \eta^{\prime}\right) \mid\right.$ $\left.\left|\eta^{\prime}\right|<\left|\xi^{\prime}\right| / 2\right\}$ (for the definition $S_{(s)}(m, g \mid E)$, see Appendix A). This proves that

$$
\partial_{\eta^{\prime}}^{\beta} p_{i(\beta)}\left(x_{0}, x^{\prime}-\tilde{\Phi}\left(x, \xi^{\prime}, \eta^{\prime}\right), \xi^{\prime}\right) \in \mu^{3(|\beta|+1) / 4} S_{(s)}\left(\left\langle\mu \xi^{\prime}\right\rangle^{i+\kappa-3 / 4}\left\langle\mu \xi^{\prime}\right\rangle^{-3|\beta| / 4}, g \mid E\right)
$$

for $|\beta| \geq 1$ by Lemma A.2 and hence

$$
q(x, \xi)=p\left(x_{0}, x^{\prime}-i \nabla_{\xi^{\prime}} \tilde{\phi}\left(x, \xi^{\prime}\right), \xi\right)+R_{0}\left(x, \xi^{\prime}\right) \xi_{0}+R_{1}\left(x, \xi^{\prime}\right)
$$

with $R_{0}\left(x, \xi^{\prime}\right) \in \mu^{5 / 4} S_{(s)}(1, \bar{g})$ and $R_{1}\left(x, \xi^{\prime}\right) \in \mu^{5 / 4} S_{(s)}\left(\left\langle\mu \xi^{\prime}\right\rangle, \bar{g}\right)$. From Proposition B.2 we see that

$$
\mathrm{Op}^{0}\left(e^{\tilde{\phi}}\left(R_{1}+R_{0} \xi_{0}\right)\right) \mathrm{Op}^{1}\left(e^{-\tilde{\phi}}\right)=\mu^{5 / 4} \mathrm{Op}^{0}\left(S_{\left(s d^{2}\right)}\left(\left\langle\mu \xi^{\prime}\right\rangle, \bar{g}\right)+S_{\left(s d^{2}\right)}(1, \bar{g}) \xi_{0}\right) .
$$

Thus we conclude that

$$
\begin{aligned}
\mathrm{Op}^{0}\left(e^{\tilde{\phi}}\right) \mathrm{Op}^{0}(p) \mathrm{Op}^{1}\left(e^{-\tilde{\phi}}\right)= & \mathrm{Op}^{0}\left(e^{\tilde{\phi}} p\left(x_{0}, x^{\prime}-i \nabla_{\xi^{\prime}} \tilde{\phi}\left(x, \xi^{\prime}\right), \xi\right)\right) \mathrm{Op}^{1}\left(e^{-\tilde{\phi}}\right) \\
& +\mu^{5 / 4} \mathrm{Op}^{0}\left(S\left(\left\langle\mu \xi^{\prime}\right\rangle, \bar{g}\right)+S(1, \bar{g}) \xi_{0}\right) .
\end{aligned}
$$

Let us set $q(x, \xi)=p\left(x_{0}, x^{\prime}-i \nabla_{\xi^{\prime}} \tilde{\phi}\left(x, \xi^{\prime}\right), \xi\right)$ and study $\mathrm{Op}^{0}\left(e^{\tilde{\phi}} q\right) \mathrm{Op}^{1}\left(e^{-\tilde{\phi}}\right)$. Since $q$ is a polynomial in $\xi_{0}$ of order 2 , we have $\operatorname{Op}^{0}\left(e^{\tilde{\phi}} q\right) \operatorname{Op}^{1}\left(e^{-\tilde{\phi}}\right)=\mathrm{Op}^{0}(b)+$ $\mu^{3 / 2} \operatorname{Op}^{0}\left(S_{\left(s d^{2}\right)}\left(\left\langle\mu \xi^{\prime}\right\rangle^{\kappa+1 / 2}, \bar{g}\right)\right)$, where

$$
\begin{aligned}
b(x, \xi)= & \lim _{\epsilon \rightarrow 0}(2 \pi)^{-n} \int e^{-i\left(x^{\prime}-y^{\prime}\right)\left(\xi^{\prime}-\eta^{\prime}\right)+\tilde{\phi}\left(x, \eta^{\prime}\right)-\tilde{\phi}\left(x_{0}, y^{\prime}, \eta^{\prime}\right)} \chi_{\epsilon}\left(y^{\prime}, \xi^{\prime}, \eta^{\prime}\right) \\
& \times q\left(x, \xi_{0}+i \partial_{x_{0}} \tilde{\phi}\left(x_{0}, y^{\prime}, \eta^{\prime}\right), \eta^{\prime}\right) d y^{\prime} d \eta^{\prime}
\end{aligned}
$$

because

$$
\mathrm{Op}^{0}\left(e^{\tilde{\phi}}\right) \operatorname{Op}^{1}\left(e^{-\tilde{\phi}} \partial_{x_{0}}^{2} \tilde{\phi}\right) \in \mu^{3 / 2} \operatorname{Op}^{0}\left(S_{\left(s d^{2}\right)}\left(\left\langle\mu \xi^{\prime}\right\rangle^{\kappa+1 / 2}, \bar{g}\right)\right)
$$

which follows from an assertion similar to Proposition B.2 because we have $\partial_{x_{0}}^{2} \tilde{\phi} \in$ $\mu^{3 / 2} S_{(s)}\left(\left\langle\mu \xi^{\prime}\right\rangle^{\kappa+1 / 2}, \bar{g}\right)$. Here we have set $\chi_{\epsilon}\left(y^{\prime}, \xi^{\prime}, \eta^{\prime}\right)=\chi\left(\epsilon y^{\prime}\right) \chi\left(\epsilon\left\langle\xi^{\prime}\right\rangle_{\mu}^{-1} \eta^{\prime}\right)$ with $\chi(t) \in \gamma_{0}^{(s)}\left(\mathbb{R}^{n}\right)$ such that $\chi(t)=1$ near $t=0$. Let $\Xi^{\prime}\left(x, y^{\prime}, \xi^{\prime}\right)=\xi^{\prime}+G^{\prime}\left(x, y^{\prime}, \xi^{\prime}\right)$ be the solution to

$$
\Xi^{\prime}-i \int_{0}^{1} \nabla_{x^{\prime}} \phi\left(x_{0}, x^{\prime}+\theta\left(y^{\prime}-x^{\prime}\right), \Xi^{\prime}\right) d \theta=\xi^{\prime}
$$

given by Proposition A.3 and

$$
J\left(x, y^{\prime}, \xi^{\prime}\right)=\operatorname{det}\left[\frac{\partial \Xi\left(x, y^{\prime}, \xi^{\prime}\right)}{\partial \xi^{\prime}}\right] .
$$


Applying Proposition B.2 we get

$$
\begin{aligned}
b(x, \xi)= & \sum_{|\alpha|<5} \frac{1}{\alpha !} \partial_{\xi^{\prime}}^{\alpha} D_{y^{\prime}}^{\alpha}\left[J\left(x, y^{\prime}, \xi^{\prime}\right)\right. \\
& \left.\times q\left(x, \xi_{0}+i\left(\partial_{x_{0}} \tilde{\phi}\right)\left(x_{0}, y^{\prime}, \Xi^{\prime}\left(x, y^{\prime}, \xi^{\prime}\right)\right), \Xi^{\prime}\left(x, y^{\prime}, \xi^{\prime}\right)\right)\right]_{y^{\prime}=x^{\prime}} \\
& +R_{1}\left(x, \xi^{\prime}\right)+R_{0}\left(x, \xi^{\prime}\right) \xi_{0}+R_{-1}\left(x, \xi^{\prime}\right) \xi_{0}^{2},
\end{aligned}
$$

where $R_{i}\left(x, \xi^{\prime}\right) \in \mu^{5 / 4} S\left(\left\langle\mu \xi^{\prime}\right\rangle^{i}, \bar{g}\right)$. We summarize by the following.

\section{PROPOSITION 5.1}

We have

$$
\begin{aligned}
& \mathrm{Op}^{0}\left(e^{\tilde{\phi}}\right) \mathrm{Op}^{0}(p) \mathrm{Op}^{1}\left(e^{-\tilde{\phi}}\right) \\
& \quad=\mathrm{Op}^{0}(b(x, \xi))+\mu^{5 / 4} \mathrm{Op}^{0}\left(S\left(\left\langle\mu \xi^{\prime}\right\rangle, \bar{g}\right)+S(1, \bar{g}) \xi_{0}+S\left(\left\langle\mu \xi^{\prime}\right\rangle^{-1}, \bar{g}\right) \xi_{0}^{2}\right)
\end{aligned}
$$

where

$$
\begin{aligned}
b(x, \xi)= & \sum_{|\alpha|<5} \frac{1}{\alpha !} \partial_{\xi^{\prime}}^{\alpha} D_{y^{\prime}}^{\alpha}\left[J ( x , y ^ { \prime } , \xi ^ { \prime } ) p \left(x_{0}, x^{\prime}-i\left(\nabla_{\xi^{\prime}} \tilde{\phi}\right)\left(x, \Xi^{\prime}\left(x, y^{\prime}, \xi^{\prime}\right)\right), \xi_{0}\right.\right. \\
& \left.\left.+i\left(\partial_{x_{0}} \tilde{\phi}\right)\left(x_{0}, y^{\prime}, \Xi^{\prime}\left(x, y^{\prime}, \xi^{\prime}\right)\right), \Xi^{\prime}\left(x, y^{\prime}, \xi^{\prime}\right)\right)\right]_{y^{\prime}=x^{\prime}}
\end{aligned}
$$

To simplify notation we denote by $S_{(s)}^{\#}(m, g)$ the set of $a\left(x^{\prime}, y^{\prime}, \xi^{\prime}\right)$ verifying

$$
\left[\partial_{x^{\prime}, y^{\prime}}^{\beta} \partial_{\xi^{\prime}}^{\alpha} a\left(x^{\prime}, y^{\prime}, \xi^{\prime}\right)\right]_{y^{\prime}=x^{\prime}} \in S_{(s)}\left(m\left(r^{-1}+\left\langle\xi^{\prime}\right\rangle_{\mu}^{1 / 4}\right)^{\beta_{1}}\left\langle\xi^{\prime}\right\rangle_{\mu}^{\left|\beta^{\prime}\right| / 4-3|\alpha| / 4}, g\right), \quad \forall \alpha, \beta .
$$

From Proposition A.3 with $\bar{k}\left(\xi^{\prime}\right)=\left\langle\mu \xi^{\prime}\right\rangle^{\kappa}, \Delta_{1}=r^{-2} w+r^{-1} w^{1 / 2}, \Delta_{j}=r^{-1} w^{1 / 2}$, $j \neq 1$, it follows that

$$
\begin{aligned}
& G_{j}\left(x, y^{\prime}, \xi^{\prime}\right) \in S_{(s)}\left(\left\langle\mu \xi^{\prime}\right\rangle^{\kappa}\left\langle\xi^{\prime}\right\rangle_{\mu}^{1 / 4}, \hat{g}\right), \quad j \neq 1, \\
& G_{1}\left(x, y^{\prime}, \xi^{\prime}\right) \in S_{(s)}\left(\left\langle\mu \xi^{\prime}\right\rangle^{\kappa}\left\langle\xi^{\prime}\right\rangle_{\mu}^{1 / 2}, \hat{g}\right), \\
& G_{j}\left(x, y^{\prime}, \xi^{\prime}\right) \in S_{(s)}^{\#}\left(\Delta_{j}\left\langle\mu \xi^{\prime}\right\rangle^{\kappa}, g\right),
\end{aligned}
$$

where $G^{\prime}\left(x, y^{\prime}, \xi^{\prime}\right)=\left(G_{1}, \ldots, G_{n}\right)$.

\section{LEMMA 5.1}

We have

$$
\begin{aligned}
w\left(x, \Xi^{\prime}\left(x, x^{\prime}, \xi^{\prime}\right)\right) & \in S_{(s)}\left(w\left(x, \xi^{\prime}\right), g\right), \quad r\left(x, \Xi^{\prime}\left(x, x^{\prime}, \xi^{\prime}\right)\right) \in S_{(s)}\left(r\left(x, \xi^{\prime}\right), g\right), \\
w\left(x, \Xi^{\prime}\left(x, x^{\prime}, \xi^{\prime}\right)\right) & =w\left(x, \xi^{\prime}\right)\left(1+O\left(\mu^{1 / 4}\right)\right), \\
\left(1+C \mu^{1 / 4}\right) r\left(x, \xi^{\prime}\right)^{2} & \geq\left|r\left(x, \Xi^{\prime}\left(x, x^{\prime}, \xi^{\prime}\right)\right)\right|^{2} \geq\left(1-C \mu^{1 / 4}\right) r\left(x, \xi^{\prime}\right)^{2} .
\end{aligned}
$$

Proof

Note that $w\left(x, \xi^{\prime}\right) \in S_{(s)}\left(w\left(x, \xi^{\prime}\right), g\right)$ and $r\left(x, \xi^{\prime}\right) \in S_{(s)}\left(r\left(x, \xi^{\prime}\right), g\right)$ by Lemma A.6. Since $\Xi^{\prime}\left(x, x^{\prime}, \xi^{\prime}\right)=\xi^{\prime}+G^{\prime}\left(x, x^{\prime}, \xi^{\prime}\right)$ taking into account (5.2), the first assertion 
follows from Lemma A.3. The second assertion follows from Corollary A.1. To check the third assertion it is enough to remark that

$$
\begin{aligned}
\left|r\left(x, \Xi^{\prime}\left(x, x^{\prime}, \xi^{\prime}\right)\right)\right|^{2} & =\left|\psi\left(x_{1}\right)^{2}+w\left(x, \Xi^{\prime}\left(x, x^{\prime}, \xi^{\prime}\right)\right)^{2}\right| \\
& =\left|\psi\left(x_{1}\right)^{2}+w\left(x, \xi^{\prime}\right)^{2}\left(1+O\left(\mu^{1 / 4}\right)\right)\right| .
\end{aligned}
$$

The next lemma is an immediate consequence of Corollary A.1, but we give a proof here.

\section{LEMMA 5.2}

Let $a\left(x^{\prime}, \xi^{\prime}\right) \in S_{(s)}\left(\left\langle\mu \xi^{\prime}\right\rangle^{m}, g\right)$ and $a\left(x^{\prime}+i y^{\prime}, \xi^{\prime}+i \eta^{\prime}\right)$ be the almost-analytic extension given by Proposition A.1 with $k\left(\xi^{\prime}\right)=\left\langle\mu \xi^{\prime}\right\rangle^{\kappa}\left\langle\xi^{\prime}\right\rangle_{\mu}^{\epsilon}, 0<\epsilon<1 / 4-\kappa$. Let $z\left(x^{\prime}, y^{\prime}, \xi^{\prime}, \eta^{\prime}\right) \in S_{(s)}\left(\left\langle\mu \xi^{\prime}\right\rangle^{\kappa}\left\langle\xi^{\prime}\right\rangle_{\mu}^{-3 / 4}, \hat{g} \mid E(k)\right), \quad \zeta\left(x^{\prime}, y^{\prime}, \xi^{\prime}, \eta^{\prime}\right) \in S_{(s)}\left(\left\langle\mu \xi^{\prime}\right\rangle^{\kappa}\left\langle\xi^{\prime}\right\rangle_{\mu}^{1 / 2}\right.$, $\hat{g} \mid E(k))$; then we have

$$
\begin{gathered}
a\left(x^{\prime}+z, \xi^{\prime}+\zeta\right)-\sum_{|\alpha+\beta|<\ell} \frac{1}{\alpha ! \beta !} \partial_{x^{\prime}}^{\beta} \partial_{\xi^{\prime}}^{\alpha} a\left(x^{\prime}, \xi^{\prime}\right) z^{\beta} \zeta^{\alpha} \\
\in \mu^{\ell / 4} S\left(\left\langle\mu \xi^{\prime}\right\rangle^{m-(1 / 4-\kappa-\epsilon) \ell}, \hat{g} \mid E(k)\right) .
\end{gathered}
$$

Proof

Let us denote $z=\hat{x}^{\prime}+i \hat{y}^{\prime}$ and $\zeta=\hat{\xi}^{\prime}+i \hat{\eta}^{\prime}$. With $\tilde{a}\left(x^{\prime}, y^{\prime}, \xi^{\prime}, \eta^{\prime}\right)=a\left(x^{\prime}+i y^{\prime}, \xi^{\prime}+\right.$ $\left.i \eta^{\prime}\right)$, we have from Taylor formula

$$
\begin{aligned}
& a\left(x^{\prime}+z, \xi^{\prime}+\zeta\right) \\
& =\sum_{|\alpha+\beta+\mu+\nu|<\ell} \frac{1}{\alpha ! \beta ! \mu ! \nu !} \partial_{x^{\prime}}^{\beta} \partial_{y^{\prime}}^{\nu} \partial_{\xi^{\prime}}^{\alpha} \partial_{\eta^{\prime}}^{\mu} \tilde{a}\left(x^{\prime}, 0, \xi^{\prime}, 0\right) \hat{x}^{\prime \beta} \hat{y}^{\prime \nu} \hat{\xi}^{\prime \alpha} \hat{\eta}^{\prime \mu} \\
& \quad+\sum_{|\alpha+\beta+\mu+\nu|=\ell} \frac{\ell}{\alpha ! \beta ! \mu ! \nu !} \int_{0}^{1}(1-\theta)^{\ell-1} \partial_{x^{\prime}}^{\beta} \partial_{y^{\prime}}^{\nu} \partial_{\xi^{\prime}}^{\alpha} \partial_{\eta^{\prime}}^{\mu} \\
& \quad \times \tilde{a}\left(x^{\prime}+\theta \hat{x}^{\prime}, \theta \hat{y}^{\prime}, \xi^{\prime}+\theta \hat{\xi}^{\prime}, \theta \hat{\eta}^{\prime}\right) d \theta \hat{x}^{\prime \beta} \hat{y}^{\prime \nu} \hat{\xi}^{\prime \alpha} \hat{\eta}^{\prime \mu} .
\end{aligned}
$$

Since $\left(\partial_{x_{j}}+i \partial_{y_{j}}\right) \tilde{a}\left(x^{\prime}, 0, \xi^{\prime}, 0\right)$ and $\left(\partial_{\xi_{j}}+i \partial_{\eta_{j}}\right) \tilde{a}\left(x^{\prime}, 0, \xi^{\prime}, 0\right)$ belong to the class $S_{(s)}\left(e^{-c\left\langle\mu \xi^{\prime}\right\rangle^{(1 / 4-\kappa-\epsilon) /(s-1)}}, g\right)$ by Proposition A.1, one can replace $\partial_{y_{j}}, \partial_{\eta_{j}}$ by $i \partial_{x_{j}}$, $i \partial_{\xi_{j}}$ with errors

$$
S_{(s)}\left(e^{-c\left\langle\mu \xi^{\prime}\right\rangle^{(1 / 4-\kappa-\epsilon) /(s-1)}}, g\right) .
$$

This shows that the first term in the right-hand side is

$$
\sum_{|\alpha+\beta|<\ell} \frac{1}{\alpha ! \beta !} \partial_{x^{\prime}}^{\beta} \partial_{\xi^{\prime}}^{\alpha} a\left(x^{\prime}, \xi^{\prime}\right) z^{\beta} \zeta^{\alpha}+S_{(s)}\left(e^{-c\left\langle\mu \xi^{\prime}\right\rangle^{(1 / 4-\kappa-\epsilon) /(s-1)}}, \hat{g} \mid E(k)\right)
$$

because $\partial_{x^{\prime}}^{\beta} \partial_{\xi^{\prime}}^{\alpha} \tilde{a}\left(x^{\prime}, 0, \xi^{\prime}, 0\right)=\partial_{x^{\prime}}^{\beta} \partial_{\xi^{\prime}}^{\alpha} a\left(x^{\prime}, \xi^{\prime}\right)$. From Lemma A.3 it follows that

$$
\int_{0}^{1}(\cdots) d \theta \in S_{(s)}\left(\left\langle\mu \xi^{\prime}\right\rangle^{m}\left\langle\xi^{\prime}\right\rangle_{\mu}^{|\beta+\nu| / 2-3|\alpha+\mu| / 4}, \hat{g} \mid E(k)\right) .
$$


On the other hand, since

$$
{\hat{x^{\prime}}}^{\beta}{\hat{y^{\prime}}}^{\nu} \hat{\xi}^{\prime}{\hat{\eta^{\prime}}}^{\mu} \in S_{(s)}\left(\left\langle\mu \xi^{\prime}\right\rangle^{\kappa \ell}\left\langle\xi^{\prime}\right\rangle_{\mu}^{-3|\beta+\nu| / 4+|\alpha+\mu| / 2}, \hat{g} \mid E(k)\right)
$$

for $|\alpha+\beta+\mu+\nu|=\ell$, we have

$$
\int_{0}^{1}(\cdots) d \theta{\hat{x^{\prime}}}^{\beta} \hat{y}^{\prime} \hat{\xi}^{\prime}{\hat{\eta^{\prime}}}^{\mu} \in \mu^{\ell / 4} S_{(s)}\left(\left\langle\mu \xi^{\prime}\right\rangle^{m-(1 / 4-\kappa-\epsilon) \ell}, \hat{g} \mid E(k)\right),
$$

which proves the desired assertion.

\section{LEMMA 5.3}

Let us denote $\Xi^{\prime}=\Xi^{\prime}\left(x, y^{\prime}, \xi^{\prime}\right)$. Then we have

$$
\begin{aligned}
& \partial_{\xi_{j}} \tilde{\phi}\left(x, \Xi^{\prime}\right)_{y^{\prime}=x^{\prime}}=\partial_{\xi_{j}} \tilde{\phi}\left(x, \xi^{\prime}\right)+\mu^{3 / 2} S\left(r^{-2} w\left\langle\mu \xi^{\prime}\right\rangle^{\kappa-5 / 4}, g\right)+\mu^{3 / 2} S\left(\left\langle\mu \xi^{\prime}\right\rangle^{-1}, g\right), \\
& \partial_{x_{j}} \phi\left(x, \Xi^{\prime}\right)_{y^{\prime}=x^{\prime}}=\partial_{x_{j}} \phi\left(x, \xi^{\prime}\right)+\mu^{1 / 2} S\left(r^{-2} w\left\langle\mu \xi^{\prime}\right\rangle^{\kappa-1 / 4}, g\right)+\mu S(1, g), \\
& \partial_{x_{1}} \phi\left(x, \Xi^{\prime}\right)_{y^{\prime}=x^{\prime}}=\partial_{x_{1}} \phi\left(x, \xi^{\prime}\right)+\mu^{3 / 4} S\left(r^{-3} w\left\langle\mu \xi^{\prime}\right\rangle^{\kappa-1 / 2}, g\right)+\mu S(1, g),
\end{aligned}
$$

and

$$
\partial_{x_{0}} \tilde{\phi}\left(x_{0}, y^{\prime}, \Xi^{\prime}\right)=\left\langle\mu \xi^{\prime}\right\rangle^{\kappa^{\prime}}+\mu S^{\#}\left(\left(r^{-1} w^{1 / 2}\left\langle\mu \xi^{\prime}\right\rangle^{\kappa}+r^{-1}\left\langle\mu \xi^{\prime}\right\rangle^{-1 / 2}\right), g\right) .
$$

Proof

Recall that $\partial_{\xi_{j}} \tilde{\phi}\left(x, \xi^{\prime}+i \eta^{\prime}\right)$ is the almost-analytic extension of $\partial_{\xi_{j}} \tilde{\phi}\left(x, \xi^{\prime}\right)$ with $k\left(\xi^{\prime}\right)=\left\langle\mu \xi^{\prime}\right\rangle^{\kappa}\left\langle\xi^{\prime}\right\rangle_{\mu}^{\epsilon}$. Since $\Xi^{\prime}\left(x, y^{\prime}, \xi^{\prime}\right)=\xi^{\prime}+G^{\prime}\left(x, y^{\prime}, \xi^{\prime}\right)$ it follows from Lemma 5.2 that

$$
\begin{aligned}
\partial_{\xi_{j}} \tilde{\phi}\left(x, \Xi^{\prime}\right)= & \partial_{\xi_{j}} \tilde{\phi}\left(x, \xi^{\prime}\right)+\sum_{1 \leq|\alpha|<\ell} \frac{1}{\alpha !} \partial_{\xi^{\prime}}^{\alpha} \partial_{\xi_{j}} \tilde{\phi}\left(x, \xi^{\prime}\right) G^{\prime}\left(x, y^{\prime}, \xi^{\prime}\right)^{\alpha} \\
& +\mu^{3 / 2} S\left(\left\langle\mu \xi^{\prime}\right\rangle^{-1}, \hat{g} \mid E^{0}(k) \times \mathbb{R}^{n}\right) .
\end{aligned}
$$

Since

$$
\partial_{\xi^{\prime}}^{\alpha} \partial_{\xi_{j}} \tilde{\phi}\left(x, \xi^{\prime}\right) \in S\left(\left\langle\mu \xi^{\prime}\right\rangle^{\kappa^{\prime}}\left\langle\xi^{\prime}\right\rangle_{\mu}^{-|\alpha|-1}, g\right)+S\left(r^{-1} w^{1 / 2}\left\langle\mu \xi^{\prime}\right\rangle^{\kappa}\left\langle\xi^{\prime}\right\rangle_{\mu}^{-1-3|\alpha| / 4}, g\right)
$$

and $G^{\prime}\left(x, x^{\prime}, \xi^{\prime}\right) \in S\left(\left(r^{-2} w+r^{-1} w^{1 / 2}\right)\left\langle\mu \xi^{\prime}\right\rangle^{\kappa}, g\right)$, we have the desired assertion. To prove the last two assertions of the first group it suffices to note that

$$
\partial_{\xi^{\prime}}^{\alpha} \partial_{x_{j}} \phi\left(x, \xi^{\prime}\right) \in S\left(\Delta_{j}\left\langle\mu \xi^{\prime}\right\rangle^{\kappa}\left\langle\xi^{\prime}\right\rangle_{\mu}^{-3|\alpha| / 4}, g\right)
$$

To prove the last assertion we note that

$$
\partial_{x_{0}} \phi\left(x_{0}, y^{\prime}, \xi^{\prime}\right) \in \mu S^{\#}\left(r^{-1} w^{1 / 2}\left\langle\mu \xi^{\prime}\right\rangle^{\kappa}, g\right)
$$

and $\left\langle\mu \Xi^{\prime}\right\rangle^{\kappa^{\prime}}=\left\langle\mu \xi^{\prime}\right\rangle^{\kappa^{\prime}}+\mu S^{\#}\left(r^{-1}\left\langle\mu \xi^{\prime}\right\rangle^{-1 / 2}, g\right)$, which follows from Lemma 5.2.

\section{LEMMA 5.4}

We have

$$
\begin{aligned}
\{\Lambda, \phi\}\left(x, \xi^{\prime}\right) & \in \mu S\left(\left\langle\mu \xi^{\prime}\right\rangle^{\kappa}, g\right), \\
\left\{\phi_{1}, \phi\right\}\left(x, \xi^{\prime}\right) & =2 r^{-2} w\left\langle\mu \xi^{\prime}\right\rangle^{\kappa}\left\{\phi_{1}, \psi\right\}+\mu S\left(\left\langle\mu \xi^{\prime}\right\rangle^{\kappa}, g\right),
\end{aligned}
$$




$$
\left\{\phi_{2}, \phi\right\}\left(x, \xi^{\prime}\right)=f\left(x, \xi^{\prime}\right)+\mu S\left(\left\langle\mu \xi^{\prime}\right\rangle^{\kappa}, g\right),
$$

where $f\left(x, \xi^{\prime}\right) \in \mu S\left(r^{-1} w^{1 / 2}\left\langle\mu \xi^{\prime}\right\rangle^{\kappa}, g\right)$.

\section{Proof}

We first note that

$$
\begin{aligned}
\{F, \phi\}= & \left\langle\mu \xi^{\prime}\right\rangle^{\kappa}\left[2 w \frac{\{F, \psi\}}{r^{2}}-2 \psi \frac{\{F, w\}}{r^{2}}\right]+\left\{F,\left\langle\mu \xi^{\prime}\right\rangle^{\kappa}\right\} S_{(s)}(1, g), \\
\{F, w\}= & 2 w^{-1} \phi_{1}^{3}\left\langle\mu \xi^{\prime}\right\rangle^{-4}\left\{F, \phi_{1}\right\} \\
& +2 w^{-1} \phi_{1}^{4}\left\langle\mu \xi^{\prime}\right\rangle^{-3}\left\{F,\left\langle\mu \xi^{\prime}\right\rangle^{-1}\right\}+2^{-1} w^{-1}\left\{F,\left\langle\xi^{\prime}\right\rangle_{\mu}^{-1}\right\} .
\end{aligned}
$$

The first assertion follows from (4.7) immediately. For the second assertion it suffices to note that $\left\{\phi_{1}, w\right\} \in \mu S\left(\left(w+w^{-1}\left\langle\xi^{\prime}\right\rangle_{\mu}^{-1}\right), g\right) \subset \mu S(w, g)$. To show the third assertion we note that $\left\{\phi_{2}, \psi\right\}=\{a, \psi\} \psi \in \mu S(r, g)$ and

$$
\left\{\phi_{2}, w\right\}=2 w^{-1} \phi_{1}^{3}\left\langle\mu \xi^{\prime}\right\rangle^{-4}\left\{\phi_{2}, \phi_{1}\right\}+\mu S(w, g) \in \mu S\left(w^{1 / 2}, g\right) .
$$

To simplify notation we set

$$
\begin{aligned}
\tilde{\Lambda}\left(x, y^{\prime}, \xi\right) & =\Lambda\left(x_{0}, x^{\prime}-i \nabla_{\xi^{\prime}} \tilde{\phi}\left(x, \Xi^{\prime}\right), \xi_{0}+i \partial_{x_{0}} \tilde{\phi}\left(x_{0}, y^{\prime}, \Xi^{\prime}\right), \Xi^{\prime}\right), \\
\tilde{\phi}_{1}\left(x, y^{\prime}, \xi^{\prime}\right) & =\phi_{1}\left(x_{0}, x^{\prime}-i \nabla_{\xi^{\prime}} \tilde{\phi}\left(x, \Xi^{\prime}\right), \Xi^{\prime}\right)
\end{aligned}
$$

with $\Xi^{\prime}=\Xi^{\prime}\left(x, y^{\prime}, \xi^{\prime}\right)$. We define $\tilde{M}\left(x, y^{\prime}, \xi\right), \tilde{\phi}_{2}\left(x, y^{\prime}, \xi^{\prime}\right), \tilde{\theta}\left(x, y^{\prime}, \xi^{\prime}\right)$ similarly.

PROPOSITION 5.2

Let $\tilde{\Lambda}\left(x, y^{\prime}, \xi\right), \tilde{\phi}_{1}\left(x, y^{\prime}, \xi^{\prime}\right), \tilde{\phi}_{2}\left(x, y^{\prime}, \xi^{\prime}\right)$, and $\tilde{\theta}\left(x, y^{\prime}, \xi^{\prime}\right)$ be as above. Then we have

$$
\begin{aligned}
\tilde{\Lambda}\left(x, y^{\prime}, \xi\right)= & \Lambda(x, \xi)-i\left\langle\mu \xi^{\prime}\right\rangle^{\kappa^{\prime}}+\mu S^{\#}\left(r^{-1} w^{1 / 2}\left\langle\mu \xi^{\prime}\right\rangle^{\kappa}, g\right) \\
& +\mu S^{\#}\left(\left(r^{-1}\left\langle\mu \xi^{\prime}\right\rangle^{-1 / 2}+\left\langle\mu \xi^{\prime}\right\rangle^{\kappa^{\prime}}\right), g\right), \\
\tilde{\Lambda}\left(x, x^{\prime}, \xi\right)= & \Lambda(x, \xi)-i\left\langle\mu \xi^{\prime}\right\rangle^{\kappa^{\prime}}+\mu S\left(\left\langle\mu \xi^{\prime}\right\rangle^{\kappa^{\prime}}, g\right)+\sqrt{\mu} S(1, g), \\
\tilde{\phi}_{1}\left(x, y^{\prime}, \xi^{\prime}\right)= & \phi_{1}\left(x, \xi^{\prime}\right)+\mu S^{\#}\left(r^{-1}\left\langle\mu \xi^{\prime}\right\rangle^{\kappa}, g\right)+\mu S^{\#}\left(\left\langle\mu \xi^{\prime}\right\rangle^{\kappa^{\prime}}, g\right), \\
\tilde{\phi}_{1}\left(x, x^{\prime}, \xi^{\prime}\right)= & \phi_{1}\left(x, \xi^{\prime}\right)+i\left\{\phi_{1}, \phi\right\}\left(x, \xi^{\prime}\right)+\mu^{5 / 4} S\left(r^{-2} w\left\langle\mu \xi^{\prime}\right\rangle^{\kappa}, g\right) \\
& +\mu S\left(\left\langle\mu \xi^{\prime}\right\rangle^{\kappa^{\prime}}, g\right), \\
\tilde{\phi}_{2}\left(x, y^{\prime}, \xi^{\prime}\right)= & \phi_{2}\left(x, \xi^{\prime}\right)+\mu S^{\#}\left(r^{-1} w^{1 / 2}\left\langle\mu \xi^{\prime}\right\rangle^{\kappa}, g\right)+\mu S^{\#}\left(\left\langle\mu \xi^{\prime}\right\rangle^{\kappa^{\prime}}, g\right), \\
\tilde{\phi}_{2}\left(x, x^{\prime}, \xi^{\prime}\right)= & \phi_{2}\left(x, \xi^{\prime}\right)+i\left\{\phi_{2}, \phi\right\}\left(x, \xi^{\prime}\right)+\mu^{5 / 4} S\left(r^{-2} w\left\langle\mu \xi^{\prime}\right\rangle^{\kappa-1 / 4}, g\right) \\
& +\mu S\left(\left\langle\mu \xi^{\prime}\right\rangle^{\kappa^{\prime}}, g\right), \\
\tilde{\theta}\left(x, y^{\prime}, \xi^{\prime}\right)= & \theta\left(x, \xi^{\prime}\right)+\mu S^{\#}\left(r^{-1} w^{1 / 2}\left\langle\mu \xi^{\prime}\right\rangle^{\kappa}, g\right)+\mu S^{\#}\left(\left\langle\mu \xi^{\prime}\right\rangle^{\kappa^{\prime}}, g\right), \\
\tilde{\theta}\left(x, x^{\prime}, \xi^{\prime}\right)= & \theta\left(x, \xi^{\prime}\right)+i f\left(x, \xi^{\prime}\right)+\mu^{5 / 4} S\left(r^{-1} w^{1 / 2}\left\langle\mu \xi^{\prime}\right\rangle^{2 \kappa-1 / 4}, g\right) \\
& +\mu S\left(\left\langle\mu \xi^{\prime}\right\rangle^{\kappa^{\prime}}, g\right),
\end{aligned}
$$

where $f \in \mu S\left(r^{-1} w^{1 / 2}\left\langle\mu \xi^{\prime}\right\rangle^{\kappa}, g\right)$ is real. 
Proof

Recall that $\Xi^{\prime}=\xi^{\prime}+G\left(x, y^{\prime}, \xi^{\prime}\right)$. From Lemma 5.2 (or rather from its proof) and (5.2), we have

$$
\begin{aligned}
\tilde{\Lambda}\left(x, y^{\prime}, \xi\right)= & \Lambda\left(x_{0}, x^{\prime}-i \nabla_{\xi^{\prime}} \tilde{\phi}\left(x, \Xi^{\prime}\right), \xi_{0}+i \partial_{x_{0}} \tilde{\phi}\left(x_{0}, y^{\prime}, \Xi^{\prime}\right), \Xi^{\prime}\right) \\
= & \Lambda(x, \xi)+i \partial_{x_{0}} \tilde{\phi}\left(x_{0}, y^{\prime}, \Xi^{\prime}\right) \\
& +\sum_{|\alpha+\beta|=1} \partial_{x^{\prime}}^{\beta} \partial_{\xi^{\prime}}^{\alpha} \Lambda(x, \xi)\left(-i \nabla_{\xi^{\prime}} \tilde{\phi}\left(x, \Xi^{\prime}\right)\right)^{\beta} G^{\prime}\left(x, y^{\prime}, \xi^{\prime}\right)^{\alpha} \\
& +\sum_{2 \leq|\alpha+\beta|<\ell} \frac{1}{\alpha ! \beta !} \partial_{x^{\prime}}^{\beta} \partial_{\xi^{\prime}}^{\alpha} \Lambda(x, \xi)\left(-i \nabla_{\xi^{\prime}} \tilde{\phi}\left(x, \Xi^{\prime}\right)\right)^{\beta} G^{\prime}\left(x, y^{\prime}, \xi^{\prime}\right)^{\alpha} \\
& +\mu^{\ell / 4} S^{\#}\left(\left\langle\mu \xi^{\prime}\right\rangle^{\kappa}, g\right)
\end{aligned}
$$

taking $\ell$ large if necessary. Noting (4.7), $\partial_{x^{\prime}}^{\beta} \partial_{\xi^{\prime}}^{\alpha} \Lambda(x, \xi) \in S\left(\left\langle\mu \xi^{\prime}\right\rangle\left\langle\xi^{\prime}\right\rangle_{\mu}^{-|\alpha|}, g\right)$, and $\nabla_{\xi^{\prime}} \phi\left(x, \Xi^{\prime}\right) \in S^{\#}\left(\left(1+r^{-1} w^{1 / 2}\right)\left\langle\mu \xi^{\prime}\right\rangle^{\kappa}\left\langle\xi^{\prime}\right\rangle_{\mu}^{-1}, g\right)$, it follows from (5.2) that

$$
\begin{aligned}
& i \partial_{x_{0}} \tilde{\phi}\left(x_{0}, y^{\prime}, \Xi^{\prime}\right)+\sum_{|\alpha+\beta|=1} \partial_{x^{\prime}}^{\beta} \partial_{\xi^{\prime}}^{\alpha} \Lambda(x, \xi)\left(-i \nabla_{\xi^{\prime}} \tilde{\phi}\left(x, \Xi^{\prime}\right)\right)^{\beta} G^{\prime}\left(x, y^{\prime}, \xi^{\prime}\right)^{\alpha} \\
& \in \mu S^{\#}\left(\left(r^{-1} w^{1 / 2}\left\langle\mu \xi^{\prime}\right\rangle^{\kappa}+\left\langle\mu \xi^{\prime}\right\rangle^{\kappa^{\prime}}\right), g\right) .
\end{aligned}
$$

Thanks to (4.7) similar arguments show

$$
\sum_{2 \leq|\alpha+\beta|<\ell} \cdots \in \mu^{5 / 4} S^{\#}\left(\left\langle\mu \xi^{\prime}\right\rangle^{\kappa}, g\right) .
$$

Thus we have the assertion about $\tilde{\Lambda}\left(x, y^{\prime}, \xi\right)$. We turn to the assertion for $\tilde{\phi}_{1}(x$, $\left.y^{\prime}, \xi^{\prime}\right)$. The same arguments as above show that

$$
\begin{aligned}
& \sum_{|\alpha+\beta|=1} \phi_{1(\beta)}^{(\alpha)}\left(x, \xi^{\prime}\right)\left(-i \nabla_{\xi^{\prime}} \tilde{\phi}\left(x, \Xi^{\prime}\right)\right)^{\beta} G^{\prime}\left(x, y^{\prime}, \xi^{\prime}\right)^{\alpha} \\
& \quad \in \mu S^{\#}\left(\left(r^{-1}\left\langle\mu \xi^{\prime}\right\rangle^{\kappa}+\left\langle\mu \xi^{\prime}\right\rangle^{\kappa^{\prime}}\right), g\right) .
\end{aligned}
$$

We turn to the term $|\alpha+\beta| \geq 2$. It is easy to see that

$$
\begin{aligned}
& \sum_{2 \leq|\alpha+\beta|<\ell} \frac{1}{\alpha ! \beta !} \phi_{1(\beta)}^{(\alpha)}\left(x, \xi^{\prime}\right)\left(-i \nabla_{\xi^{\prime}} \tilde{\phi}\left(x, \Xi^{\prime}\right)\right)^{\beta} G^{\prime}\left(x, y^{\prime}, \xi^{\prime}\right)^{\alpha} \\
& \quad \in \mu S^{\#}\left(\left(\left\langle\mu \xi^{\prime}\right\rangle^{\kappa}+r^{-1}\left\langle\mu \xi^{\prime}\right\rangle^{\kappa-1 / 4}\right), g\right)
\end{aligned}
$$

and hence the result. We show the assertion for $\tilde{\phi}_{2}\left(x, y^{\prime}, \xi^{\prime}\right)$. Let $|\alpha+\beta|=1$. Noting that $\phi_{2}^{(\alpha)}\left(x, \xi^{\prime}\right) \in S\left(r\left\langle\mu \xi^{\prime}\right\rangle\left\langle\xi^{\prime}\right\rangle_{\mu}^{-|\alpha|}, g\right)$, the same arguments as above show that

$$
\begin{aligned}
& \sum_{|\alpha+\beta|=1} \phi_{2(\beta)}^{(\alpha)}\left(x, \xi^{\prime}\right)\left(-i \nabla_{\xi^{\prime}} \tilde{\phi}\left(x, \Xi^{\prime}\right)\right)^{\beta} G^{\prime}\left(x, y^{\prime}, \xi^{\prime}\right)^{\alpha} \\
& \quad \in \mu S^{\#}\left(\left(r^{-1} w^{1 / 2}\left\langle\mu \xi^{\prime}\right\rangle^{\kappa}+\left\langle\mu \xi^{\prime}\right\rangle^{\kappa^{\prime}}\right), g\right) .
\end{aligned}
$$


We check the term $\sum_{|\alpha+\beta| \geq 2} \cdots$. It is easy to see that

$$
\sum_{2 \leq|\alpha+\beta|<\ell} \frac{1}{\alpha ! \beta !} \phi_{2(\beta)}^{(\alpha)}\left(x, \xi^{\prime}\right)\left(-i \nabla_{\xi^{\prime}} \tilde{\phi}\left(x, \Xi^{\prime}\right)\right)^{\beta} G^{\prime}\left(x, y^{\prime}, \xi^{\prime}\right)^{\alpha} \in \mu S^{\#}\left(\left\langle\mu \xi^{\prime}\right\rangle^{\kappa}, g\right)
$$

and hence the result. To check the assertion about $\tilde{\theta}\left(x, y^{\prime}, \xi^{\prime}\right)$ it suffices to note $\theta\left(x, \xi^{\prime}\right) \in S\left(\left\langle\mu \xi^{\prime}\right\rangle, g_{0}\right) \cap S\left(w\left\langle\mu \xi^{\prime}\right\rangle, g\right)$ and Lemma 5.2.

We prove the assertion for $\tilde{\Lambda}\left(x, x^{\prime}, \xi\right)$. Since $\Xi^{\prime}\left(x, x^{\prime}, \xi^{\prime}\right)=\xi^{\prime}+i \nabla_{x^{\prime}} \phi\left(x, \Xi^{\prime}\right)$, we see with $\Xi^{\prime}=\Xi^{\prime}\left(x, x^{\prime}, \xi^{\prime}\right)$,

$$
\begin{aligned}
\tilde{\Lambda} & \left(x, x^{\prime}, \xi\right) \\
= & \Lambda\left(x_{0}, x^{\prime}-i \nabla_{\xi^{\prime}} \tilde{\phi}\left(x, \Xi^{\prime}\right), \xi_{0}+i \partial_{x_{0}} \tilde{\phi}\left(x, \Xi^{\prime}\right), \xi^{\prime}+i \nabla_{x^{\prime}} \phi\left(x, \Xi^{\prime}\right)\right) \\
= & \Lambda(x, \xi)+i \partial_{x_{0}} \tilde{\phi}\left(x, \Xi^{\prime}\right)+\sum_{1 \leq|\alpha+\beta|<\ell} \frac{1}{\alpha ! \beta !} \partial_{x^{\prime}}^{\beta} \partial_{\xi^{\prime}}^{\alpha} \Lambda(x, \xi)\left(-i \nabla_{\xi^{\prime}} \tilde{\phi}\right)^{\beta}\left(i \nabla_{x^{\prime}} \phi\right)^{\alpha} \\
& \quad+\mu^{5 / 4} S\left(\left\langle\mu \xi^{\prime}\right\rangle^{\kappa}, g\right)
\end{aligned}
$$

by Lemma 5.2 taking $\ell$ large. From Lemma 5.3 and (4.7) it follows that

$$
\sum_{2 \leq|\alpha+\beta|<\ell} \frac{1}{\alpha ! \beta !} \partial_{x^{\prime}}^{\beta} \partial_{\xi^{\prime}}^{\alpha} \Lambda(x, \xi)\left(-i \nabla_{\xi^{\prime}} \tilde{\phi}\right)^{\beta}\left(i \nabla_{x^{\prime}} \phi\right)^{\alpha} \in \mu^{5 / 4} S\left(\left\langle\mu \xi^{\prime}\right\rangle^{\kappa^{\prime}}, g\right) \text {. }
$$

Since $\xi^{\prime}=\Xi^{\prime}-i \nabla_{x^{\prime}} \phi\left(x, \Xi^{\prime}\right)$, we see that

$$
\begin{aligned}
i \partial_{x_{0}} \tilde{\phi} & +\sum_{|\alpha+\beta|=1} \partial_{x^{\prime}}^{\beta} \partial_{\xi^{\prime}}^{\alpha} \Lambda(x, \xi)\left(-i \nabla_{\xi^{\prime}} \tilde{\phi}\right)^{\beta}\left(i \nabla_{x^{\prime}} \phi\right)^{\alpha} \\
= & -i\left\langle\mu \Xi^{\prime}\right\rangle^{\kappa^{\prime}}+\sum_{|\alpha+\beta|=1} \partial_{x^{\prime}}^{\beta} \partial_{\xi}^{\alpha} \Lambda(x, \xi)\left(-i \nabla_{\xi^{\prime}} \phi\right)^{\beta}\left(i \nabla_{x} \phi\right)^{\alpha}+\mu S\left(\left\langle\mu \xi^{\prime}\right\rangle^{\kappa^{\prime}}, g\right) \\
= & -i\left\langle\mu \Xi^{\prime}\right\rangle^{\kappa^{\prime}}+i\{\Lambda, \phi\}\left(x, \Xi^{\prime}\right) \\
& +\sum_{1 \leq|\gamma|<\ell,|\alpha+\beta|=1} \frac{1}{\gamma !} \partial_{\xi^{\prime}}^{\gamma} \partial_{x^{\prime}}^{\beta} \partial_{\xi}^{\alpha} \Lambda\left(x, \xi_{0}, \Xi^{\prime}\right)\left(-i \nabla_{x^{\prime}} \phi\right)^{\gamma}\left(-i \nabla_{\xi^{\prime}} \phi\right)^{\beta}\left(i \nabla_{x} \phi\right)^{\alpha} \\
& +\mu S\left(\left\langle\mu \xi^{\prime}\right\rangle^{\kappa^{\prime}}, g\right)
\end{aligned}
$$

by Lemma 5.2 again. From Lemma 5.3 and (4.7) it is easy to check that the third term in the right-hand side is in $\mu^{5 / 4} S\left(\left\langle\mu \xi^{\prime}\right\rangle^{\kappa}, g\right)$. We now consider $\{\Lambda, \phi\}\left(x, \Xi^{\prime}\right)$. Note that

$$
\begin{aligned}
\{\Lambda, \phi\}\left(x, \Xi^{\prime}\right)= & \{\Lambda, \phi\}\left(x, \xi^{\prime}\right)+\sum_{1 \leq|\gamma|<\ell} \frac{1}{\gamma !}\left(\partial_{\xi^{\prime}}^{\gamma}\{\Lambda, \phi\}\right)\left(x, \xi^{\prime}\right)\left(i \nabla_{x^{\prime}} \phi\right)^{\gamma} \\
& +\mu^{5 / 4} S\left(\left\langle\mu \xi^{\prime}\right\rangle^{\kappa}, g\right) .
\end{aligned}
$$

Thanks to $\{\Lambda, \phi\}\left(x, \xi^{\prime}\right) \in \mu S\left(\left\langle\mu \xi^{\prime}\right\rangle^{\kappa}, g\right)$ by Lemma 5.4 , one sees easily that the second term in the right-hand side is in $\mu^{5 / 4} S\left(\left\langle\mu \xi^{\prime}\right\rangle^{\kappa}, g\right)$, and hence we have $\{\Lambda, \phi\}\left(x, \Xi^{\prime}\right) \in \mu S\left(\left\langle\mu \xi^{\prime}\right\rangle^{\kappa}, g\right)$. These prove the assertion. 
Noting the fact

$$
\left\{\begin{array}{l}
\nabla_{\xi^{\prime}} \phi\left(x, \Xi^{\prime}\left(x, x^{\prime}, \xi^{\prime}\right)\right)=\nabla_{\xi^{\prime}} \phi\left(x, \xi^{\prime}\right)+\mu^{3 / 2} S\left(r^{-2} w\left\langle\mu \xi^{\prime}\right\rangle^{2 \kappa-3 / 2}, g\right), \\
G_{1}\left(x, x^{\prime}, \xi^{\prime}\right)=i \partial_{x_{1}} \phi\left(x, \xi^{\prime}\right)+\mu^{3 / 4} S\left(r^{-3} w\left\langle\mu \xi^{\prime}\right\rangle^{2 \kappa-3 / 4}, g\right), \\
G_{j}\left(x, x^{\prime}, \xi^{\prime}\right)=i \partial_{x_{j}} \phi\left(x, \xi^{\prime}\right)+\mu^{1 / 2} S\left(r^{-2} w\left\langle\mu \xi^{\prime}\right\rangle^{2 \kappa-1 / 2}, g\right),
\end{array}\right.
$$

which follows from Lemma 5.2 or rather its proof because we have $G^{\prime}\left(x, x^{\prime}, \xi^{\prime}\right)=$ $i \nabla_{x^{\prime}} \phi\left(x_{0}, x^{\prime}, \xi^{\prime}+G^{\prime}\left(x, x^{\prime}, \xi^{\prime}\right)\right)$, the assertions on $\tilde{\phi}_{1}\left(x, x^{\prime}, \xi^{\prime}\right), \tilde{\phi}_{2}\left(x, x^{\prime}, \xi^{\prime}\right)$ and $\tilde{\theta}_{1}\left(x, x^{\prime}, \xi^{\prime}\right)$ are checked by similar easier arguments.

Let us denote

$$
j\left(x, \xi^{\prime}\right)=\sum_{|\alpha|<5} \frac{1}{\alpha !} D_{y^{\prime}}^{\alpha} \partial_{\xi^{\prime}}^{\alpha} J\left(x, y^{\prime}, \xi^{\prime}\right)_{y^{\prime}=x^{\prime}} .
$$

Let $a_{i}\left(x^{\prime}, \xi^{\prime}\right) \in S\left(\left\langle\mu \xi^{\prime}\right\rangle^{m_{i}}, g\right)$; then there exists $b \in S\left(\left\langle\mu \xi^{\prime}\right\rangle^{m_{1}+m_{2}}, g\right)$ such that $\mathrm{Op}^{t}\left(a_{1}\right) \mathrm{Op}^{t}\left(a_{2}\right)=\mathrm{Op}^{t}(b)$. We denote $b$ as

$$
b=a_{1} \# a_{2} .
$$

\section{PROPOSITION 5.3}

We have

$$
\begin{aligned}
& \sum_{|\alpha|<5} \frac{1}{\alpha !} D_{y^{\prime}}^{\alpha} \partial_{\xi^{\prime}}^{\alpha}\left[\tilde{M}\left(x, y^{\prime}, \xi\right) \tilde{\Lambda}\left(x, y^{\prime}, \xi\right) J\left(x, y^{\prime}, \xi^{\prime}\right)\right]_{y^{\prime}=x^{\prime}} \\
& \quad=\left[\tilde{M}\left(x, x^{\prime}, \xi\right) \tilde{\Lambda}\left(x, x^{\prime}, \xi\right)\right] \# j+C_{1}\left(x, \xi^{\prime}\right) \tilde{\Lambda}\left(x, x^{\prime}, \xi\right)+\mu S\left(\left\langle\mu \xi^{\prime}\right\rangle, g\right)
\end{aligned}
$$

with $C_{1}\left(x, \xi^{\prime}\right) \in \mu S\left(\left\langle\mu \xi^{\prime}\right\rangle^{\kappa}, g\right)$,

$$
\begin{aligned}
& \sum_{|\alpha|<5} \frac{1}{\alpha !} D_{y^{\prime}}^{\alpha} \partial_{\xi^{\prime}}^{\alpha}\left[\tilde{\phi}_{1}\left(x, y^{\prime}, \xi^{\prime}\right) \tilde{\Lambda}\left(x, y^{\prime}, \xi\right) J\left(x, y^{\prime}, \xi^{\prime}\right)\right]_{y^{\prime}=x^{\prime}} \\
& \quad=\left[\tilde{\phi}_{1}\left(x, x^{\prime}, \xi^{\prime}\right) \tilde{\Lambda}\left(x, x^{\prime}, \xi\right)\right] \# j+C_{2}\left(x, \xi^{\prime}\right) \tilde{\Lambda}\left(x, x^{\prime}, \xi\right)+\mu S\left(\left\langle\mu \xi^{\prime}\right\rangle, g\right)
\end{aligned}
$$

with $C_{2} \in \mu^{5 / 4} S\left(r^{-2} w\left\langle\mu \xi^{\prime}\right\rangle^{\kappa-1 / 4}, g\right)+\mu S\left(\left\langle\mu \xi^{\prime}\right\rangle^{\kappa^{\prime}}, g\right)$, and

$$
\begin{aligned}
\sum_{|\alpha|<5} \frac{1}{\alpha !} D_{y^{\prime}}^{\alpha} \partial_{\xi^{\prime}}^{\alpha}\left[\tilde{\phi}_{2}\left(x, y^{\prime}, \xi^{\prime}\right)^{2} J\left(x, y^{\prime}, \xi^{\prime}\right)\right]_{y^{\prime}=x^{\prime}}= & \tilde{\phi}_{2}\left(x, x^{\prime}, \xi^{\prime}\right)^{2} \# j\left(x, \xi^{\prime}\right) \\
& +\mu S\left(\left\langle\mu \xi^{\prime}\right\rangle, g\right), \\
\sum_{|\alpha|<5} \frac{1}{\alpha !} D_{y^{\prime}}^{\alpha} \partial_{\xi^{\prime}}^{\alpha}\left[\tilde{\theta}\left(x, y^{\prime}, \xi^{\prime}\right)^{2} J\left(x, y^{\prime}, \xi^{\prime}\right)\right]_{y^{\prime}=x^{\prime}}= & \tilde{\theta}\left(x, x^{\prime}, \xi^{\prime}\right)^{2} \# j\left(x, \xi^{\prime}\right) \\
& +\mu S\left(\left\langle\mu \xi^{\prime}\right\rangle, g\right) .
\end{aligned}
$$

In what follows, in this section, to simplify notation we denote by $C_{1}, C_{2}, R$ symbols belonging to (or the class itself)

$$
\mu^{3 / 4} S\left(\left\langle\mu \xi^{\prime}\right\rangle^{\kappa^{\prime}}, g\right), \quad \mu^{5 / 4} S\left(r^{-2} w\left\langle\mu \xi^{\prime}\right\rangle^{\kappa-1 / 4}, g\right), \quad \mu S\left(\left\langle\mu \xi^{\prime}\right\rangle, g\right),
$$

respectively. To show the proposition we first prove the following. 


\section{LEMMA 5.5}

We have

$$
\begin{aligned}
& \partial_{y^{\prime}}^{\alpha} \partial_{\xi^{\prime}}^{\alpha}\left(\tilde{M}\left(x, y^{\prime}, \xi\right) \tilde{\Lambda}\left(x, y^{\prime}, \xi\right) J\left(x, y^{\prime}, \xi^{\prime}\right)\right)_{y^{\prime}=x^{\prime}} \\
& =\tilde{M}\left(x, x^{\prime}, \xi\right) \tilde{\Lambda}\left(x, x^{\prime}, \xi\right) \partial_{y^{\prime}}^{\alpha} \partial_{\xi^{\prime}}^{\alpha} J\left(x, y^{\prime}, \xi^{\prime}\right)_{y^{\prime}=x^{\prime}}+C_{1}\left(x, \xi^{\prime}\right) \tilde{\Lambda}\left(x, x^{\prime}, \xi\right)+R \\
& \partial_{y^{\prime}}^{\alpha} \partial_{\xi^{\prime}}^{\alpha}\left(\tilde{\phi}_{1}\left(x, y^{\prime}, \xi^{\prime}\right) \tilde{\Lambda}\left(x, y^{\prime}, \xi\right) J\left(x, y^{\prime}, \xi^{\prime}\right)\right)_{y^{\prime}=x^{\prime}} \\
& =\tilde{\phi}_{1}\left(x, x^{\prime}, \xi^{\prime}\right) \tilde{\Lambda}\left(x, x^{\prime}, \xi\right) \partial_{y^{\prime}}^{\alpha} \partial_{\xi^{\prime}}^{\alpha} J\left(x, y^{\prime}, \xi^{\prime}\right)_{y^{\prime}=x^{\prime}} \\
& \quad+C_{1}\left(x, \xi^{\prime}\right) \tilde{\Lambda}\left(x, x^{\prime}, \xi^{\prime}\right)+C_{2}\left(x, \xi^{\prime}\right) \tilde{\Lambda}\left(x, x^{\prime}, \xi\right)+R \\
& \partial_{y^{\prime}}^{\alpha} \partial_{\xi^{\prime}}^{\alpha}\left(\tilde{\phi}_{2}\left(x, y^{\prime}, \xi^{\prime}\right)^{2} J\left(x, y^{\prime}, \xi^{\prime}\right)\right)_{y^{\prime}=x^{\prime}} \\
& =\tilde{\phi}_{2}\left(x, x^{\prime}, \xi^{\prime}\right)^{2} \partial_{y^{\prime}}^{\alpha} \partial_{\xi^{\prime}}^{\alpha} J\left(x, y^{\prime}, \xi^{\prime}\right)_{y^{\prime}=x^{\prime}}+R \\
& \partial_{y^{\prime}}^{\alpha} \partial_{\xi^{\prime}}^{\alpha}\left(\tilde{\theta}\left(x, y^{\prime}, \xi^{\prime}\right)^{2} J\left(x, y^{\prime}, \xi^{\prime}\right)\right)_{y^{\prime}=x^{\prime}} \\
& =\tilde{\theta}\left(x, x^{\prime}, \xi^{\prime}\right)^{2} \partial_{y^{\prime}}^{\alpha} \partial_{\xi^{\prime}}^{\alpha} J\left(x, y^{\prime}, \xi^{\prime}\right)_{y^{\prime}=x^{\prime}}+R .
\end{aligned}
$$

Proof

From Proposition 5.2 and Lemma 5.4 one can write

$$
\begin{gathered}
\tilde{\Lambda}\left(x, y^{\prime}, \xi\right)=\Lambda(x, \xi)-i\left\langle\mu \xi^{\prime}\right\rangle^{\kappa^{\prime}}+\lambda\left(x, y^{\prime}, \xi^{\prime}\right), \\
\tilde{M}\left(x, y^{\prime}, \xi\right)=M(x, \xi)-i\left\langle\mu \xi^{\prime}\right\rangle^{\kappa^{\prime}}+m\left(x, y^{\prime}, \xi^{\prime}\right)
\end{gathered}
$$

with

$$
\lambda\left(x, y^{\prime}, \xi^{\prime}\right), m\left(x, y^{\prime}, \xi^{\prime}\right) \in \mu S^{\#}\left(\left(r^{-1} w^{1 / 2}\left\langle\mu \xi^{\prime}\right\rangle^{\kappa}+r^{-1}\left\langle\mu \xi^{\prime}\right\rangle^{-1 / 2}+\left\langle\mu \xi^{\prime}\right\rangle^{\kappa^{\prime}}\right), g\right) .
$$

Recall that $J\left(x, y^{\prime}, \xi^{\prime}\right) \in S^{\#}(1, g)$ and, moreover,

$$
\begin{aligned}
\partial_{\xi^{\prime}}^{\beta} \partial_{y^{\prime}}^{\alpha} & J\left(x, y^{\prime}, \xi^{\prime}\right)_{y^{\prime}=x^{\prime}} \in S\left(\left(r^{-2} w+r^{-1} w^{1 / 2}\right)\left\langle\mu \xi^{\prime}\right\rangle^{\kappa}\left\langle\xi^{\prime}\right\rangle_{\mu}^{-3 / 4}\right. \\
& \left.\times\left(r^{-1}+\left\langle\xi^{\prime}\right\rangle_{\mu}^{1 / 4}\right)^{\alpha_{1}}\left\langle\xi^{\prime}\right\rangle_{\mu}^{-3|\beta| / 4+\left(|\alpha|-\alpha_{1}\right) / 4}, g\right) \\
\subset & S\left(\left(r^{-2} w+r^{-1} w^{1 / 2}\right)\left\langle\mu \xi^{\prime}\right\rangle^{\kappa}\left\langle\xi^{\prime}\right\rangle_{\mu}^{-3(1+|\beta|) / 4+\left(|\alpha|+\alpha_{1}\right) / 4}, g\right) \\
\subset & S\left(\left(r^{-1}+\left\langle\xi^{\prime}\right\rangle_{\mu}^{1 / 4}\right)\left\langle\mu \xi^{\prime}\right\rangle^{\kappa}\left\langle\xi^{\prime}\right\rangle_{\mu}^{|\alpha| / 2-3(1+|\beta|) / 4}, g\right) \\
\subset & \mu^{1 / 4} S\left(\left\langle\mu \xi^{\prime}\right\rangle^{\kappa-1 / 4}\left\langle\xi^{\prime}\right\rangle_{\mu}^{|\alpha| / 2-3|\beta| / 4}, g\right)
\end{aligned}
$$

with $\alpha=\left(\alpha_{1}, \alpha^{\prime}\right)$ for $|\beta+\alpha| \geq 1$. Let us set $\bar{\Lambda}=\Lambda(x, \xi)-i\left\langle\mu \xi^{\prime}\right\rangle^{\kappa^{\prime}}$ and $\bar{M}=$ $M(x, \xi)-i\left\langle\mu \xi^{\prime}\right\rangle^{\kappa^{\prime}}$ and consider

$$
\tilde{M} \tilde{\Lambda} J=\bar{M} \bar{\Lambda} J+m \bar{\Lambda} J+\lambda \bar{M} J+m \lambda J .
$$

It is clear that $\partial_{\xi^{\prime}}^{\alpha} \partial_{y^{\prime}}^{\alpha}(m \lambda J)_{y^{\prime}=x^{\prime}} \in R$. Here we note that $\bar{M}(x, \xi)=\bar{\Lambda}(x, \xi)+$ $2 k \phi_{1}\left(x, \xi^{\prime}\right)^{3}\left\langle\mu \xi^{\prime}\right\rangle^{-2}$ and hence

$$
\lambda \bar{M} J=\lambda \bar{\Lambda} J+\phi_{1}^{3}\left\langle\mu \xi^{\prime}\right\rangle^{-2} \lambda J .
$$

Noting that $\phi_{1}^{3}\left\langle\mu \xi^{\prime}\right\rangle^{-2} \in S\left(w^{3 / 2}\left\langle\mu \xi^{\prime}\right\rangle, g\right)$, we see easily that

$$
\partial_{\xi^{\prime}}^{\alpha} \partial_{y^{\prime}}^{\alpha}\left(\phi_{1}^{3}\left\langle\mu \xi^{\prime}\right\rangle^{-2} \lambda J\right)_{y^{\prime}=x^{\prime}} \in R, \quad|\alpha| \geq 1 .
$$


We now study $m \bar{\Lambda} J$ (or $\lambda \bar{\Lambda} J)$. Since

$$
\begin{aligned}
\partial_{\xi^{\prime}}^{\alpha^{\prime \prime}} \partial_{y^{\prime}}^{\alpha}(m J)_{y^{\prime}=x^{\prime}} \in \mu S & \left(\left(r^{-1} w^{1 / 2}\left\langle\mu \xi^{\prime}\right\rangle^{\kappa}+r^{-1}\left\langle\mu \xi^{\prime}\right\rangle^{-1 / 2}\right.\right. \\
& \left.\left.+\left\langle\mu \xi^{\prime}\right\rangle^{\kappa^{\prime}}\right)\left\langle\xi^{\prime}\right\rangle_{\mu}^{|\alpha| / 2-3\left|\alpha^{\prime \prime}\right| / 4}, g\right),
\end{aligned}
$$

hence $\partial_{\xi^{\prime}}^{\alpha^{\prime}} \bar{\Lambda} \partial_{\xi^{\prime}}^{\alpha^{\prime \prime}} \partial_{y^{\prime}}^{\alpha}(m J)_{y^{\prime}=x^{\prime}} \in R$ if $\left|\alpha^{\prime}\right| \geq 1$ where $\alpha^{\prime}+\alpha^{\prime \prime}=\alpha$. This shows

$$
\partial_{\xi^{\prime}}^{\alpha} \partial_{y^{\prime}}^{\alpha}(\bar{\Lambda} m J)_{y^{\prime}=x^{\prime}}=C_{1} \bar{\Lambda}+R, \quad|\alpha| \geq 1 .
$$

Thus we have

$$
\partial_{\xi^{\prime}}^{\alpha} \partial_{y^{\prime}}^{\alpha}(\tilde{M} \tilde{\Lambda} J)_{y^{\prime}=x^{\prime}}=\partial_{\xi^{\prime}}^{\alpha} \partial_{y^{\prime}}^{\alpha}(\bar{M} \bar{\Lambda} J)_{y^{\prime}=x^{\prime}}+C_{1} \tilde{\Lambda}\left(x, x^{\prime}, \xi\right)+R
$$

because $\left(C_{1} \lambda\right)_{y^{\prime}=x^{\prime}} \in R$. Finally, we consider $\partial_{\xi^{\prime}}^{\alpha} \partial_{y^{\prime}}^{\alpha}(\bar{M} \bar{\Lambda} J)$. Let $\left|\alpha^{\prime}\right| \geq 1$. Then from (5.5) one sees that

$$
\partial_{\xi^{\prime}}^{\alpha^{\prime}}(\bar{M} \bar{\Lambda})\left(\partial_{\xi^{\prime}}^{\alpha^{\prime \prime}} \partial_{y^{\prime}}^{\alpha} J\right)_{y^{\prime}=x^{\prime}}=C_{1} \bar{\Lambda}+R
$$

because $\bar{M}=\bar{\Lambda}+S\left(w^{3 / 2}\left\langle\mu \xi^{\prime}\right\rangle, w^{-1} g_{0}\right)$ and

$$
\partial_{\xi_{1}} \bar{\Lambda}, \quad \partial_{\xi_{1}} \bar{M}=\mu S(r, g)+S\left(\left\langle\mu \xi^{\prime}\right\rangle^{\kappa^{\prime}}\left\langle\xi^{\prime}\right\rangle_{\mu}^{-1}, g\right)
$$

thanks to (4.7). Thus we conclude that

$$
\left(\partial_{\xi^{\prime}}^{\alpha} \partial_{y^{\prime}}^{\alpha} \bar{M} \bar{\Lambda} J\right)_{y^{\prime}=x^{\prime}}=\bar{M} \bar{\Lambda}\left(\partial_{\xi^{\prime}}^{\alpha} \partial_{y^{\prime}}^{\alpha} J\right)_{y^{\prime}=x^{\prime}}+C_{1} \bar{\Lambda}+R .
$$

Noting that

$$
\bar{M} \bar{\Lambda}\left(\partial_{\xi^{\prime}}^{\alpha} \partial_{y^{\prime}}^{\alpha} J\right)_{y^{\prime}=x^{\prime}}=\tilde{M} \tilde{\Lambda}\left(\partial_{\xi^{\prime}}^{\alpha} \partial_{y^{\prime}}^{\alpha} J\right)_{y^{\prime}=x^{\prime}}+C_{1} \tilde{\Lambda}\left(x, x^{\prime}, \xi\right)+R,
$$

we have the desired assertion.

We next consider $\tilde{\phi}_{1} \tilde{\Lambda} J$. From Proposition 5.2 we can write

$$
\tilde{\phi}_{1}\left(x, y^{\prime}, \xi^{\prime}\right)=\tilde{\phi}_{1}\left(x, \xi^{\prime}\right)+\nu_{1}\left(x, y^{\prime}, \xi^{\prime}\right)
$$

with $\nu_{1}\left(x, y^{\prime}, \xi\right) \in \mu S^{\#}\left(\left(r^{-1}\left\langle\mu \xi^{\prime}\right\rangle^{\kappa}+\left\langle\mu \xi^{\prime}\right\rangle^{\kappa^{\prime}}\right), g\right)$. Let $\tilde{\phi}_{1} \tilde{\Lambda} J=\phi_{1} \bar{\Lambda} J+\bar{\Lambda} \nu_{1} J+$ $\phi_{1} \lambda J+\nu_{1} \lambda J$. It is easy to check that $\partial_{\xi^{\prime}}^{\alpha} \partial_{y^{\prime}}^{\alpha}\left(\nu_{1} \lambda J\right)_{y^{\prime}=x^{\prime}} \in R$ for $|\alpha| \geq 1$. Noting that

$$
\phi_{1}\left(x, \xi^{\prime}\right) \in S\left(w^{1 / 2}\left\langle\mu \xi^{\prime}\right\rangle, g\right) \cap S\left(\left\langle\mu \xi^{\prime}\right\rangle, g_{0}\right),
$$

we have $\partial_{\xi^{\prime}}^{\alpha} \partial_{y^{\prime}}^{\alpha}\left(\phi_{1} \lambda J\right)_{y^{\prime}=x^{\prime}} \in R,|\alpha| \geq 1$. Let $\left|\alpha^{\prime}\right| \geq 1$; then from (5.6) we have

$$
\partial_{\xi^{\prime}}^{\alpha^{\prime}} \bar{\Lambda} \partial_{\xi^{\prime}}^{\alpha^{\prime \prime}} \partial_{y^{\prime}}^{\alpha}\left(\nu_{1} J\right)_{y^{\prime}=x^{\prime}} \in R, \quad\left|\alpha^{\prime}\right| \geq 1 .
$$

Since $\partial_{\xi^{\prime}}^{\alpha} \partial_{y^{\prime}}^{\alpha}\left(\nu_{1} J\right)_{y^{\prime}=x^{\prime}} \in \mu^{7 / 4} S\left(r^{-2}\left\langle\mu \xi^{\prime}\right\rangle^{\kappa-3 / 4}, g\right)+\mu S\left(\left\langle\mu \xi^{\prime}\right\rangle^{\kappa^{\prime}}, g\right)$ and noting that $S\left(r^{-2}\left\langle\mu \xi^{\prime}\right\rangle^{\kappa-3 / 4}, g\right) \subset \mu^{-1 / 2} S\left(r^{-2} w\left\langle\mu \xi^{\prime}\right\rangle^{\kappa-1 / 4}, g\right)$, we conclude that

$$
\partial_{\xi^{\prime}}^{\alpha} \partial_{y^{\prime}}^{\alpha}\left(\bar{\Lambda} \nu_{1} J\right)_{y^{\prime}=x^{\prime}}=C_{1}\left(x, \xi^{\prime}\right) \bar{\Lambda}+C_{2}\left(x, \xi^{\prime}\right) \bar{\Lambda}+R, \quad|\alpha| \geq 1 .
$$

Thus we can write

$$
\left[\partial_{\xi^{\prime}}^{\alpha} \partial_{y^{\prime}}^{\alpha}\left(\tilde{\phi}_{1} \tilde{\Lambda} J\right)\right]_{y^{\prime}=x^{\prime}}=\left[\partial_{\xi^{\prime}}^{\alpha} \partial_{y^{\prime}}^{\alpha}\left(\phi_{1} \bar{\Lambda} J\right)\right]_{y^{\prime}=x^{\prime}}+C_{1} \bar{\Lambda}+C_{2} \bar{\Lambda}+R
$$

We check $\partial_{\xi^{\prime}}^{\alpha} \partial_{y^{\prime}}^{\alpha}\left(\phi_{1} \bar{\Lambda} J\right)$. Let $\left|\alpha^{\prime}\right| \geq 1$ and $|\alpha| \geq 2$. Then from (5.7) and (5.5) it follows that

$$
\partial_{\xi^{\prime}}^{\alpha^{\prime}}\left(\phi_{1} \bar{\Lambda}\right)\left(\partial_{\xi^{\prime}}^{\alpha^{\prime \prime}} \partial_{y^{\prime}}^{\alpha} J\right)_{y^{\prime}=x^{\prime}}=C_{1} \bar{\Lambda}+R
$$


Let $\left|\alpha^{\prime}\right|=|\alpha|=1$, and consider $\bar{\Lambda} \partial_{\xi^{\prime}}^{\alpha} \phi_{1}\left(\partial_{y^{\prime}}^{\alpha} J\right)_{y^{\prime}=x^{\prime}}$ and $\phi_{1}\left(\partial_{\xi^{\prime}}^{\alpha} \bar{\Lambda}\right)\left(\partial_{y^{\prime}}^{\alpha} J\right)_{y^{\prime}=x^{\prime}}$. From (5.6) and (5.7), taking (5.5) into account again we see that

$$
\phi_{1}\left(\partial_{\xi^{\prime}}^{\alpha} \bar{\Lambda}\right)\left(\partial_{y^{\prime}}^{\alpha} J\right)_{y^{\prime}=x^{\prime}} \in R, \quad \partial_{\xi^{\prime}}^{\alpha} \phi_{1}\left(\partial_{y^{\prime}}^{\alpha} J\right)_{y^{\prime}=x^{\prime}} \in C_{1}+C_{2} .
$$

Hence we conclude that

$$
\partial_{\xi^{\prime}}^{\alpha} \partial_{y^{\prime}}^{\alpha}\left(\phi_{1} \bar{\Lambda} J\right)_{y^{\prime}=x^{\prime}}=\phi_{1} \bar{\Lambda}\left(\partial_{\xi^{\prime}}^{\alpha} \partial_{y^{\prime}}^{\alpha} J\right)_{y^{\prime}=x^{\prime}}+C_{1}\left(x, \xi^{\prime}\right) \bar{\Lambda}+C_{2}\left(x, \xi^{\prime}\right) \bar{\Lambda}+R
$$

Noting that $\left(C_{2} \lambda\right)_{y^{\prime}=x^{\prime}} \in R$ and

$$
\begin{aligned}
\phi_{1} \bar{\Lambda}\left(\partial_{\xi^{\prime}}^{\alpha} \partial_{y^{\prime}}^{\alpha} J\right)_{y^{\prime}=x^{\prime}} & =\phi_{1} \tilde{\Lambda}\left(x, x^{\prime}, \xi\right)\left(\partial_{\xi^{\prime}}^{\alpha} \partial_{y^{\prime}}^{\alpha} J\right)_{y^{\prime}=x^{\prime}}+R \\
& =\left[\tilde{\phi}_{1} \tilde{\Lambda}\left(\partial_{\xi^{\prime}}^{\alpha} \partial_{y^{\prime}}^{\alpha} J\right)\right]_{y^{\prime}=x^{\prime}}-\left[\nu_{1} \tilde{\Lambda}\left(\partial_{\xi^{\prime}}^{\alpha} \partial_{y^{\prime}}^{\alpha} J\right)\right]_{y^{\prime}=x^{\prime}}+R
\end{aligned}
$$

we get the second assertion for $\left(\nu_{1} \partial_{\xi^{\prime}}^{\alpha} \partial_{y^{\prime}}^{\alpha} J\right)_{y^{\prime}=x^{\prime}} \in C_{1}+C_{2}$.

We turn to considering $\tilde{\phi}_{2}^{2} J$ and $\tilde{\theta}^{2} J$. From Proposition 5.2 one can write

$$
\begin{gathered}
\tilde{\phi}_{2}\left(x, y^{\prime}, \xi^{\prime}\right)=\phi_{2}\left(x, \xi^{\prime}\right)+\nu_{2}\left(x, y^{\prime}, \xi^{\prime}\right), \\
\tilde{\theta}\left(x, y^{\prime}, \xi^{\prime}\right)=\theta\left(x, \xi^{\prime}\right)+\nu_{3}\left(x, y^{\prime}, \xi^{\prime}\right)
\end{gathered}
$$

with $\nu_{i}\left(x, y^{\prime}, \xi^{\prime}\right) \in \mu S^{\#}\left(\left(r^{-1} w^{1 / 2}\left\langle\mu \xi^{\prime}\right\rangle^{\kappa}+\left\langle\mu \xi^{\prime}\right\rangle^{\kappa^{\prime}}\right), g\right)$. Since $\phi_{2}\left(x, \xi^{\prime}\right) \in S\left(r\left\langle\mu \xi^{\prime}\right\rangle\right.$, $g$ ), writing $\tilde{\phi}\left(x, y^{\prime}, \xi^{\prime}\right)^{2}=\phi_{2}\left(x, \xi^{\prime}\right)^{2}+r\left(x, y^{\prime}, \xi^{\prime}\right)$ it is clear that $\partial_{\xi^{\prime}}^{\alpha} \partial_{y^{\prime}}^{\alpha}(r J)_{y^{\prime}=x^{\prime}} \in R$ for $|\alpha| \geq 1$. Recalling (5.5) and $\partial_{\xi^{\prime}}^{\alpha^{\prime}} \phi_{2}^{2} \in S\left(r^{2}\langle\mu \xi\rangle^{2}\left\langle\xi^{\prime}\right\rangle_{\mu}^{-\left|\alpha^{\prime}\right|}, g\right)$, we have

$$
\partial_{\xi^{\prime}}^{\alpha^{\prime}} \phi_{2}^{2}\left(\partial_{\xi^{\prime}}^{\alpha^{\prime \prime}} \partial_{y^{\prime}}^{\alpha} J\right)_{y^{\prime}=x^{\prime}} \in R
$$

if $\left|\alpha^{\prime}\right| \geq 1$. Thus we get

$$
\partial_{\xi^{\prime}}^{\alpha} \partial_{y^{\prime}}^{\alpha}\left(\tilde{\phi}_{2}^{2} J\right)_{y^{\prime}=x^{\prime}}=\phi_{2}^{2}\left(\partial_{\xi^{\prime}}^{\alpha} \partial_{y^{\prime}}^{\alpha} J\right)_{y^{\prime}=x^{\prime}}+R
$$

Since $\left(r \partial_{\xi^{\prime}}^{\alpha} \partial_{y^{\prime}}^{\alpha} J\right)_{y^{\prime}=x^{\prime}} \in R,|\alpha| \geq 1$, we have the third assertion. Since

$$
\theta\left(x, \xi^{\prime}\right) \in S\left(w\left\langle\mu \xi^{\prime}\right\rangle, g\right) \cap S\left(\left\langle\mu \xi^{\prime}\right\rangle, g_{0}\right),
$$

then writing $\tilde{\theta}\left(x, y^{\prime}, \xi^{\prime}\right)^{2}=\theta\left(x, \xi^{\prime}\right)^{2}+r\left(x, y^{\prime}, \xi^{\prime}\right)$, it is easy to see

$$
\left(\partial_{\xi^{\prime}}^{\alpha} \partial_{y^{\prime}}^{\alpha} r J\right)_{y^{\prime}=x^{\prime}},\left(r \partial_{\xi^{\prime}}^{\alpha} \partial_{y^{\prime}}^{\alpha} J\right)_{y^{\prime}=x^{\prime}} \in R, \quad|\alpha| \geq 1 .
$$

Since $\partial_{\xi^{\prime}}^{\alpha^{\prime}} \theta\left(x, \xi^{\prime}\right)^{2} \in S\left(w^{\left(4-\left|\alpha^{\prime}\right|\right) / 2}\left\langle\mu \xi^{\prime}\right\rangle^{2}\left\langle\xi^{\prime}\right\rangle_{\mu}^{-\left|\alpha^{\prime}\right|}, g\right)$ for $\left|\alpha^{\prime}\right| \leq 4$, it follows from (5.5) that $\partial_{\xi^{\prime}}^{\alpha^{\prime}} \theta^{2}\left(\partial_{\xi^{\prime}}^{\alpha^{\prime \prime}} \partial_{y^{\prime}}^{\alpha} J\right)_{y^{\prime}=x^{\prime}} \in R$ if $\left|\alpha^{\prime}\right| \geq 1$, and hence we have, for $|\alpha| \geq 1$,

$$
\left[\partial_{\xi^{\prime}}^{\alpha} \partial_{y^{\prime}}^{\alpha}\left(\tilde{\theta}^{2} J\right)\right]_{y^{\prime}=x^{\prime}}=\theta\left(x, \xi^{\prime}\right)^{2}\left(\partial_{\xi^{\prime}}^{\alpha} \partial_{y^{\prime}}^{\alpha} J\right)_{y^{\prime}=x^{\prime}}+R .
$$

Since $\left[\tilde{\theta}^{2}\left(\partial_{\xi^{\prime}}^{\alpha} \partial_{y^{\prime}}^{\alpha} J\right)\right]_{y^{\prime}=x^{\prime}}=\theta\left(x, \xi^{\prime}\right)^{2}\left(\partial_{\xi^{\prime}}^{\alpha} \partial_{y^{\prime}}^{\alpha} J\right)_{y^{\prime}=x^{\prime}}+R$, we get the fourth assertion.

\section{Proof of Proposition 5.3}

We note that

$$
j\left(x, \xi^{\prime}\right)=1+S\left(\left(r^{-2} w+r^{-1} w^{1 / 2}\right)\left\langle\mu \xi^{\prime}\right\rangle^{\kappa}\left\langle\xi^{\prime}\right\rangle_{\mu}^{-3 / 4}, g\right),
$$

and hence

$\partial_{\xi^{\prime}}^{\beta} \partial_{x^{\prime}}^{\alpha} j \in S\left(\left(r^{-1}+\left\langle\xi^{\prime}\right\rangle_{\mu}^{1 / 4}\right)^{1+\alpha_{1}}\left\langle\mu \xi^{\prime}\right\rangle^{\kappa}\left\langle\xi^{\prime}\right\rangle_{\mu}^{-3 / 4+\left(|\alpha|-\alpha_{1}\right) / 4-3|\beta| / 4}, g\right), \quad|\alpha+\beta| \geq 1$. 
Then using similar easier arguments as in the proof of Lemma 5.5, we can show that with $\tilde{\Lambda}=\tilde{\Lambda}\left(x, x^{\prime}, \xi\right), \tilde{M}=\tilde{M}\left(x, x^{\prime}, \xi\right)$,

$$
\tilde{\Lambda} \tilde{M} \sum_{|\alpha|<5} \frac{1}{\alpha !}\left(D_{y^{\prime}}^{\alpha} \partial_{\xi^{\prime}}^{\alpha} J\right)_{y^{\prime}=x^{\prime}}=\tilde{\Lambda} \tilde{M} \# j+C_{1} \tilde{\Lambda}+R .
$$

The proof for the other cases is similar.

Since we can write $\operatorname{Op}^{0}\left(\mu^{5 / 4} S\left(\left\langle\mu \xi^{\prime}\right\rangle^{-1}, \bar{g}\right) \xi_{0}^{2}\right)=\tilde{\Lambda} \tilde{M} \# j^{\prime}+C_{1} \tilde{\Lambda}+R$ with $j^{\prime} \in$ $\mu^{5 / 4} S\left(\left\langle\mu \xi^{\prime}\right\rangle^{-1}, \bar{g}\right)$, combining Propositions 5.1 and 5.3 we get

$$
\begin{aligned}
& \operatorname{Op}^{0}\left(e^{\tilde{\phi}}\right) \operatorname{Op}^{0}(p) \operatorname{Op}^{1}\left(e^{-\tilde{\phi}}\right) \\
& \quad=\operatorname{Op}^{0}\left(\tilde{p}(x, \xi) \# \tilde{j}+C_{1} \tilde{\Lambda}\left(x, x^{\prime}, \xi\right)+C_{2} \tilde{\Lambda}\left(x, x^{\prime}, \xi\right)+\bar{R}\right),
\end{aligned}
$$

where $\tilde{j}=j+j^{\prime}$, and hence $\operatorname{Op}^{0}\left(e^{\tilde{\phi}}\right) \mathrm{Op}^{1}\left(e^{-\tilde{\phi}}\right)=\mathrm{Op}^{0}(\tilde{j})$ and

$$
\begin{aligned}
\tilde{p} & =-\tilde{M}\left(x, x^{\prime}, \xi\right) \tilde{\Lambda}\left(x, x^{\prime}, \xi\right)+2 \tilde{\phi}_{1}\left(x, x^{\prime}, \xi^{\prime}\right) \tilde{\Lambda}\left(x, x^{\prime}, \xi\right)+\tilde{Q}\left(x, x^{\prime}, \xi^{\prime}\right), \\
\tilde{Q}\left(x, x^{\prime}, \xi^{\prime}\right) & =\tilde{\phi}_{2}\left(x, x^{\prime}, \xi^{\prime}\right)^{2}+\tilde{\theta}\left(x, x^{\prime}, \xi^{\prime}\right)^{2}, \\
C_{1}\left(x, \xi^{\prime}\right) & \in \mu S\left(\left\langle\mu \xi^{\prime}\right\rangle^{\kappa^{\prime}}, g\right)+\mu S(1, \bar{g}), \\
C_{2}\left(x, \xi^{\prime}\right) & \in \mu^{5 / 4} S\left(r^{-2} w\left\langle\mu \xi^{\prime}\right\rangle^{\kappa-1 / 4}, g\right),
\end{aligned}
$$

where $\bar{R}$ denotes the symbol class

$$
\mu S\left(r\left\langle\mu \xi^{\prime}\right\rangle^{\kappa^{\prime}+1}, g\right)+\mu S\left(\left\langle\mu \xi^{\prime}\right\rangle, \bar{g}\right)
$$

or, rather, a symbol itself belonging to $\bar{R}$. Thanks to Lemma 5.4 and Proposition 5.2 , one can write

$$
\tilde{\phi}_{2}=\phi_{2}\left(x, \xi^{\prime}\right)+i h\left(x, \xi^{\prime}\right)+\mu S\left(\left(r^{-1}\left\langle\mu \xi^{\prime}\right\rangle^{\kappa-1 / 4}+\left\langle\mu \xi^{\prime}\right\rangle^{\kappa^{\prime}}\right), g\right)
$$

with $h \in \mu S\left(r^{-1} w^{1 / 2}\left\langle\mu \xi^{\prime}\right\rangle^{\kappa}, g\right)$ which is real; then we see that

$$
\left\{\begin{array}{l}
\operatorname{Re} \tilde{\phi}_{2}\left(x, x^{\prime}, \xi^{\prime}\right)^{2}=\phi_{2}\left(x, \xi^{\prime}\right)^{2}+\bar{R}, \\
\operatorname{Im} \tilde{\phi}_{2}\left(x, x^{\prime}, \xi^{\prime}\right)^{2}=\mu S\left(w^{1 / 2}\left\langle\mu \xi^{\prime}\right\rangle^{1+\kappa}, g\right)+\bar{R} .
\end{array}\right.
$$

From Proposition 5.2 we can write

$$
\tilde{\theta}\left(x, x^{\prime}, \xi^{\prime}\right)=\theta\left(x, \xi^{\prime}\right)+i f+\mu^{5 / 4} S\left(r^{-1} w^{1 / 2}\left\langle\mu \xi^{\prime}\right\rangle^{2 \kappa-1 / 4}, g\right)+\mu S\left(\left\langle\mu \xi^{\prime}\right\rangle^{\kappa^{\prime}}, g\right)
$$

with $f \in \mu S\left(r^{-1} w^{1 / 2}\left\langle\mu \xi^{\prime}\right\rangle^{\kappa}, g\right)$ which is real. Noting

$$
S\left(w^{1 / 2}\left\langle\mu \xi^{\prime}\right\rangle^{2 \kappa+3 / 4}, g\right) \subset \mu^{-3 / 4} S\left(w^{2}\left\langle\mu \xi^{\prime}\right\rangle^{2}, g\right)
$$

and (5.8), we obtain (because $w \leq r$ )

$$
\begin{aligned}
& \operatorname{Re} \tilde{\theta}\left(x, x^{\prime}, \xi^{\prime}\right)^{2}=\theta\left(x, \xi^{\prime}\right)^{2}+\mu^{1 / 2} S\left(w^{2}\left\langle\mu \xi^{\prime}\right\rangle^{2}, g\right)+\bar{R}, \\
& \operatorname{Im} \tilde{\theta}\left(x, x^{\prime}, \xi^{\prime}\right)^{2}=\mu S\left(w^{1 / 2}\left\langle\mu \xi^{\prime}\right\rangle^{\kappa+1}, g\right)+\bar{R} .
\end{aligned}
$$

Since $w^{2}\left\langle\mu \xi^{\prime}\right\rangle^{2}=\left\langle\mu \xi^{\prime}\right\rangle^{-2} \phi_{1}^{4}+\mu\left\langle\mu \xi^{\prime}\right\rangle$, with $\alpha\left(x, \xi^{\prime}\right) \in S(1, g)$ such that $C^{-2} \leq \alpha \leq$ $C$ we can write

$$
\left\{\begin{array}{l}
\operatorname{Re} \tilde{\theta}^{2}=\alpha\left(x, \xi^{\prime}\right) \theta\left(x, \xi^{\prime}\right)^{2}+\bar{R}, \\
\operatorname{Im} \tilde{\theta}^{2}=\mu S\left(w^{1 / 2}\left\langle\mu \xi^{\prime}\right\rangle^{\kappa+1}, g\right)+\bar{R} .
\end{array}\right.
$$


From (5.9) and (5.10) we have

$$
\begin{aligned}
& \operatorname{Re} \tilde{Q}\left(x, x^{\prime}, \xi^{\prime}\right)=\phi_{2}^{2}+\alpha \theta^{2}+\bar{R}, \\
& \operatorname{Im} \tilde{Q}\left(x, x^{\prime}, \xi^{\prime}\right)=\mu S\left(w^{1 / 2}\left\langle\mu \xi^{\prime}\right\rangle^{1+\kappa}, g\right)+\bar{R} .
\end{aligned}
$$

Let us write $\tilde{j}=1+\mu^{1 / 4} S(1, \bar{g})$. For small $\mu$ there exists an inverse of $\operatorname{Op}^{0}(\tilde{j})$ in $L^{2}$ which is actually given by $\operatorname{Op}^{0}\left(\tilde{j}^{-1}\right)$ with a $\tilde{j}^{-1} \in S(1, \bar{g})$ (see [1]) so that

$$
\mathrm{Op}^{0}(\tilde{j}) \mathrm{Op}^{0}\left(\tilde{j}^{-1}\right)=I \text {. }
$$

Let $C_{i}\left(x, \xi^{\prime}\right)$ be as above. Then it is easy to check that

$$
\begin{aligned}
& \left(C_{1} \tilde{\Lambda}\right) \# \tilde{j}^{-1}=C_{1}\left(x, \xi^{\prime}\right) \tilde{\Lambda}\left(x, x^{\prime}, \xi\right)+\tilde{C}_{1} \tilde{\Lambda}+\mu S\left(\left\langle\mu \xi^{\prime}\right\rangle, \bar{g}\right), \\
& \left(C_{2} \tilde{\Lambda}\right) \# \tilde{j}^{-1}=C_{2}\left(x, \xi^{\prime}\right) \tilde{\Lambda}\left(x, x^{\prime}, \xi^{\prime}\right)+\tilde{C}_{1} \tilde{\Lambda}+\mu S\left(\left\langle\mu \xi^{\prime}\right\rangle, \bar{g}\right),
\end{aligned}
$$

where $\tilde{C}_{1}\left(x, \xi^{\prime}\right) \in \mu S\left(\left\langle\mu \xi^{\prime}\right\rangle^{\kappa^{\prime}}, g\right)$. With a constant $K>0$, let us put

$$
\omega=2 r^{-2} w\left\langle\mu \xi^{\prime}\right\rangle^{\kappa}\left\{\phi_{1}, \psi\right\}+\mu K\left\langle\mu \xi^{\prime}\right\rangle^{\kappa} .
$$

Since $\left\{\phi_{1}, \psi\right\} \geq c_{1} \mu$ if $r$ is small, then taking $K$ large it is clear that

$$
\omega \geq c \mu r^{-2} w\left\langle\mu \xi^{\prime}\right\rangle^{\kappa}
$$

with some $c>0$. Finally, we can conclude the following.

PROPOSITION 5.4

We have

$$
\mathrm{Op}^{0}\left(e^{\tilde{\phi}}\right) \mathrm{Op}^{0}(p) \mathrm{Op}^{1}\left(e^{-\tilde{\phi}}\right) \mathrm{Op}^{0}\left(\tilde{j}^{-1}\right)=\mathrm{Op}^{0}(\hat{p})
$$

with

$$
\begin{aligned}
\hat{p}= & -\left(M-i\left\langle\mu \xi^{\prime}\right\rangle^{\kappa^{\prime}}+\tilde{m}\right)\left(\Lambda-i\left\langle\mu \xi^{\prime}\right\rangle^{\kappa^{\prime}}+\tilde{\lambda}\right) \\
& +2\left(\phi_{1}+i \omega+C\right)\left(\Lambda-i\left\langle\mu \xi^{\prime}\right\rangle^{\kappa^{\prime}}+\tilde{\lambda}\right) \\
& +Q+\mu S\left(r\left\langle\mu \xi^{\prime}\right\rangle^{1+\kappa^{\prime}}, g\right)+\mu S\left(\left\langle\mu \xi^{\prime}\right\rangle, \bar{g}\right),
\end{aligned}
$$

where $\tilde{m}\left(x, \xi^{\prime}\right), \tilde{\lambda}\left(x, \xi^{\prime}\right) \in \mu S\left(\left\langle\mu \xi^{\prime}\right\rangle^{\kappa^{\prime}}, g\right)+\sqrt{\mu} S(1, g), \omega \in \mu S\left(r^{-2} w\left\langle\mu \xi^{\prime}\right\rangle^{\kappa}, g\right)$ which is real, $C\left(x, \xi^{\prime}\right) \in \mu^{5 / 4} S\left(r^{-2} w\left\langle\mu \xi^{\prime}\right\rangle^{\kappa}, g\right)$, and

$$
\begin{aligned}
& Q=\phi_{2}^{2}+\alpha \theta^{2}+i Q_{1}, \quad Q_{1} \in \mu S\left(w^{1 / 2}\left\langle\mu \xi^{\prime}\right\rangle^{1+\kappa}, g\right), Q_{1} \text { is real, } \\
& \Lambda=\xi_{0}+\lambda_{1}-k\left\langle\mu \xi^{\prime}\right\rangle^{-2} \phi_{1}^{3}, \quad M=\xi_{0}+\lambda_{1}+k\left\langle\mu \xi^{\prime}\right\rangle^{-2} \phi_{1}^{3} .
\end{aligned}
$$

Moreover, we have $\omega \geq c \mu r^{-2} w\left\langle\mu \xi^{\prime}\right\rangle^{\kappa}$ with some $c>0$ and

$$
\left\{\Lambda, \phi_{2}\right\} \in \mu S\left(r\left\langle\mu \xi^{\prime}\right\rangle, g\right), \quad\left\{\Lambda, \phi_{1}\right\} \in \mu S\left(\left(r+w^{1 / 2}\right)\left\langle\mu \xi^{\prime}\right\rangle, g\right) .
$$

Proof

From Proposition 5.2 we have

$$
\begin{gathered}
\tilde{M}\left(x, x^{\prime}, \xi\right)=M(x, \xi)-i\left\langle\mu \xi^{\prime}\right\rangle^{\kappa^{\prime}}+\tilde{m}, \\
\tilde{\Lambda}\left(x, x^{\prime}, \xi\right)=\Lambda(x, \xi)-i\left\langle\mu \xi^{\prime}\right\rangle^{\kappa^{\prime}}+\tilde{\lambda}
\end{gathered}
$$


where $\tilde{\lambda}, \tilde{m} \in \mu S\left(\left\langle\mu \xi^{\prime}\right\rangle^{\kappa^{\prime}}, g\right)+\sqrt{\mu} S(1, g)$. From Proposition 5.2 and (5.11) we can write

$$
\tilde{\phi}_{1}\left(x, x^{\prime}, \xi^{\prime}\right) \tilde{\Lambda}\left(x, x^{\prime}, \xi\right)=\left(\phi_{1}+i \omega+C+\mu S\left(\left\langle\mu \xi^{\prime}\right\rangle^{\kappa^{\prime}}, g\right)\right) \tilde{\Lambda}\left(x, x^{\prime}, \xi\right)
$$

with $C \in \mu^{5 / 4}\left(r^{-2} w\left\langle\mu \xi^{\prime}\right\rangle^{\kappa}, g\right)$, where we move the term $\mu S\left(\left\langle\mu \xi^{\prime}\right\rangle^{\kappa^{\prime}}, g\right)$ into $\tilde{m}$, which completes the proof.

\section{Energy estimate (proof of Theorem 1.1)}

Let $P$ be the operator with symbol given in Section 4 , that is, the symbol which is equal, in a conic neighborhood of the reference point, to that given by the right-hand side of (2.2).

In this section we derive a priori estimates for the transformed operator $\tilde{P}$,

$$
\tilde{P}=\mathrm{Op}^{0}\left(e^{\tilde{\phi}}\right) P \mathrm{Op}^{1}\left(e^{-\tilde{\phi}}\right) \mathrm{Op}^{0}\left(\tilde{j}^{-1}\right),
$$

where the principal symbol of $\tilde{P}$ is given in Proposition 5.4. Let us denote $\mathrm{Op}^{1 / 2}(a)=a^{w}$, the Weyl quantization of $a$. By the same $M, \Lambda$, we denote $M=$ $D_{0}-i\left\langle\mu D^{\prime}\right\rangle^{\kappa^{\prime}}-m, \Lambda=D_{0}-i\left\langle\mu D^{\prime}\right\rangle^{\kappa^{\prime}}-\lambda$, where $\lambda=-\left(\lambda_{1}-k\left\langle\mu \xi^{\prime}\right\rangle^{-2} \phi_{1}^{3}+\tilde{\lambda}\right)^{w}$, $m=-\left(\lambda_{1}+k\left\langle\mu \xi^{\prime}\right\rangle^{-2} \phi_{1}^{3}+\tilde{m}\right)^{w}$. We also denote $\left(Q+2 \mu\left\langle\mu \xi^{\prime}\right\rangle\right)^{w}$ by the same $Q$ and $2\left(\phi_{1}+i \omega+C\right)^{w}$ by $B$. Note that one can write

$$
\tilde{P}=-M \Lambda+B \Lambda+Q+\tilde{P}_{1}
$$

with $\tilde{P}_{1}=a^{w} \Lambda+b^{w}, a \in S(1, \bar{g}), b \in \bar{R}$. Recall the following.

PROPOSITION 6.1 ([3, PROPOSITION 4.1])

We have

$$
\begin{aligned}
2 \operatorname{Im}\left(\left(\tilde{P}-\tilde{P}_{1}\right) v, \Lambda v\right)= & \frac{d}{d x_{0}}\left(\|\Lambda v\|^{2}+((\operatorname{Re} Q) v, v)\right) \\
& +2\left\|\left\langle\mu D^{\prime}\right\rangle^{\kappa^{\prime} / 2} \Lambda v\right\|^{2}+2 \operatorname{Re}\left(\left\langle\mu D^{\prime}\right\rangle^{\kappa^{\prime}}(\operatorname{Re} Q) v, v\right) \\
& +2((\operatorname{Im} B) \Lambda v, \Lambda v)+2((\operatorname{Im} m) \Lambda v, \Lambda v)+2 \operatorname{Re}(\Lambda v,(\operatorname{Im} Q) v) \\
& +\operatorname{Im}\left(\left[D_{0}-\operatorname{Re} \lambda, \operatorname{Re} Q\right] v, v\right)+2 \operatorname{Re}((\operatorname{Re} Q) v,(\operatorname{Im} \lambda) v)
\end{aligned}
$$

for any $v \in C^{2}\left((-T,-T) ; \mathcal{S}\left(\mathbb{R}^{n}\right)\right)$.

In this energy identity, the main term that controls (any) lower-order term is

$$
((\operatorname{Im} B) \Lambda v, \Lambda v)+\operatorname{Re}\left(\left\langle\mu D^{\prime}\right\rangle^{\kappa^{\prime}}(\operatorname{Re} Q) v, v\right) .
$$

We first consider $((\operatorname{Im} B) \Lambda v, \Lambda v)$. Since $\operatorname{Im} C \in \mu^{5 / 4} S\left(r^{-2} w\left\langle\mu \xi^{\prime}\right\rangle^{\kappa}, g\right)$, hence one can write

$$
\omega+\operatorname{Im} C=\mu\left(\sqrt{w} r^{-1}\left\langle\mu \xi^{\prime}\right\rangle^{\kappa / 2} a\right) \#\left(\sqrt{w} r^{-1}\left\langle\mu \xi^{\prime}\right\rangle^{\kappa / 2} a\right)+\mu S\left(\left\langle\mu \xi^{\prime}\right\rangle^{\kappa}, g\right)
$$

with $a=\sqrt{\mu^{-1} w^{-1} r^{2}\left\langle\mu \xi^{\prime}\right\rangle^{-\kappa}(\omega+\operatorname{Im} C)} \in S(1, g)$, where $a \geq c>0$. This shows that

$$
((\operatorname{Im} B) \Lambda v, \Lambda v) \geq \mu\left\|\left(\sqrt{w} r^{-1}\left\langle\mu \xi^{\prime}\right\rangle^{\kappa / 2} a\right)^{w} \Lambda v\right\|^{2}-C \mu\left\|\left\langle\mu D^{\prime}\right\rangle^{\kappa^{\prime} / 2} \Lambda v\right\|^{2}
$$


We next study the terms $((\operatorname{Re} Q) v, v)$ and $\operatorname{Re}\left(\left\langle\mu D^{\prime}\right\rangle^{\kappa^{\prime}}(\operatorname{Re} Q) v, v\right)$. Note that $\left(\left\langle\xi^{\prime}\right\rangle_{\mu}^{-1}\left\langle\mu \xi^{\prime}\right\rangle^{2}=\mu\left\langle\mu \xi^{\prime}\right\rangle\right)$

$$
\begin{aligned}
\operatorname{Re} Q & =q \# q+\mu\left\langle\mu \xi^{\prime}\right\rangle+\mu S\left(r\left\langle\mu \xi^{\prime}\right\rangle, g\right)+\mu^{3 / 2} S\left(\left\langle\mu \xi^{\prime}\right\rangle, g\right), \\
q & =\sqrt{\phi_{2}^{2}+\alpha \theta^{2}+\mu\left\langle\mu \xi^{\prime}\right\rangle} \in S\left(r\left\langle\mu \xi^{\prime}\right\rangle, g\right) .
\end{aligned}
$$

Since $q \geq c\left\langle\mu \xi^{\prime}\right\rangle r$ with some $c>0$,

$$
((\operatorname{Re} Q) v, v) \geq\left(1-C \mu^{1 / 2}\right)\left\|q^{w} v\right\|^{2}+\mu\left(1-C \mu^{1 / 2}\right)\left\|\left\langle\mu D^{\prime}\right\rangle^{1 / 2} v\right\|^{2}
$$

because one can write $\mu S\left(r\left\langle\mu \xi^{\prime}\right\rangle, g\right)=C \# q+\mu^{3 / 2} S\left(\left\langle\mu \xi^{\prime}\right\rangle^{1 / 2}, g\right)$ with $C \in \mu S(1, g)$. Noting that

$$
\begin{aligned}
\left\langle\mu D^{\prime}\right\rangle^{\kappa^{\prime}} \operatorname{Re} Q= & \left(\left\langle\mu \xi^{\prime}\right\rangle^{\kappa^{\prime}} q^{2}\right)^{w}+\mu\left\langle\mu D^{\prime}\right\rangle^{1+\kappa^{\prime}} \\
& +\mu^{3 / 4} S\left(r\left\langle\mu \xi^{\prime}\right\rangle^{\kappa^{\prime}+5 / 4}, g\right)+\mu^{2} S\left(\left\langle\mu \xi^{\prime}\right\rangle^{\kappa^{\prime}}, g\right),
\end{aligned}
$$

one can write $S\left(r\left\langle\mu \xi^{\prime}\right\rangle^{\kappa^{\prime}+5 / 4}, g\right)=C \#\left(\left\langle\mu \xi^{\prime}\right\rangle^{\kappa^{\prime} / 2} q\right)+\mu S\left(\left\langle\mu \xi^{\prime}\right\rangle^{1 / 2+\kappa^{\prime}}, g\right)$ with $C \in$ $S\left(\left\langle\mu \xi^{\prime}\right\rangle^{1 / 4+\kappa^{\prime} / 2}, g\right)$, and we have

$$
\begin{aligned}
& \operatorname{Re}\left(\left\langle\mu D^{\prime}\right\rangle^{\kappa^{\prime}}(\operatorname{Re} Q) v, v\right) \geq\left(\left(\left\langle\mu \xi^{\prime}\right\rangle^{\kappa^{\prime}} q^{2}\right)^{w} v, v\right)+\mu\left\|\left\langle\mu D^{\prime}\right\rangle^{\kappa^{\prime} / 2+1 / 2} v\right\|^{2} \\
& \quad-C \mu^{1 / 4}\left\|\left(\left\langle\mu \xi^{\prime}\right\rangle^{\kappa^{\prime} / 2} q\right)^{w} v\right\|^{2}-C \mu^{5 / 4}\left\|\left\langle\mu D^{\prime}\right\rangle^{1 / 4+\kappa^{\prime} / 2} v\right\|^{2} .
\end{aligned}
$$

On the other hand, since

$$
\left\langle\mu \xi^{\prime}\right\rangle^{\kappa^{\prime}} q^{2}=\left(\left\langle\mu \xi^{\prime}\right\rangle^{\kappa^{\prime} / 2} q\right) \#\left(\left\langle\mu \xi^{\prime}\right\rangle^{\kappa^{\prime} / 2} q\right)+\mu S\left(r\left\langle\mu \xi^{\prime}\right\rangle^{1+\kappa^{\prime}}, g\right)+\mu^{3 / 2} S\left(\left\langle\mu \xi^{\prime}\right\rangle^{\kappa^{\prime}+1 / 2}, g\right)
$$

it follows that

$$
\left(\left(\left\langle\mu \xi^{\prime}\right\rangle^{\kappa^{\prime}} q^{2}\right)^{w} v, v\right) \geq\left(1-C \mu^{1 / 2}\right)\left\|\left(\left\langle\mu \xi^{\prime}\right\rangle^{\kappa^{\prime} / 2} q\right)^{w} v\right\|^{2}-C \mu^{3 / 2}\left\|\left\langle\mu D^{\prime}\right\rangle^{1 / 4+\kappa^{\prime} / 2} u\right\|^{2}
$$

and hence

$$
\begin{aligned}
\operatorname{Re}\left(\left\langle\mu D^{\prime}\right\rangle^{\kappa^{\prime}}(\operatorname{Re} Q) v, v\right) \geq & \left(1-C \mu^{1 / 4}\right)\left\|\left(\left\langle\mu \xi^{\prime}\right\rangle^{\kappa^{\prime} / 2} q\right)^{w} v\right\|^{2} \\
& +\mu\left(1-C \mu^{1 / 4}\right)\left\|\left\langle\mu D^{\prime}\right\rangle^{\kappa^{\prime} / 2+1 / 2} v\right\|^{2} .
\end{aligned}
$$

We estimate the terms $((\operatorname{Im} m) \Lambda v, \Lambda v)$ and $\operatorname{Re}((\operatorname{Re} Q) v,(\operatorname{Im} \lambda) v)$. Noting that $\operatorname{Im} m \in \mu S\left(\left\langle\mu \xi^{\prime}\right\rangle^{\kappa^{\prime}}, g\right)+\sqrt{\mu} S(1, g)$, it is clear that

$$
|((\operatorname{Im} m) \Lambda v, \Lambda v)| \leq C \mu\left\|\left\langle\mu D^{\prime}\right\rangle^{\kappa^{\prime} / 2} \Lambda v\right\|^{2}+C \sqrt{\mu}\|\Lambda v\|^{2} .
$$

With a large constant $K>0$, let us write

$$
\begin{aligned}
& \left(K\left(\mu\left\langle\mu \xi^{\prime}\right\rangle^{\kappa^{\prime}}+\sqrt{\mu}\right)-\operatorname{Im} \lambda\right) \#(\operatorname{Re} Q) \\
& =\left(\left(K\left(\mu\left\langle\mu \xi^{\prime}\right\rangle^{\kappa^{\prime}}+\sqrt{\mu}\right)-\operatorname{Im} \lambda\right)^{1 / 2} q\right) \#\left(\left(\left(K \mu\left\langle\mu \xi^{\prime}\right\rangle^{\kappa^{\prime}}+\sqrt{\mu}\right)-\operatorname{Im} \lambda\right)^{1 / 2} q\right) \\
& \quad+\mu^{5 / 4}\left(\left\langle\mu \xi^{\prime}\right\rangle^{\kappa^{\prime} / 2} q\right) \# C+\mu^{2} S\left(\left\langle\mu \xi^{\prime}\right\rangle^{\kappa^{\prime}+1 / 2}, g\right)
\end{aligned}
$$

with $C \in S\left(\left\langle\mu \xi^{\prime}\right\rangle^{1 / 4+\kappa^{\prime} / 2}, g\right)$ which shows that

$$
\begin{gathered}
K \mu \operatorname{Re}\left(\left\langle\mu D^{\prime}\right\rangle^{\kappa^{\prime}}(\operatorname{Re} Q) v, v\right)+K \sqrt{\mu}((\operatorname{Re} Q) v, v) \geq \operatorname{Re}((\operatorname{Re} Q) v,(\operatorname{Im} \lambda) v) \\
-C \mu\left\|\left(\left\langle\mu \xi^{\prime}\right\rangle^{\kappa^{\prime} / 2} q\right)^{w} v\right\|^{2}-C \mu^{3 / 2}\left\|\left\langle\mu D^{\prime}\right\rangle^{1 / 2+\kappa^{\prime} / 2} v\right\|^{2}
\end{gathered}
$$


and hence

$$
\operatorname{Re}((\operatorname{Re} Q) v,(\operatorname{Im} \lambda) v) \leq K^{\prime} \mu \operatorname{Re}\left(\left\langle\mu D^{\prime}\right\rangle^{\kappa^{\prime}}(\operatorname{Re} Q) v, v\right)+K^{\prime} \sqrt{\mu}((\operatorname{Re} Q) v, v)
$$

with some $K^{\prime}>0$.

Let us consider $\operatorname{Re}(\Lambda v,(\operatorname{Im} Q) v)$. Let $\operatorname{Im} Q=Q_{1} \in \mu S\left(w^{1 / 2}\left\langle\mu \xi^{\prime}\right\rangle^{1+\kappa}, g\right)$. Writing

$$
Q_{1}=\left(\mu r^{-1} w^{1 / 2}\left\langle\mu \xi^{\prime}\right\rangle^{\kappa / 2} a\right) \# A+\mu^{3 / 2} S\left(\left\langle\mu \xi^{\prime}\right\rangle^{\kappa+1 / 2}, g\right)
$$

with $A \in S\left(r\left\langle\mu \xi^{\prime}\right\rangle^{1+\kappa / 2}, g\right)$, we get

$$
\begin{aligned}
\left|\left(\Lambda v, Q_{1} v\right)\right| \leq & \mu^{5 / 4}\left\|\left(w^{1 / 2} r^{-1} a\left\langle\mu \xi^{\prime}\right\rangle^{\kappa / 2}\right)^{w} \Lambda v\right\|^{2} \\
& +\mu^{3 / 4}\|A v\|^{2}+C \mu^{3 / 2}\left\|\left\langle\mu D^{\prime}\right\rangle^{\kappa / 2+1 / 4} v\right\|^{2} .
\end{aligned}
$$

Writing $A=C \#\left(\left\langle\mu \xi^{\prime}\right\rangle^{\kappa^{\prime} / 2} q\right)+\mu^{1 / 2} S\left(\left\langle\mu \xi^{\prime}\right\rangle^{1 / 2+\kappa / 2}, g\right)$ with $C \in S(1, g)$, we have

$$
\mu^{3 / 4}\|A v\|^{2} \leq C \mu^{3 / 4}\left\|\left(\left\langle\mu \xi^{\prime}\right\rangle^{\kappa^{\prime} / 2} q\right)^{w} v\right\|^{2}+C \mu^{7 / 4}\left\|\left\langle\mu D^{\prime}\right\rangle^{1 / 2+\kappa^{\prime} / 2} v\right\|^{2}
$$

and hence by (6.1), (6.3),

$$
\begin{aligned}
|(\Lambda v,(\operatorname{Im} Q) v)| \leq & C \mu^{3 / 4} \operatorname{Re}\left(\left\langle\mu D^{\prime}\right\rangle^{\kappa^{\prime}}(\operatorname{Re} Q) v, v\right) \\
& +C \mu^{1 / 4}((\operatorname{Im} B) \Lambda v, \Lambda v)+C \mu^{5 / 4}\left\|\left\langle\mu D^{\prime}\right\rangle^{\kappa^{\prime} / 2} \Lambda v\right\|^{2}
\end{aligned}
$$

We turn to $\left(\left[D_{0}-\operatorname{Re} \lambda, \operatorname{Re} Q\right] v, v\right)$. Note that

$$
\begin{aligned}
{\left[D_{0}-\operatorname{Re} \lambda, \operatorname{Re} Q\right]=} & \frac{1}{i}\left(\left\{\xi_{0}+\lambda_{1}-k\left\langle\mu \xi^{\prime}\right\rangle^{-2} \phi_{1}^{3}+\operatorname{Re} \tilde{\lambda}, \phi_{2}^{2}+\alpha \theta^{2}\right\}\right)^{w} \\
& +\mu^{2} S\left(\left\langle\mu \xi^{\prime}\right\rangle^{\kappa^{\prime}+1}, g\right) .
\end{aligned}
$$

From (5.12) it follows that

$$
\left\{\xi_{0}+\lambda_{1}-k\left\langle\mu \xi^{\prime}\right\rangle^{-2} \phi_{1}^{3}, \phi_{2}^{2}\right\} \in \mu S\left(r^{2}\left\langle\mu \xi^{\prime}\right\rangle^{2}, g\right) .
$$

Recalling that $\theta=\beta \phi_{1}^{2}$ with some $\beta \in S\left(\left\langle\mu \xi^{\prime}\right\rangle^{-1}, g_{0}\right)$, it is clear that

$$
\left\{\xi_{0}+\lambda_{1}-k\left\langle\mu \xi^{\prime}\right\rangle^{-2} \phi_{1}^{3}, \alpha \theta^{2}\right\} \in \mu S\left(w^{3 / 2}\left\langle\mu \xi^{\prime}\right\rangle^{2}, g\right) \subset \mu S\left(r^{3 / 2}\left\langle\mu \xi^{\prime}\right\rangle^{2}, g\right) .
$$

Let $A \in \mu S\left(r^{3 / 2}\left\langle\mu \xi^{\prime}\right\rangle^{2}, g\right)$; then one can write

$$
A=\mu^{1 / 4}\left(\left\langle\mu \xi^{\prime}\right\rangle^{\kappa^{\prime} / 2} q\right) \# A_{1}+\mu^{7 / 4} S\left(\left\langle\mu \xi^{\prime}\right\rangle^{1+\kappa^{\prime}}, g\right)
$$

with $A_{1} \in \mu^{3 / 4} S\left(r^{1 / 2}\left\langle\mu \xi^{\prime}\right\rangle^{1-\kappa^{\prime} / 2}, g\right)$, and again we can write

$$
A_{1} \# A_{1}=\mu^{1 / 2}\left(\left\langle\mu \xi^{\prime}\right\rangle^{\kappa^{\prime} / 2} q\right) \# A_{2}+\mu^{2} S\left(\left\langle\mu \xi^{\prime}\right\rangle^{1+\kappa^{\prime}}, g\right)
$$

with $A_{2} \in \mu S\left(\left\langle\mu \xi^{\prime}\right\rangle^{1-3 \kappa^{\prime} / 2}, g\right) \subset \mu S\left(\left\langle\mu \xi^{\prime}\right\rangle^{1 / 2+\kappa^{\prime} / 2}, g\right)$. These prove that

$$
|(A v, v)| \leq C \mu^{1 / 2}\left\|\left(\left\langle\mu \xi^{\prime}\right\rangle^{\kappa^{\prime} / 2} q\right)^{w} v\right\|^{2}+C \mu^{7 / 4}\left\|\left\langle\mu D^{\prime}\right\rangle^{1 / 2+\kappa^{\prime} / 2} v\right\|^{2} .
$$

On the other hand it is easy to see

$$
\left\{\operatorname{Re} \tilde{\lambda}, \phi_{2}^{2}+\alpha \theta^{2}\right\}=\mu^{1 / 4}\left(\left\langle\mu \xi^{\prime}\right\rangle^{\kappa^{\prime} / 2} q\right) \# A_{3}+\mu^{2} S\left(\left\langle\mu \xi^{\prime}\right\rangle^{1+\kappa^{\prime}}, g\right)
$$

with $A_{3} \in \mu S\left(\left\langle\mu \xi^{\prime}\right\rangle^{1 / 2+\kappa^{\prime} / 2}, g\right)$. Hence taking (6.3) and (6.7) into account, we have

$$
C \sqrt{\mu} \operatorname{Re}\left(\left\langle\mu D^{\prime}\right\rangle^{\kappa^{\prime}}(\operatorname{Re} Q) v, v\right)+\operatorname{Im}\left(\left[D_{0}-\operatorname{Re} \lambda, \operatorname{Re} Q\right] v, v\right) \geq 0
$$

with some $C>0$. 
Finally, we consider $P_{1}=S(1, \bar{g}) \Lambda+\mu S\left(r\left\langle\mu \xi^{\prime}\right\rangle^{1+\kappa^{\prime}}, g\right)+\mu S\left(\left\langle\mu \xi^{\prime}\right\rangle, \bar{g}\right)$. We first study the term $A \in \mu S\left(\left\langle\mu \xi^{\prime}\right\rangle, \bar{g}\right)$ and estimate $|(A v, \Lambda v)|$. Write

$$
A=\mu^{5 / 8}\left(w^{1 / 2} r^{-1} a\left\langle\mu \xi^{\prime}\right\rangle^{\kappa / 2}\right) \#\left(\mu^{-5 / 8} A^{\prime}\right)+\mu^{5 / 4} S\left(\left\langle\mu \xi^{\prime}\right\rangle^{3 / 4}, \bar{g}\right)
$$

with $A^{\prime} \in \mu S\left(w^{-1 / 2} r\left\langle\mu \xi^{\prime}\right\rangle^{1-\kappa / 2}, \bar{g}\right)$. Thus we have

$$
\begin{aligned}
|(A v, \Lambda v)| \leq & C \mu^{5 / 4}\left\|\left(w^{1 / 2} r^{-1} a\left\langle\mu \xi^{\prime}\right\rangle^{\kappa / 2}\right)^{w} \Lambda v\right\|^{2}+C \mu^{-5 / 4}\left\|\left(A^{\prime}\right)^{w} v\right\|^{2} \\
& +C \mu\left\|\left\langle\mu D^{\prime}\right\rangle^{\kappa^{\prime} / 2} \Lambda v\right\|^{2}+C \mu^{3 / 2}\left\|\left\langle\mu D^{\prime}\right\rangle^{1 / 2+\kappa^{\prime} / 2} v\right\|^{2} .
\end{aligned}
$$

Since $r^{2} w^{-1}\left\langle\mu \xi^{\prime}\right\rangle^{2-\kappa}=r^{2}\left\langle\mu \xi^{\prime}\right\rangle^{2+\kappa^{\prime}} w^{-1}\left\langle\mu \xi^{\prime}\right\rangle^{-1 / 2} \leq \mu^{-1 / 2} r^{2}\left\langle\mu \xi^{\prime}\right\rangle^{2+\kappa^{\prime}}$ so that

$$
\mu^{-5 / 4} A^{\prime} \# A^{\prime} \in \mu^{1 / 4} S\left(r^{2}\left\langle\mu \xi^{\prime}\right\rangle^{2+\kappa^{\prime}}, \bar{g}\right) \text {, }
$$

one can write

$$
\begin{aligned}
K \mu^{1 / 4}\left\langle\mu \xi^{\prime}\right\rangle^{\kappa^{\prime}} q^{2}-\mu^{-5 / 4} A^{\prime} \# A^{\prime}= & \mu^{1 / 4}\left(b\left\langle\mu \xi^{\prime}\right\rangle^{\kappa^{\prime} / 2} q\right) \#\left(b\left\langle\mu \xi^{\prime}\right\rangle^{\kappa^{\prime} / 2} q\right) \\
& +\mu^{3 / 4}\left(\left\langle\mu \xi^{\prime}\right\rangle^{\kappa^{\prime} / 2} q\right) \# C+\mu^{5 / 4} S\left(\left\langle\mu \xi^{\prime}\right\rangle^{1+\kappa^{\prime}}, \bar{g}\right)
\end{aligned}
$$

with $b=\sqrt{K-\mu^{-3 / 2}\left\langle\mu \xi^{\prime}\right\rangle^{-\kappa^{\prime}} q^{-2}\left(A^{\prime} \# A^{\prime}\right)} \in S(1, g)$ with a large $K>0$ and $C \in$ $S\left(\left\langle\mu \xi^{\prime}\right\rangle^{1 / 2+\kappa^{\prime} / 2}, \bar{g}\right)$. This shows that

$$
\begin{aligned}
C \mu^{1 / 4}\left(\left(\left\langle\mu \xi^{\prime}\right\rangle^{\kappa^{\prime}} q^{2}\right)^{w} v, v\right) \geq & \mu^{-5 / 4}\left\|\left(A^{\prime}\right)^{w} v\right\|^{2} \\
& -C \mu^{1 / 4}\left\|\left(\left\langle\mu \xi^{\prime}\right\rangle^{\kappa^{\prime} / 2} q\right)^{w} v\right\|^{2}-C \mu^{5 / 4}\left\|\left\langle\mu D^{\prime}\right\rangle^{1 / 2+\kappa^{\prime} / 2} v\right\|^{2} .
\end{aligned}
$$

Thus we get, from (6.1) and (6.3),

$$
\begin{aligned}
|(A v, \Lambda v)| \leq & C \mu^{1 / 4}((\operatorname{Im} B) \Lambda v, \Lambda v) \\
& +C \mu^{1 / 4} \operatorname{Re}\left(\left\langle\mu D^{\prime}\right\rangle^{\kappa^{\prime}}(\operatorname{Re} Q) v, v\right)+C \mu\left\|\left\langle\mu D^{\prime}\right\rangle^{\kappa^{\prime} / 2} \Lambda v\right\|^{2} .
\end{aligned}
$$

In particular, this shows that one can control any lower-order term by the righthand side of (6.9). We next estimate $|(A v, \Lambda v)|, A \in \mu S\left(r\left\langle\mu \xi^{\prime}\right\rangle^{1+\kappa^{\prime}}, g\right)$. Write

$$
A=\mu\left\langle\mu \xi^{\prime}\right\rangle^{\kappa^{\prime} / 2} \# A^{\prime}+\mu^{3 / 2} S\left(\left\langle\mu \xi^{\prime}\right\rangle^{\kappa^{\prime}+1 / 2}, \bar{g}\right), \quad A^{\prime} \in S\left(r\left\langle\mu \xi^{\prime}\right\rangle^{1+\kappa^{\prime} / 2}, \bar{g}\right),
$$

and hence

$$
|(A v, \Lambda v)| \leq C \mu\left\|\left\langle\mu D^{\prime}\right\rangle^{\kappa^{\prime} / 2} \Lambda v\right\|^{2}+C \mu\left\|\left(A^{\prime}\right)^{w} v\right\|^{2}+C \mu^{2}\left\|\left\langle\mu D^{\prime}\right\rangle^{\kappa^{\prime} / 2+1 / 2} v\right\|^{2} .
$$

Writing $A^{\prime}=\left\langle\mu \xi^{\prime}\right\rangle^{\kappa^{\prime} / 2} q \# C+\mu^{1 / 2} S\left(\left\langle\mu \xi^{\prime}\right\rangle^{\kappa^{\prime} / 2+1 / 2}, \bar{g}\right)$ with $C \in S(1, \bar{g})$, we get

$$
|(A v, \Lambda v)| \leq C \mu\left\|\langle\mu D\rangle^{\kappa^{\prime} / 2} \Lambda v\right\|^{2}+C \mu \operatorname{Re}\left(\left\langle\mu D^{\prime}\right\rangle^{\kappa^{\prime}}(\operatorname{Re} Q) v, v\right) .
$$

For $A \in S(1, \bar{g})$ it is clear that

$$
|(A \Lambda v, \Lambda v)| \leq C\|\Lambda v\|^{2} .
$$

From (6.1) to $(6.11)$ we now have

$$
\begin{aligned}
\operatorname{Im}(\tilde{P} v, \Lambda v)= & \operatorname{Im}\left(\left(\tilde{P}-\tilde{P}_{1}\right) v, \Lambda v\right)+\operatorname{Im}\left(\tilde{P}_{1} v, \Lambda v\right) \\
& \geq \frac{d}{d x_{0}}\left(\|\Lambda v\|^{2}+((\operatorname{Re} Q) v, v)\right)+\left(1-C \mu^{3 / 4}\right)\left\|\left\langle\mu D^{\prime}\right\rangle^{\kappa^{\prime} / 2} \Lambda v\right\|^{2} \\
& +\mu\left(1-C \mu^{1 / 4}\right)\left\|\left\langle\mu D^{\prime}\right\rangle^{\kappa^{\prime} / 2+1 / 2} v\right\|^{2}-C \mu((\operatorname{Re} Q) v, v)-C\|\Lambda v\|^{2} .
\end{aligned}
$$


Multiplying (6.12) by $e^{-\gamma x_{0}}$ we get

$$
\begin{aligned}
& e^{-\gamma x_{0}}\left\|\left\langle\mu D^{\prime}\right\rangle^{-\kappa^{\prime} / 2} \tilde{P} v\right\|^{2} \\
& \geq \frac{d}{d x_{0}}\left[e^{-\gamma x_{0}}\left(\|\Lambda v\|^{2}+((\operatorname{Re} Q) v, v)\right)\right] \\
& \quad+\left(1-C \mu^{3 / 4}\right) e^{-\gamma x_{0}}\left\|\left\langle\mu D^{\prime}\right\rangle^{\kappa^{\prime} / 2} \Lambda v\right\|^{2} \\
& \quad+\mu\left(1-C \mu^{1 / 4}\right) e^{-\gamma x_{0}}\left\|\left\langle\mu D^{\prime}\right\rangle^{\kappa^{\prime} / 2+1 / 2} v\right\|^{2} \\
& \quad+(\gamma-C) e^{-\gamma x_{0}}\|\Lambda v\|^{2}+(\gamma-C \mu) e^{-\gamma x_{0}}((\operatorname{Re} Q) v, v) .
\end{aligned}
$$

Thus for $0<\mu<\mu_{0}$ and $\gamma>\gamma_{0}\left(\mu_{0}\right)$, taking (6.2) into account, one has

$$
\begin{aligned}
& \int_{-T}^{t} e^{-\gamma x_{0}}\left\|\left\langle\mu D^{\prime}\right\rangle^{-\kappa^{\prime} / 2} \tilde{P} v\right\|^{2} d x_{0} \\
& \geq c e^{-\gamma t}\left(\|\Lambda v(t)\|^{2}+\mu\left\|\left\langle\mu D^{\prime}\right\rangle^{1 / 2} v(t)\right\|^{2}\right) \\
& \quad+c \int_{-T}^{t} e^{-\gamma x_{0}}\left\{\left\|\left\langle\mu D^{\prime}\right\rangle^{\kappa^{\prime} / 2} \Lambda v\right\|^{2}+\mu\left\|\left\langle\mu D^{\prime}\right\rangle^{\kappa^{\prime} / 2+1 / 2} v\right\|^{2}\right\} d x_{0} \\
& \quad+c \gamma \int_{-T}^{t} e^{-\gamma x_{0}}\left\{\|\Lambda v\|^{2}+\mu\left\|\left\langle\mu D^{\prime}\right\rangle^{1 / 2} v\right\|^{2}\right\} d x_{0}
\end{aligned}
$$

for $v \in C^{2}\left((-T, T) ; \mathcal{S}\left(\mathbb{R}^{n}\right)\right)$ vanishing in $x_{0} \leq 0$.

Let $h\left(x^{\prime}, \xi^{\prime}\right) \in S_{(s)}\left(1, g_{0}\right)$, and assume $\left\{\Lambda, x_{0}+h\right\} \geq c>0$ with some $c>0$. Then it is easy to see that the a priori estimates (6.13) holds with phase function

$$
\tilde{\phi}_{h}=-\left(x_{0}+h\right)\left\langle\mu \xi^{\prime}\right\rangle^{\kappa^{\prime}}-2\left\langle\mu \xi^{\prime}\right\rangle^{\kappa} \arg \left(\phi_{2}+i w\right)
$$

since $\left\langle\mu \xi^{\prime}\right\rangle^{\kappa^{\prime}} h \in S_{(s)}\left(\left\langle\mu \xi^{\prime}\right\rangle^{\kappa^{\prime}}, g_{0}\right)$, and hence $\left\{\Lambda,\left\langle\mu \xi^{\prime}\right\rangle^{\kappa^{\prime}} h\right\} \in \mu S_{(s)}\left(\left\langle\mu \xi^{\prime}\right\rangle^{\kappa^{\prime}}, g_{0}\right)$. Starting with $P^{*}$ instead of $P$, we get $\operatorname{Op}^{0}\left(e^{\tilde{\phi}_{h}}\right) P^{*} \operatorname{Op}^{1}\left(e^{-\tilde{\phi}_{h}}\right) \operatorname{Op}^{0}\left(j_{h}\right)$, where $\mathrm{Op}^{0}\left(e^{\tilde{\phi}_{h}}\right) \mathrm{Op}^{1}\left(e^{-\tilde{\phi}_{h}}\right) \mathrm{Op}^{0}\left(j_{h}\right)=I$. Taking its adjoint we have

$$
T_{h} P S_{h}=\tilde{P}_{h},
$$

where

$$
T_{h}=\mathrm{Op}^{1}\left(\bar{j}_{h}\right) \mathrm{Op}^{0}\left(e^{-\tilde{\phi}_{h}}\right), \quad S_{h}=\mathrm{Op}^{1}\left(e^{\tilde{\phi}_{h}}\right)
$$

because $\mathrm{Op}^{0}(p)^{*}=\mathrm{Op}^{1}(\bar{p})$. Recall that $T_{h} S_{h}=S_{h} T_{h}=I$. We prove the existence of a parametrix of finite propagation speed of $P$ by using the a priori estimates for $\tilde{P}_{h}$. Recall that $P$ can be assumed to be of the form (2.2).

As for the fourth term in the right-hand side of (2.2), assuming that $s$ verifies $(1+3 \rho) s \kappa^{\prime}<1$, we apply the following $(\rho=3 / 4, \delta=1 / 2)$.

\section{LEMMA 6.1}

Let $s_{1}>s$ and $1>s_{1} \kappa^{\prime}$, and assume $R \in S_{\left(s_{1}\right)}\left(e^{-c_{1}\left\langle\mu \xi^{\prime}\right\rangle^{1 / s}}, g_{\rho}\right)$ with $g_{\rho}=|d x|^{2}+$ $\left\langle\xi^{\prime}\right\rangle_{\mu}^{-2 \rho}\left|d \xi^{\prime}\right|^{2}$. Then we have

$$
\mathrm{Op}^{0}\left(e^{\tilde{\phi}_{h}}\right) \mathrm{Op}^{0}(R) \mathrm{Op}^{1}\left(e^{-\tilde{\phi}_{h}}\right)=\mathrm{Op}^{0}(c),
$$


where

$$
c \in S_{\left((1+\rho) s_{1}+\rho s /(1-\delta)\right)}\left(e^{-c_{2}\left\langle\xi^{\prime}\right\rangle^{s_{2}}}, \bar{g}\right)
$$

with $s_{2}=\min \left\{1 / s_{1},(1-\delta) / s\right\}$. In particular, $c \in S\left(\left\langle\mu \xi^{\prime}\right\rangle^{\ell}, \bar{g}\right)$ for any $\ell \in \mathbb{Z}$.

Proof

We sketch the proof. To simplify notations we write $\tilde{\phi}$ for $\tilde{\phi}_{h}$. Let $\operatorname{Op}^{0}\left(e^{\tilde{\phi}}\right) \times$ $\mathrm{Op}^{0}(R)=\mathrm{Op}^{0}(b)$. Then repeating arguments similar to those in the proof of Proposition 2.1, we see that

$$
b \in S_{\left((1+\rho) s_{1}\right)}\left(e^{-c\left\langle\mu \xi^{\prime}\right\rangle^{1 / s_{1}}}, \bar{g}\right) .
$$

We next consider $\mathrm{Op}^{0}(b) \mathrm{Op}^{1}\left(e^{-\tilde{\phi}}\right)=\mathrm{Op}^{0}\left(c_{1}\right)+\mathrm{Op}^{0}\left(c_{2}\right)$, where

$$
c_{i}=(2 \pi)^{-n} \int e^{-i y^{\prime} \eta^{\prime}-\tilde{\phi}\left(x^{\prime}+y^{\prime}, \xi^{\prime}+\eta^{\prime}\right)} \chi_{i} b\left(x^{\prime}, \xi^{\prime}+\eta^{\prime}\right) d y^{\prime} d \eta^{\prime}
$$

with $\chi_{1}=\chi\left(\left(\eta^{\prime}-\xi^{\prime}\right)\left\langle\xi^{\prime}\right\rangle_{\mu}^{-1}\right), \chi_{2}=1-\chi_{1}$. It is easy to check that

$$
c_{1} \in S_{(\hat{s})}\left(e^{-c\left\langle\mu \xi^{\prime}\right\rangle^{1 / s_{1}}}, \bar{g}\right)
$$

with $\hat{s}=(1+\rho) s_{1}$. We consider $c_{2}$. Note that

$$
\begin{aligned}
& \left|\partial_{x^{\prime}}^{\beta} \partial_{\xi^{\prime}}^{\alpha}\left\langle D_{y^{\prime}}\right\rangle^{N}\left\langle\eta^{\prime}\right\rangle^{-N} e^{-\tilde{\phi}\left(x^{\prime}+y^{\prime}, \xi^{\prime}+\eta^{\prime}\right)} \chi_{2} b\left(x^{\prime}, \xi^{\prime}+\eta^{\prime}\right)\right| \\
& \quad \leq C A^{|\alpha+\beta|}|\alpha+\beta| !^{\hat{s}}\left\langle\eta^{\prime}\right\rangle^{\delta|\beta|+\rho|\alpha|}\left\langle\xi^{\prime}\right\rangle_{\mu}^{-\rho|\alpha|} e^{c\left\langle\mu \eta^{\prime}\right\rangle^{\kappa^{\prime}}} A^{N} N !^{s}\left\langle\eta^{\prime}\right\rangle^{-(1-\delta) N} .
\end{aligned}
$$

Choose $N$ so that $N=[(\rho|\alpha|+\delta|\beta|+\ell) /(1-\delta)], \ell \in \mathbb{N}$, and hence the right-hand side is bounded by

$$
C A^{|\alpha+\beta|}|\alpha+\beta| !^{\hat{s}+\rho s /(1-\delta)}\left\langle\xi^{\prime}\right\rangle_{\mu}^{-\rho|\alpha|} e^{c\left\langle\mu \eta^{\prime}\right\rangle^{\kappa^{\prime}}}\left(\frac{A \ell^{s /(1-\delta)}}{\left\langle\eta^{\prime}\right\rangle}\right)^{\ell} .
$$

Taking $\ell$ such that $\ell=\left[\left(A^{-1} e^{-1}\left\langle\eta^{\prime}\right\rangle\right)^{(1-\delta) / s}\right]$ and noting $(1-\delta) / s>\kappa^{\prime}$, we conclude that

$$
c_{2} \in S_{(\hat{s}+\rho s /(1-\delta))}\left(e^{-c\left\langle\xi^{\prime}\right\rangle_{\mu}^{(1-\delta) / s}}, g_{\rho}\right) .
$$

These prove the desired assertion.

Let us fix a small $T>0$ and $h=\epsilon$. Since the energy estimates (6.13) hold for $\tilde{P}_{\epsilon}$, then from the standard arguments on functional analysis we conclude that for any given $F \in C^{0}\left([-T, T] ; H^{\infty}\left(\mathbb{R}^{n}\right)\right)$ vanishing in $x_{0} \leq 0$, there is a unique $U \in C^{2}\left([-T, T] ; H^{\infty}\left(\mathbb{R}^{n}\right)\right)$ vanishing in $x_{0}<0$ such that $\tilde{P}_{\epsilon} U=F$. Let $1 \leq \bar{s}<4$, and let $f \in C^{0}\left([-T, T] ; \gamma_{0}^{(\bar{s})}\left(\mathbb{R}^{n}\right)\right)$ be such that $f(x)=0$ for $x_{0} \leq 0$. We choose $\kappa^{\prime}$ and $\kappa$ such that

$$
\bar{s}<\frac{1}{\kappa^{\prime}}<4, \quad \kappa^{\prime}+\kappa=\frac{1}{2} .
$$

Since $-\tilde{\phi}_{\epsilon} \leq C\left\langle\mu \xi^{\prime}\right\rangle^{\kappa^{\prime}}$, it is easy to see that $\mathrm{Op}^{0}\left(e^{-\tilde{\phi}_{\epsilon}}\right) f \in C^{0}\left([-T, T] ; H^{\infty}\left(\mathbb{R}^{n}\right)\right)$ because $1 / \bar{s}>\kappa^{\prime}$, and hence $F=T_{\epsilon} f \in C^{0}\left([-T, T] ; H^{\infty}\left(\mathbb{R}^{n}\right)\right)$. Then as remarked 
above, there exists a unique $U \in C^{2}\left([-T, T] ; H^{\infty}\left(\mathbb{R}^{n}\right)\right)$ such that

$$
T_{\epsilon} P S_{\epsilon} U=\tilde{P}_{\epsilon} U=F=T_{\epsilon} f,
$$

where $U=0$ in $x_{0} \leq 0$. This implies that $P\left(S_{\epsilon} U\right)=f$. Let us denote

$$
u=S_{\epsilon} U=G f
$$

and prove that $G$ is a parametrix of $P$ with finite propagation speed.

We first examine $u=S_{\epsilon} U \in C^{2}\left([-T, T] ; H^{\infty}\left(\mathbb{R}^{n}\right)\right)$. Since $\tilde{\phi}_{\epsilon} \leq-\epsilon\left\langle\mu \xi^{\prime}\right\rangle^{\kappa^{\prime}}$ for

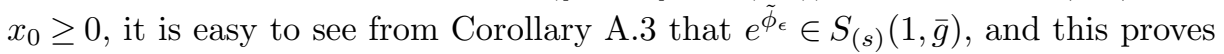
the assertion. We now prove that $G$ is a parametrix with finite propagation speed. Let $h_{1}\left(x^{\prime}, \xi^{\prime}\right) \in S_{(s)}\left(1, g_{0}\right)$ be such that

$$
\operatorname{supp} h_{1} \cap\left\{0 \leq x_{0} \leq \tau\right\} \subset\left\{x_{0}+h<0\right\} .
$$

\section{LEMMA 6.2}

Assume (6.15) and $\rho>s \kappa^{\prime}$. Then we have

$$
\mathrm{Op}^{0}\left(e^{-\tilde{\phi}_{h}}\right) \operatorname{Op}^{0}\left(h_{1}\right)=\mathrm{Op}^{0}\left(S_{((1+\rho) s)}\left(\left\langle\mu \xi^{\prime}\right\rangle^{\ell}, \bar{g}\right)\right), \quad 0 \leq x_{0} \leq \tau,
$$

for any $\ell \in \mathbb{Z}$.

\section{Proof}

We sketch the proof. Write $\mathrm{Op}^{0}\left(e^{-\tilde{\phi}_{h}}\right) \mathrm{Op}^{0}\left(h_{1}\right)=\mathrm{Op}^{0}\left(b_{1}\right)+\mathrm{Op}^{0}\left(b_{2}\right)$, where

$$
\mathrm{Op}^{0}\left(b_{i}\right) u=(2 \pi)^{-n} \int e^{-i y^{\prime} \eta^{\prime}+\tilde{\phi}_{h}\left(x, \xi^{\prime}+\eta^{\prime}\right)} \chi_{i}\left(\xi^{\prime}, \eta^{\prime}\right) h_{1}\left(x^{\prime}+y^{\prime}, \xi^{\prime}\right) u\left(y^{\prime}\right) d y^{\prime} d \eta^{\prime}
$$

with $\chi_{1}=\chi\left(M \eta^{\prime}\left\langle\xi^{\prime}\right\rangle_{\mu}^{-1}\right), \chi_{2}=1-\chi_{1}$. We consider only $\mathrm{Op}^{0}\left(b_{1}\right)$. Let us write $\mathrm{Op}^{0}\left(b_{1}\right)=\mathrm{Op}^{0}\left(b_{11}\right)+\mathrm{Op}^{0}\left(b_{12}\right)$, where

$\mathrm{Op}^{0}\left(b_{1 i}\right) u=(2 \pi)^{-n} \int e^{-i y^{\prime} \eta^{\prime}+\tilde{\phi}_{h}\left(x, \xi^{\prime}+\eta^{\prime}\right)} \tilde{\chi}_{i}\left(y^{\prime}\right) \chi_{1}\left(\xi^{\prime}, \eta^{\prime}\right) h_{1}\left(x^{\prime}+y^{\prime}, \xi^{\prime}\right) u\left(y^{\prime}\right) d y^{\prime} d \eta^{\prime}$

with $\tilde{\chi}_{1}\left(y^{\prime}\right)=\chi\left(K y^{\prime}\right)$ and $\tilde{\chi}_{2}=1-\tilde{\chi}_{1}$. Choose $K>0$ large so that

$$
-\tilde{\phi}_{h}\left(x, \xi^{\prime}+\eta^{\prime}\right) \leq-c^{\prime}\left\langle\mu \xi^{\prime}\right\rangle^{\kappa^{\prime}}, \quad 0 \leq x_{0} \leq \tau,
$$

on the support of $\chi_{1}\left(\xi^{\prime}, \eta^{\prime}\right) \tilde{\chi}_{1}\left(y^{\prime}\right)$ with some $c^{\prime}>0$. Then from integration by parts we see $b_{11} \in S_{(s)}\left(e^{-c\left\langle\mu \xi^{\prime}\right\rangle^{\kappa^{\prime}}}, \bar{g}\right)$. We turn to $\mathrm{Op}^{0}\left(b_{12}\right)$. By Corollary A.3 we see

$$
\begin{aligned}
& \left|\partial_{x^{\prime}}^{\beta} \partial_{\xi^{\prime}}^{\alpha} \partial_{\eta^{\prime}}^{\gamma}\left(y^{\prime}\right)^{-\gamma} e^{\tilde{\phi}_{h}\left(x, \xi^{\prime}+\eta^{\prime}\right)} \chi_{1}\left(\xi^{\prime}, \eta^{\prime}\right) \tilde{\chi}_{2}\left(y^{\prime}\right) h_{1}\left(x^{\prime}+y^{\prime}, \xi^{\prime}\right)\right| \\
& \quad \leq C A^{|\alpha+\beta+\gamma|}|\alpha+\beta| !^{s}\left\langle\xi^{\prime}\right\rangle_{\mu}^{-\rho|\alpha|+\delta|\beta|}|\gamma| !^{!}\left\langle\xi^{\prime}\right\rangle_{\mu}^{-\rho|\gamma|} e^{c\left\langle\mu \xi^{\prime}\right\rangle^{\kappa^{\prime}}} .
\end{aligned}
$$

Take $\gamma$ such that $|\gamma|=\left[\left(A^{-1} e^{-1}\left\langle\xi^{\prime}\right\rangle_{\mu}^{\rho}\right)^{1 / s}\right]$ and hence the right-hand side is bounded by

$$
C A^{|\alpha+\beta|}|\alpha+\beta| !^{s}\left\langle\xi^{\prime}\right\rangle_{\mu}^{-\rho|\alpha|+\delta|\beta|} e^{-c^{\prime}\left\langle\mu \xi^{\prime}\right\rangle^{\rho / s}}
$$

because $\rho / s>\kappa^{\prime}$. This proves $b_{12} \in S_{(s)}\left(e^{-c^{\prime}\left\langle\mu \xi^{\prime}\right\rangle^{\rho / s}}, \bar{g}\right)$. It is not difficult to see that $b_{2} \in S_{((1+\rho) s)}\left(e^{-c^{\prime}\left\langle\xi^{\prime}\right\rangle_{\mu}^{1 / s}}, \bar{g}\right)$. These prove the assertion. 
We now consider $P u=h_{1} f$. From $T_{h} P S_{h} T_{h} u=T_{h} h_{1} f$ we have

$$
\tilde{P}_{h}\left(T_{h} u\right)=T_{h} h_{1} f .
$$

From Lemma 6.2 it follows that for any $p, q$ we have

$$
\left\|T_{h} h_{1} f\right\|_{p} \leq C_{p, q}\|f\|_{q}
$$

where $\|u\|_{p}=\|u\|_{H^{p}\left(\mathbb{R}^{n}\right)}$. Thus one has, from (6.13),

$$
\left\|T_{h} u\right\|_{p} \leq C_{p} \int^{t}\left\|T_{h} h_{1} f\right\|_{p} d x_{0} \leq C_{p, q} \int^{t}\|f\|_{q} d x_{0} .
$$

Assume that $h_{2} \in S_{(s)}\left(1, g_{0}\right)$ verifies

$$
\operatorname{supp} h_{2} \cap\left\{0 \leq x_{0} \leq \tau\right\} \subset\left\{x_{0}+h>0\right\} .
$$

A repetition of similar arguments proving Lemma 6.2 gives

$$
h_{2} S_{h} \in S_{((1+\rho) s)}\left(\left\langle\mu \xi^{\prime}\right\rangle^{\ell}, \bar{g}\right)
$$

for $0 \leq x_{0} \leq \tau$ and for any $\ell \in \mathbb{Z}$, and hence

$$
\left\|h_{2} G h_{1} f\right\|_{p}=\left\|h_{2} u\right\|_{p}=\left\|h_{2} S_{h} T_{h} u\right\|_{p} \leq C_{p, q}\left\|T_{h} u\right\|_{p} \leq C_{p, q} \int^{t}\|f\|_{q} d x_{0}
$$

for any $p, q$.

Assume $\operatorname{supp} h_{1} \cap \operatorname{supp} h_{2}=\emptyset$. Then it is clear that one can take $\tau>0$ and $h$ satisfying (6.15) and (6.16). Indeed it is enough to take $h$ as a finite sum of such

$$
\epsilon \sqrt{\left|\xi^{\prime}\left\langle\xi^{\prime}\right\rangle_{\mu}^{-1}-\tilde{\xi}^{\prime}\left\langle\tilde{\xi}^{\prime}\right\rangle_{\mu}^{-1}\right|^{2}+\left|x^{\prime}-\tilde{x}^{\prime}\right|+\epsilon_{1}^{2}}-\epsilon_{2},
$$

where $0<\epsilon_{1}<\epsilon_{2}, 0<\epsilon<1$ are small. Thus we have proved that $G$ is a parametrix of $P$ with finite propagation speed at $\left(0, \hat{x}^{\prime}, \hat{\xi}^{\prime}\right)$.

Since the existence of a parametrix with finite propagation speed is invariant under conjugation of Fourier integral operators, then the original operator (1.1) has a parametrix with finite propagation speed at every $\left(0, x^{\prime}, \xi^{\prime}\right),\left|\xi^{\prime}\right| \neq 0$. Then it follows (see the proof of Proposition A.6 in [11]) that there is $\tau_{1}>0$ such that for any $f \in C^{0}\left([-T, T] ; \gamma_{0}^{(\bar{s})}\left(\mathbb{R}^{n}\right)\right)$ vanishing in $x_{0} \leq 0$ there exists a unique $u \in C^{2}\left(\left[-\tau_{1}, \tau_{1}\right] ; H^{\infty}\left(\mathbb{R}^{n}\right)\right)$ vanishing in $x_{0} \leq 0$ verifying (1.8).

\section{Appendices}

\section{A}

Here and in Appendix B we collect several results about symbol classes used in this paper and Fourier integral operators with complex phase function (4.4) without proofs. We refer to [17] for the proofs.

\section{A.1 Almost analytic extension of Gevrey functions}

In this subsection we study an almost analytic extension of $a(x, \xi, \mu) \in S_{(s)}(m, g)$ with

$$
g_{x, \xi}(y, \eta)=\sum_{j=1}^{n} \delta_{j}^{2} y_{j}^{2}+\rho_{j}^{-2} \eta_{j}^{2}
$$


where $\delta=\left(\delta_{1}(x, \xi, \mu), \ldots, \delta_{n}(x, \xi, \mu)\right), \rho=\left(\rho_{1}(x, \xi, \mu), \ldots, \rho_{n}(x, \xi, \mu)\right)$ and $\delta_{j}(x$, $\xi, \mu), \rho_{j}(x, \xi, \mu)$ are assumed to be in $S_{(s)}\left(\delta_{j}, g\right), S_{(s)}\left(\rho_{j}, g\right)$, respectively. Let $\chi(t) \in \gamma_{0}^{(s)}(\mathbb{R})$ which is 1 in $|t| \leq 1 / 4$ and 0 in $|t| \geq 1 / 2$, and set

$$
\chi(x)=\chi\left(x_{1}\right) \chi\left(x_{2}\right) \cdots \chi\left(x_{n}\right) .
$$

In what follows, to simplify notation we often drop a small parameter $\mu>0$.

We assume that there exists a metric

$$
g \leq \hat{g}_{\xi}(y, \eta)=\sum_{j=1}^{n}\langle\xi\rangle_{\mu}^{2 a_{j}} y_{j}^{2}+\langle\xi\rangle_{\mu}^{-2 b_{j}} \eta_{j}^{2}
$$

where $0 \leq a_{j}<b_{j} \leq 1$. Let us set

$$
\hat{g}_{\xi}^{\sigma}(y, \eta)=\sum_{j=1}^{n}\langle\xi\rangle_{\mu}^{2 b_{j}} y_{j}^{2}+\langle\xi\rangle_{\mu}^{-2 a_{j}} \eta_{j}^{2}, \quad h(\xi, \mu)=\langle\xi\rangle_{\mu}^{-\min _{i, j}\left(b_{i}-a_{j}\right)} .
$$

Let $k(\xi, \mu) \in S_{(s)}(k, \hat{g})$, and set

$$
\begin{aligned}
E(k) & =\left\{(x, y, \xi, \eta) \in \mathbb{R}^{4 n} \mid \hat{g}_{\xi}^{\sigma}(y, \eta)<k(\xi, \mu)^{2}\right\}, \\
E^{0}(k) & =\left\{(x, \xi, \eta) \in \mathbb{R}^{3 n} \mid \hat{g}_{\xi}^{\sigma}(0, \eta)<k(\xi, \mu)^{2}\right\} .
\end{aligned}
$$

We denote by $S_{(s)}(m, g \mid E(k))$ the set of all $a(x, y, \xi, \eta) \in C^{\infty}(E(k))$ verifying

$$
\left|\partial_{x, y}^{\beta} \partial_{\xi, \eta}^{\alpha} a(x, y, \xi, \eta)\right| \leq C A^{|\alpha+\beta|}|\alpha+\beta| !^{s} m \delta^{\beta} \rho^{-\alpha}, \quad \forall \alpha, \beta,
$$

for $(x, y, \xi, \eta) \in E(k)$ with some $C>0, A>0$. Similarly we define $S_{(s)}(m, \hat{g} \mid$ $\left.E^{0}(k)\right)$. Let

$$
d_{j}=B j^{s-1}, \quad j \geq 1, d_{0}=1,
$$

where $B$ is some positive constant, take $k(\xi, \mu) \in S_{(s)}(k, \hat{g})$ such that

$$
k(\xi, \mu) h(\xi, \mu) \leq C\langle\xi\rangle_{\mu}^{-\epsilon}
$$

with some $\epsilon>0$, and define an almost analytic extension $\tilde{a}(z, \zeta)=a(x+i y, \xi+i \eta)$ of $a(x, \xi) \in S_{(s)}(m, g)$ by

$$
a(x+i y, \xi+i \eta)=\sum_{\alpha, \beta} \frac{1}{\alpha ! \beta !} \partial_{x}^{\beta} \partial_{\xi}^{\alpha} a(x, \xi)(i y)^{\beta}(i \eta)^{\alpha} \chi\left(k d_{\beta}\langle\xi\rangle_{\mu}^{a-b}\right) \chi\left(k d_{\alpha}\langle\xi\rangle_{\mu}^{a-b}\right),
$$

where $d_{\beta}\langle\xi\rangle_{\mu}^{a-b}=\left(d_{\beta_{1}}\langle\xi\rangle_{\mu}^{a_{1}-b_{1}}, \ldots, d_{\beta_{n}}\langle\xi\rangle_{\mu}^{a_{n}-b_{n}}\right)$.

\section{PROPOSITION A.1}

Let $a(x, \xi) \in S_{(s)}(m, g)$. Then we have

$$
\tilde{a}(z, \zeta) \in S_{(s)}(m, g \mid E(k)) .
$$

Moreover, with $\bar{\partial}_{z_{j}}=\partial_{x_{j}}+i \partial_{y_{j}}$ and $\bar{\partial}_{\zeta_{j}}=\partial_{\xi_{j}}+i \partial_{\eta_{j}}$ we have, with some $c>0$,

$$
\begin{aligned}
& \bar{\partial}_{z_{j}} \tilde{a}(z, \zeta) \in S_{(s)}\left(m \delta^{e_{j}} e^{-c(h k)^{-1 /(s-1)}}, g \mid E(k)\right), \\
& \bar{\partial}_{\zeta_{j}} \tilde{a}(z, \zeta) \in S_{(s)}\left(m \rho^{-e_{j}} e^{-c(h k)^{-1 /(s-1)}}, g \mid E(k)\right) .
\end{aligned}
$$




\section{COROLLARY A.1}

Let $Y_{j} \in S_{(s)}\left(k\langle\xi\rangle_{\mu}^{-b_{j}}, g \mid E(k)\right), H_{j} \in S_{(s)}\left(k\langle\xi\rangle_{\mu}^{a_{j}}, g \mid E(k)\right)$, and let $Y=\left(Y_{1}, \ldots\right.$, $\left.Y_{n}\right), H=\left(H_{1}, \ldots, H_{n}\right)$. Then we have

$$
\tilde{a}(x, Y, \xi, H)-\sum_{|\alpha+\beta|<N} \frac{1}{\alpha ! \beta !} \partial_{x}^{\beta} \partial_{\xi}^{\alpha} a(x, \xi)(i Y)^{\beta}(i H)^{\alpha} \in S_{(s)}\left(m(k h)^{N}, g \mid E(k)\right)
$$

for any $N \in \mathbb{N}$.

\section{PROPOSITION A.2}

Let $\theta(x, \xi, v)$ be a positive function. Assume that $a(x, \xi, v) \in C^{\infty}\left(\mathbb{R}^{2 n} \times \Omega\right)$ verifies

$$
\left|\partial_{x}^{\beta} \partial_{\xi}^{\alpha} \partial_{v}^{\gamma} a(x, \xi, v)\right| \leq C A^{|\alpha+\beta+\gamma|}|\alpha+\beta+\gamma| !^{s} m \delta^{\beta} \rho^{-\alpha} \theta^{\gamma}, \quad(x, \xi, v) \in \mathbb{R}^{2 n} \times \Omega
$$

for all $\alpha, \beta, \gamma$. Then an almost analytic extension $\tilde{a}(z, \zeta, v)$ verifies

$$
\begin{aligned}
\left|\partial_{x, y}^{\beta} \partial_{\xi, \eta}^{\alpha} \partial_{v}^{\gamma} \tilde{a}(z, \zeta, v)\right| \leq & C A^{|\alpha+\beta+\gamma|}|\alpha+\beta+\gamma| !^{s} m \delta^{\beta} \rho^{-\alpha} \theta^{\gamma}, \\
\left|\partial_{x, y}^{\beta} \partial_{\xi, \eta}^{\alpha} \partial_{v}^{\gamma} \bar{\partial}_{z_{j}} \tilde{a}(z, \zeta, v)\right| \leq & C A^{|\alpha+\beta+\gamma|}|\alpha+\beta+\gamma| !^{s} \\
& \times m \delta^{e_{j}} e^{-c(h k)^{-1 /(s-1)} \delta^{\beta} \rho^{-\alpha} \theta^{\gamma}} \\
\left|\partial_{x, y}^{\beta} \partial_{\xi, \eta}^{\alpha} \partial_{v}^{\gamma} \bar{\partial}_{\zeta_{j}} \tilde{a}(z, \zeta, v)\right| \leq & C A^{|\alpha+\beta+\gamma|}|\alpha+\beta+\gamma| !^{s} \\
& \times m \rho^{-e_{j}} e^{-c(h k)^{-1 /(s-1)} \delta^{\beta} \rho^{-\alpha} \theta^{\gamma}}
\end{aligned}
$$

for $(x, y, \xi, \eta, v) \in E(k) \times \Omega$ and for any $\alpha, \beta, \gamma$.

\section{A.2 Estimates of composite functions}

Let us put

$$
\Gamma_{s}(k)=M \frac{k !^{s}}{k^{s+2}}
$$

Then we have the following.

\section{LEMMA A.1}

Choosing a positive constant $M$ suitably, we have

(i)

$$
\sum_{\alpha^{\prime}+\alpha^{\prime \prime}=\alpha}\left(\begin{array}{c}
\alpha \\
\alpha^{\prime}
\end{array}\right) \Gamma_{s}\left(\left|\alpha^{\prime}\right|+p+1\right) \Gamma_{s}\left(\left|\alpha^{\prime \prime}\right|+q\right) \leq \Gamma_{s}(|\alpha|+p+q)
$$

for $p \geq 0$ and $q \geq 1, p, q \in \mathbb{N}$,

(ii)

$$
\sum_{\alpha^{\prime}+\alpha^{\prime \prime}=\alpha}\left(\begin{array}{c}
\alpha \\
\alpha^{\prime}
\end{array}\right) \Gamma_{s}\left(\left|\alpha^{\prime}\right|+p\right) \Gamma_{s}\left(\left|\alpha^{\prime \prime}\right|\right) \leq \Gamma_{s}(|\alpha|+p)
$$

for $p \geq 0$. 


\section{LEMMA A.2}

Assume that

$$
\begin{gathered}
\left|\partial_{x}^{\alpha} \phi_{j}(x)\right| \leq C_{j} A_{1}^{\alpha} \Gamma_{s}(|\alpha|-1), \quad|\alpha| \geq 1, j=1,2, \ldots, N \\
\left|\partial_{z}^{\alpha} u(z)\right| \leq C A_{2}^{\alpha} \Gamma_{s}(|\alpha|-1), \quad|\alpha| \geq 1,
\end{gathered}
$$

where $C_{j}=C_{j}(x), A_{1}(x)=\left(A_{11}(x), \ldots, A_{1 n}(x)\right), A_{2}(z)=\left(A_{21}(z), \ldots, A_{2 N}(z)\right)$, and $u(z)=u\left(z_{1}, \ldots, z_{N}\right)$. Let

$$
d(x, z)=\sum_{j=1}^{N} C_{j}(x) A_{2 j}(z) .
$$

Then we have, with $\phi(x)=\left(\phi_{1}(x), \ldots, \phi_{n}(x)\right)$,

$$
\left|\partial_{x}^{\gamma} u(\phi(x))\right| \leq C d(x, \phi(x))(1+d(x, \phi(x)))^{|\gamma|-1} A_{1}^{\gamma} \Gamma_{s}(|\gamma|-1), \quad|\gamma| \geq 1
$$

\section{LEMMA A.3}

Assume that

$$
\begin{array}{r}
\left|\partial_{x}^{\alpha} \phi_{j}(x)\right| \leq C_{j} A_{1}^{\alpha} \Gamma_{s}(|\alpha|-1), \quad|\alpha| \geq 1, j=1, \ldots, N, \\
\left|\left[\partial_{x}^{\alpha} \partial_{z}^{\gamma} F(x, z)\right]_{z=\phi(x)}\right| \leq C A_{2}^{\alpha} B_{2}^{\gamma} \Gamma_{s}(|\alpha+\gamma|-1), \quad|\alpha+\gamma| \geq 1,
\end{array}
$$

for $|\alpha+\gamma| \leq k,|\alpha| \leq k$, where $C_{j}=C_{j}(x), A_{1}(x)=\left(A_{11}(x), \ldots, A_{1 m}(x)\right)$, $A_{1 j}(x)>0, A_{2}(x)=\left(A_{21}(x), \ldots, A_{2 m}(x)\right)$, and $B_{2}=\left(B_{21}(x), \ldots, B_{2 N}(x)\right)$. Assume that

$$
\sum_{j=1}^{N} C_{j} B_{2 j}+\sum_{j=1}^{m} A_{2 j} A_{1 j}^{-1} \leq 1
$$

then we have

$$
\left|\partial_{x}^{\mu} F_{(\alpha)}^{(\gamma)}(x, \phi(x))\right| \leq C A_{2}^{\alpha} B^{\gamma} A_{1}^{\mu} \Gamma_{s}(|\alpha+\mu+\gamma|-1)
$$

for $|\alpha+\gamma+\mu| \leq k$, where $F_{(\alpha)}^{(\gamma)}(x, z)=\partial_{x}^{\alpha} \partial_{z}^{\gamma} F(x, z)$.

\section{COROLLARY A.2}

Assume that

$$
\begin{gathered}
\left|\partial_{x}^{\alpha} \phi_{j}(x)\right| \leq C_{j} A_{1}^{\alpha} \Gamma_{s}(|\alpha|-1), \quad|\alpha| \geq 1, j=1, \ldots, N \\
\left|\left[\partial_{x}^{\alpha} \partial_{y}^{\beta} \partial_{z}^{\gamma} F(x, y, z)\right]_{z=\phi}\right| \leq C A_{2}^{\alpha} B_{2}^{\beta} D_{2}^{\gamma} \Gamma_{s}(|\alpha+\beta+\gamma|-1), \quad|\alpha+\beta+\gamma| \geq 1,
\end{gathered}
$$

for $|\alpha+\beta+\gamma| \leq k,|\alpha| \leq k$, where $C_{j}=C_{j}(x), A_{1}(x)=\left(A_{11}(x), \ldots, A_{1 m}(x)\right)$, $A_{1 j}(x)>0, A_{2}(x)=\left(A_{21}(x), \ldots, A_{2 m}(x)\right), B_{2}(x, y)=\left(B_{21}(x, y), \ldots, B_{2 \ell}(x, y)\right)$, and $D_{2}(x, y)=\left(D_{21}(x, y), \ldots, D_{2 N}(x, y)\right)$. Assume that

$$
\sum_{j=1}^{N} C_{j} D_{2 j}+\sum_{j=1}^{m} A_{2 j} A_{1 j}^{-1} \leq 1 ;
$$

then we have

$$
\left|\partial_{x}^{\mu} \partial_{y}^{\nu} F(x, y, \phi(x))\right| \leq C A_{1}^{\mu} B_{2}^{\nu} \Gamma_{s}(|\mu+\nu|-1)
$$

for $|\mu+\nu| \leq k$ 


\section{A.3 Estimates of some special symbols}

\section{LEMMA A.4}

Assume that $\phi \in S_{(s)}(k(\xi, \mu), g)$. Let $\omega_{\beta}^{\alpha}=e^{-\phi} \partial_{x}^{\beta} \partial_{\xi}^{\alpha} e^{\phi}$. Then we have, with some constants $A_{i}>0$,

$$
\begin{aligned}
\left|\partial_{x}^{\nu} \partial_{\xi}^{\mu} \omega_{\beta}^{\alpha}\right| \leq & C A_{1}^{|\nu+\mu|} A_{2}^{|\alpha+\beta|} \delta^{\beta+\nu} \rho^{-\alpha-\mu} \\
& \times \sum_{j=0}^{|\alpha+\beta|} k(\xi)^{|\alpha+\beta|-j}(|\mu+\nu|+j) ! .
\end{aligned}
$$

\section{COROLLARY A.3}

We have

$$
\begin{aligned}
\left|\partial_{x}^{\beta} \partial_{\xi}^{\alpha} e^{\phi}\right| & \leq C e^{\phi} A^{|\alpha+\beta|} \delta^{\beta} \rho^{-\alpha} \sum_{j=0}^{|\alpha+\beta|} k(\xi)^{|\alpha+\beta|-j} j !^{s} \\
& \leq C A_{1}^{|\alpha+\beta|}|\alpha+\beta| !^{s} \delta^{\beta} \rho^{-\alpha} e^{\phi} e^{k(\xi)^{1 / s}}
\end{aligned}
$$

\section{LEMMA A.5}

Assume that $\phi(x)$ verifies

$$
\left|\partial_{x}^{\alpha} \phi(x)\right| \leq C_{1} A_{1}(x)^{\alpha}|\alpha| !^{s}, \quad x \in \mathbb{R}^{n} .
$$

For given $m, \ell \in \mathbb{N}$, let us set

$$
w(x)=\left(\phi(x)^{2 m}+B^{-2}\right)^{1 / \ell} .
$$

Then we have

$$
\left|\partial_{x}^{\alpha} w(x)\right| \leq C C_{2}^{|\alpha|} w(x) w(x)^{-\ell|\alpha| / 2 m} A_{1}(x)^{\alpha}|\alpha| !^{s}
$$

for $x \in \mathbb{R}$.

\section{COROLLARY A.4}

Assume that

$$
\left|\partial_{x}^{\beta} \partial_{\xi}^{\alpha} \phi(x, \xi)\right| \leq C A^{|\alpha+\beta|}|\alpha+\beta| !^{s}\langle\xi\rangle_{\mu}^{-|\alpha|} .
$$

Then we have, with $w(x, \xi)=\left(\phi(x, \xi)^{2 m}+\langle\xi\rangle_{\mu}^{-p}\right)^{1 / \ell}$,

$$
\left|\partial_{x}^{\beta} \partial_{\xi}^{\alpha} w(x, \xi)\right| \leq C_{1} A_{1}^{|\alpha+\beta|}|\alpha+\beta| !^{s} w(x, \xi) w(x, \xi)^{-\ell|\alpha+\beta| / 2 m}\langle\xi\rangle_{\mu}^{-|\alpha|},
$$

that is, $w \in S_{(s)}\left(w, w^{-\ell / m}\left(|d x|^{2}+\langle\xi\rangle_{\mu}^{-2}|d \xi|^{2}\right)\right)$. In particular, $w \in S_{(s)}(w$, $\left.\langle\xi\rangle_{\mu}^{p / m}\left(|d x|^{2}+\langle\xi\rangle_{\mu}^{-2}|d \xi|^{2}\right)\right)$.

\section{COROLLARY A.5}

We have

$$
w^{-1} \in S_{(s)}\left(w^{-1},\langle\xi\rangle_{\mu}^{p / m}|d x|^{2}+\langle\xi\rangle_{\mu}^{-2+p / m}|d \xi|^{2}\right) .
$$


Proof

Since $\left|(d / d x)^{k} x^{-1}\right|=k !|x|^{-k-1}$ the assertion follows from Lemma A.2.

Let $\psi(s)$ satisfy

$$
\left|\psi^{(k)}(s)\right| \leq C A^{k} k !^{s}, \quad s \in \mathbb{R}
$$

and consider

$$
\phi=\log \left(\psi\left(x_{1}\right)+i w\right)-\log \left(\psi\left(x_{1}\right)-i w\right) .
$$

We set

$$
r(x, \xi)=\sqrt{\psi\left(x_{1}\right)^{2}+w(x, \xi)^{2}} .
$$

\section{LEMMA A.6}

Let

$$
g^{*}=\left(r(x, \xi)^{-1}+w^{-\ell / 2 m}\right)^{2} d x_{1}^{2}+\sum_{j=2}^{n} w^{-\ell / m} d x_{j}^{2}+w^{-\ell / m}\langle\xi\rangle_{\mu}^{-2}|d \xi|^{2} .
$$

Then we have $\phi(x, \xi) \in S_{(s)}\left(1, g^{*}\right)$ and $r(x, \xi) \in S_{(s)}\left(r, g^{*}\right)$.

Proof

For $|\alpha+\beta|=1$ we have

$$
\partial_{x}^{\beta} \partial_{\xi}^{\alpha} \phi=-2 i r(x, \xi)^{-2}\left[w(x, \xi) \partial_{x}^{\beta} \partial_{\xi}^{\alpha} \psi\left(x_{1}\right)-\psi\left(x_{1}\right) \partial_{x}^{\beta} \partial_{\xi}^{\alpha} w\right] .
$$

Since it is clear that $\psi\left(x_{1}\right) \in S_{(s)}\left(r, g^{*}\right)$ and $w \in S_{(s)}\left(r, g^{*}\right)$, to prove the assertion it is enough to prove $r(x, \xi)^{-2} \in S_{(s)}\left(r^{-2}, g^{*}\right)$. Noting that

$$
\left|(d / d x)^{k} x^{1 / 2}\right| \leq C A^{k} k ! x^{1 / 2} x^{-k}, \quad x>0,
$$

and $r^{2}=\psi\left(x_{1}\right)^{2}+w^{2}(x, \xi) \in S_{(s)}\left(r^{2}, g^{*}\right)$, applying Lemma A.2 we get $r \in$ $S_{(s)}\left(r, g^{*}\right)$. Again applying Lemma A.2 we conclude that $r^{-1} \in S_{(s)}\left(r^{-1}, g^{*}\right)$, which proves the assertion.

\section{A.4 Implicit functions $\Xi(x, y, \zeta)$}

Let $\phi(x, \xi) \in S_{(s)}(\bar{k}(\xi, \mu), g)$ be real valued, and assume that $\partial_{x_{j}} \phi(x, \xi) \in$ $S_{(s)}\left(\bar{k} \Delta_{j}, g\right)$, where $\bar{k}$ is assumed to satisfy (A.1) and $\Delta_{j} \in S_{(s)}\left(\Delta_{j}, g\right)$. Set

$$
F(x, y, \zeta)=\int_{0}^{1} \nabla_{x} \phi(y+\theta(x-y), \zeta) d \theta, \quad \zeta=\xi+i \eta,
$$

where $\nabla_{x} \phi(x, \zeta)$ is an almost analytic extension of $\nabla_{x} \phi(x, \xi)$ with $k=\bar{k}\langle\xi\rangle_{\mu}^{\epsilon^{\prime}}$ and small $0<\epsilon^{\prime}<\epsilon$.

PROPOSITION A.3

There is a $C^{\infty}$-function $\Xi(x, y, \zeta)=\zeta+G(x, y, \zeta)$ defined for $(x, \xi, \eta ; y) \in$ $E^{0}\left(\bar{k}\langle\xi)_{\mu}^{\epsilon^{\prime \prime}}\right) \times \mathbb{R}^{n}\left(0<\epsilon^{\prime \prime}<\epsilon^{\prime}\right)$ such that

$$
\Xi(x, y, \zeta)=i F(x, y, \Xi(x, y, \zeta))+\zeta,
$$


where $G_{j}(x, y, \zeta)$ satisfies, for $(x, \xi, \eta ; y) \in E^{0}\left(\bar{k}\langle\xi\rangle_{\mu}^{\epsilon^{\prime \prime}}\right) \times \mathbb{R}^{n}$,

$$
\left|\partial_{x, y}^{\beta} \partial_{\xi, \eta}^{\alpha} G_{j}(x, y, \zeta)\right| \leq C A^{|\alpha+\beta|}|\alpha+\beta|^{!} \bar{k}\langle\xi\rangle_{\mu}^{a_{j}}\langle\xi\rangle_{\mu}^{a \beta}\langle\xi\rangle_{\mu}^{-b \alpha} .
$$

Moreover, we have

$$
\left[\partial_{x, y}^{\beta} \partial_{\xi, \eta}^{\alpha} G_{j}(x, y, \zeta)\right]_{y=x} \in S_{(s)}\left(\bar{k} \Delta_{j} \delta^{\beta} \rho^{-\alpha}, g \mid E^{0}\left(\bar{k}\langle\xi\rangle_{\mu}^{\epsilon^{\prime \prime}}\right)\right), \quad|\alpha+\beta| \geq 1 .
$$

We have also

$$
\left|\partial_{x, y}^{\beta} \partial_{\xi, \eta}^{\alpha} \bar{\partial}_{\zeta_{j}} \Xi(x, y, \zeta)\right| \leq C A^{|\alpha+\beta|}|\alpha+\beta| !^{s} h e^{-c\left(h \bar{k}\langle\xi\rangle_{\mu}^{\epsilon^{\prime \prime}}\right)^{-1 /(s-1)}}\langle\xi\rangle_{\mu}^{a \beta}\langle\xi\rangle_{\mu}^{-b \alpha}
$$

with some $c>0$ for $(x, \xi, \eta ; y) \in E^{0}\left(\bar{k}\langle\xi\rangle_{\mu}^{\epsilon^{\prime \prime}}\right) \times \mathbb{R}^{n}$.

\section{B}

In this section we use

$$
\bar{g}(y, \eta)=\sum_{j=1}^{n}\langle\xi\rangle_{\mu}^{2 \delta} y_{j}^{2}+\langle\xi\rangle_{\mu}^{-2 \rho} \eta_{j}^{2}, \quad g_{\rho}(y, \eta)=\sum_{j=1}^{n} y_{j}^{2}+\langle\xi\rangle_{\mu}^{-2 \rho} \eta_{j}^{2},
$$

where $0<\delta<\rho \leq 1$. Let $p(x, \xi) \in S_{(s)}\left(\langle\mu \xi\rangle^{m}, \bar{g}\right)$, and let $\phi \in S_{(s)}\left(\langle\mu \xi\rangle^{\kappa^{\prime}}, \bar{g}\right)$ which is real valued such that

$$
\nabla_{\xi} \phi \in S_{(s)}\left(\langle\mu \xi\rangle^{\kappa}\langle\xi\rangle_{\mu}^{-\rho}, \bar{g}\right), \quad \nabla_{x} \phi(x, \xi) \in S_{(s)}\left(\langle\mu \xi\rangle^{\kappa}\langle\xi\rangle_{\mu}^{\delta}, \bar{g}\right) .
$$

We assume that $\left(\kappa^{\prime} \geq \kappa, s>1\right)$

$$
(s-1) \kappa^{\prime},(s-1)(1-\rho+\kappa)<\rho-\delta-\kappa, \quad s \kappa^{\prime}<1-\delta .
$$

Recall

$$
\mathrm{Op}^{t}\left(e^{\phi} p\right) u=(2 \pi)^{-n} \int e^{i(x-y) \xi+\phi(t y+(1-t) x, \xi)} p(t y+(1-t) x, \xi) u(y) d y d \xi,
$$

where $0 \leq t \leq 1$. Let us put

$$
\Phi(x, \xi, \eta)=\int_{0}^{1} \nabla_{\xi} \phi(x, \xi+\theta \eta) d \theta
$$

so that $\phi(x, \xi+\eta)-\phi(x, \xi)=\eta \Phi(x, \xi, \eta)$. Then we have the following.

\section{PROPOSITION B.1}

Assume (B.1). Then we have

$$
\mathrm{Op}^{0}\left(e^{\phi}\right) \mathrm{Op}^{0}(p)=\mathrm{Op}^{0}\left(e^{\phi} q\right)+\mathrm{Op}^{0}(r)
$$

where $q \in S_{(s)}\left(\left\langle\mu \xi^{\prime}\right\rangle^{m}, \bar{g}\right)$ and $r \in S_{(s d)}\left(e^{-c\left(\mu^{-1}\langle\mu \xi\rangle\right)^{(1-\delta) / s}}, g_{\rho}\right)$ with $d=(1+\rho-$ $\delta) /(1-\delta)$, and one can write

$$
q(x, \xi)=\left.\sum_{|\beta|<N} \frac{1}{\beta !} \partial_{\eta}^{\beta} p_{(\beta)}(x-i \Phi(x, \xi, \eta), \xi)\right|_{\eta=0}+q_{N}(x, \xi)
$$

with $q_{N} \in \mu^{(\rho-\delta) N} S_{(s)}\left(\langle\mu \xi\rangle^{m-(\rho-\delta) N}, \bar{g}\right)$, where $p_{(\beta)}(x+i y, \xi)$ is the almost analytic extension of $(-i)^{|\beta|} \partial_{x}^{\beta} p(x, \xi)$ given by Proposition A.1. 
Consider $\mathrm{Op}^{0}\left(e^{\phi} q\right) \mathrm{Op}^{1}\left(e^{-\phi}\right)$, where $q \in S_{(s)}\left(\langle\mu \xi\rangle^{m}, \bar{g}\right)$. Let $\Xi(x, y, \xi)$ be the solution to

$$
\Xi-i \int_{0}^{1} \nabla_{x} \phi(x+\theta(y-x), \Xi) d \theta=\xi
$$

which is given by Proposition A.3. Then we have the following.

\section{PROPOSITION B.2}

Assume (B.1). Then one can write

$$
\mathrm{Op}^{0}\left(e^{\phi} q\right) \mathrm{Op}^{1}\left(e^{-\phi}\right)=\mathrm{Op}^{0}(p)+\mathrm{Op}^{0}(r),
$$

where $p(x, \xi) \in S_{(s)}\left(\langle\mu \xi\rangle^{m}, \bar{g}\right)$ and $r(x, \xi) \in S_{(s d)}\left(e^{-c\left(\mu^{-1}\langle\mu \xi\rangle\right)^{(1-\delta) / s}}, g_{\rho}\right)$, and we can write

$$
p(x, \xi)=\sum_{|\alpha|<N} \frac{1}{\alpha !} \partial_{\xi}^{\alpha} D_{y}^{\alpha}[J(x, y, \xi) q(x, \Xi(x, y, \xi))]_{y=x}+R_{N}(x, \xi)
$$

with $R_{N}(x, \xi) \in \mu^{(\rho-\delta) N} S_{(s)}\left(\langle\mu \xi\rangle^{m-(\rho-\delta) N}, \bar{g}\right)$, where

$$
J(x, y, \xi)=\operatorname{det}\left[\frac{\partial \Xi(x, y, \xi)}{\partial \xi}\right] .
$$

PROPOSITION B.3

Let $s>1$. Assume that

$$
p(x, \xi) \in S_{(s)}\left(e^{c \mu^{-a}\langle\mu \xi\rangle^{\kappa}}\langle\mu \xi\rangle^{m_{1}}, \bar{g}\right), \quad q(x, \xi) \in S_{(s)}\left(e^{c^{\prime} \mu^{-a}\langle\mu \xi\rangle^{\kappa}}\langle\mu \xi\rangle^{m_{2}}, \bar{g}\right)
$$

with $c+c^{\prime}<0$ and some $a>0$. Then one can write

$$
\mathrm{Op}^{0}(p) \mathrm{Op}^{1}(q)=\mathrm{Op}^{0}\left(r_{1}\right)+\mathrm{Op}^{0}\left(r_{2}\right)
$$

with $r_{1}(x, \xi) \in S_{(s)}\left(\langle\mu \xi\rangle^{m_{1}+m_{2}} e^{-c_{1} \mu^{-a}\langle\mu \xi\rangle^{\kappa}}, \bar{g}\right), r_{2}(x, \xi) \in S_{(s d)}\left(e^{-c_{2}\left(\mu^{-1}\langle\mu \xi\rangle\right)^{(1-\delta) / s}}\right.$, $\left.g_{\rho}\right)$ with some $c_{i}>0$. In particular, we have $\mathrm{Op}^{0}(p) \mathrm{Op}^{1}(q) \in \mu^{k} S_{(s d)}\left(\langle\mu \xi\rangle^{-k}, \bar{g}\right)$ for any $k \in \mathbb{N}$.

\section{COROLLARY B. 1}

Assume (B.1) and $p(x, \xi) \in S_{(s)}\left(\langle\mu \xi\rangle^{m}, \bar{g}\right)$. Then we have

$$
\mathrm{Op}^{0}\left(e^{-\phi}\right) \mathrm{Op}^{0}(p) \mathrm{Op}^{1}\left(e^{\phi}\right)=\mathrm{Op}^{0}(\tilde{p})+\mathrm{Op}^{0}(r),
$$

where $\tilde{p}(x, \xi) \in S_{(s)}\left(\langle\mu \xi\rangle^{m}, \bar{g}\right)$ and $r(x, \xi) \in \mu^{k} S_{\left(s d^{2}\right)}\left(\langle\mu \xi\rangle^{-k}, \bar{g}\right)$ for any $k \in \mathbb{N}$.

Acknowledgments. We are very grateful to the referees for a careful reading of the manuscript and for pointing out a lack of arguments in the proof of Theorem 1.1, which now have been added.

\section{References}

[1] R. Beals, Characterization of pseudodifferential operators and applications, Duke Math. J. 44 (1977), 45-57. 
[2] E. Bernardi, A. Bove, and C. Parenti, Geometric results for a class of hyperbolic operators with double characteristics, II, J. Funct. Anal. 116 (1993), 62-82.

[3] E. Bernardi and T. Nishitani, On the Cauchy problem for non-effectively hyperbolic operators, the Gevrey 5 well-posedness, J. Anal. Math. 105 (2008), $197-240$.

[4] M. D. Bronšteĭn, The Cauchy problem for hyperbolic operators with characteristics of variable multiplicity (in Russian), Trudy Moscov. Mat. Obshch. 41 (1980), 83-99.

[5] J. J. Duistermaat, Fourier Integral Operators, reprint of the 1996 original, Birkhäuser/Springer, New York, 2011.

[6] L. Hörmander, The Cauchy problem for differential equations with double characteristics, J. Anal. Math. 32 (1977), 118-196.

[7] _ "Quadratic hyperbolic operators" in Microlocal Analysis and Applications (Montecatini Terme, Italy, 1989), Lecture Notes in Math. 1495, Springer, Berlin, 1991, 118-160.

[8] - The Analysis of Linear Partial Differential Operators, III, reprint of the 1994 ed., Classics Math., Springer, Berlin, 2007.

[9] V. Ja. Ivrii, Correctness of the Cauchy problem in Gevrey classes for non-strictly hyperbolic operators, Math. USSR Sbornik 25 (1975), 365-387.

[10] V. Ja. Ivrii and V. M. Petkov, Necessary conditions for the correctness of the Cauchy problem for non-strictly hyperbolic equations (in Russian), Uspehi Mat. Nauk. 29 (1974), no. 5, 3-70.

[11] K. Kajitani and T. Nishitani, The Hyperbolic Cauchy Problem, Lecture Notes in Math. 1505, Springer, Berlin, 1991.

[12] H. Kumano-go, Pseudo-Differential Operators, MIT Press, Cambridge, Mass., 1982.

[13] T. Nishitani, Local energy integrals for effectively hyperbolic operators, I, J. Math. Kyoto Univ. 24 (1984), 623-658; II, 659-666.

[14] _ "Microlocal energy estimates for hyperbolic operators with double characteristics" in Hyperbolic Equations and Related Topics (Katata/Kyoto, 1984), Academic Press, Boston, 1986, 235-255.

[15] Non effectively hyperbolic operators, Hamilton map and bicharacteristics, J. Math. Kyoto Univ. 44 (2004), 55-98.

[16] - "On Gevrey well-posedness of the Cauchy problem for some noneffectively hyperbolic operators" in Advances in Phase Space Analysis of Partial Differential Equations: In Honor of Ferruccio Colombini's 60th Birthday, Nonlinear Differential Equations Appl. 78, Birkhäuser, Boston, 2009, 217-233.

[17] T. Nishitani and M. Tamura, A class of Fourier integral operators with complex phase related to the Gevrey classes, J. Pseudo-Differ. Oper. Appl. 1 (2010), 255-292. 
Bernardi: Dipartimento di Matematica Per Le Scienze Economiche e Sociali, Università di Bologna, Viale Filopanti 5, 40126 Bologna, Italy; enrico.bernardi@gmail.com

Nishitani: Department of Mathematics, Osaka University, Machikaneyama 1-1, Toyonaka, 560-0043, Osaka, Japan; nishitani@math.sci.osaka-u.ac.jp 